\title{
WAVE LENGTHS LONGER THAN 5500 A IN THE ARC SPECTRA OF SEVEN ELEMENTS
}

\author{
By C. C. Kiess and W. F. Meggers
}

CONTENTS

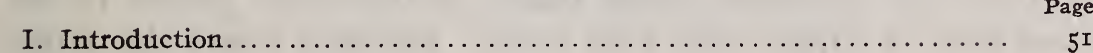

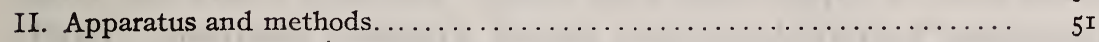

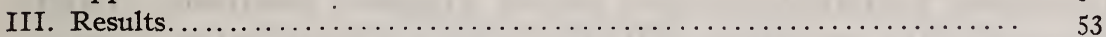

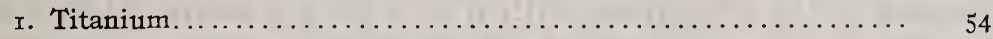

2. Vanadium ................................... ${ }_{56}^{6}$

3. Chromium....................................... $5_{58}^{8}$

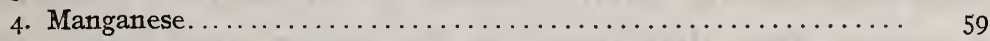

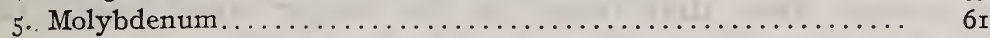

6. Tungsten $\ldots \ldots \ldots \ldots \ldots \ldots \ldots \ldots \ldots \ldots \ldots \ldots \ldots \ldots \ldots \ldots \ldots \ldots \ldots, 6_{4}$

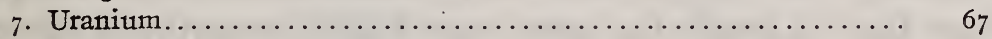

8. Faint lines common to several spectra................. $7^{2}$

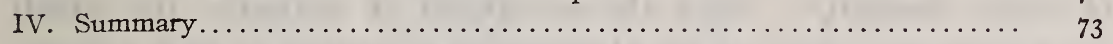

\section{INTRODUCTION}

The wave lengths in the arc spectra of titanium, vanadium, chromium, manganese, molybdenum, tungsten, and uranium presented in this paper form a continuation of the program of standard wave-length determination and spectroscopic analysis inaugurated at the Bureau of Standards several years ago. This program has as one of its objects the mapping of the red and infra-red spectra of the chemical elements as far out into the region of longer wave lengths as modern photographic methods will permit. The values given below are submitted, therefore, not only as a description of the red and infra-red regions of the various spectra, but also to serve as starting values for the more precise determinations of interferometry. Similar results for I 5 other elements have thus far been published. ${ }^{1}$ Work on others is in various stages of progress, and it is hoped that this phase of the program may be completed within a few years.

\section{APPARATUS AND METHODS}

In the present work no essential departure was made from the methods of observing described in the papers referred to above. All the plates were exposed in the first order of the concave grating with the exception of two, for which the second order was used in photographing the region from $6500-7500 \mathrm{~A}$ 
for chromium, tungsten, and molybdenum. The plates were measured on a large engine constructed by Gærtner, which is illustrated in Fig. I. Its smallest scale division is $0.001 \mathrm{~mm}$, which corresponds to o.oro $\mathrm{A}$ in photographs of the first-order spectrum.

For tungsten and molybdenum, rods served as electrodes; for the other elements, small pieces of the metal placed in the cored ends of copper rods were used. We have found this latter procedure preferable to the use of graphite electrodes, partly because of the steadiness with which the arc burns when a copper bead from the upper electrode is fused with the metal in the lower electrode and partly because of the relatively small number of copper lines that occur in the longer wave-length regions. Some of these copper lines may in rare instances be inseparable from lines of nearly the same wave length occurring in the spectra of other elements. With the exception of uranium, for which the nitrate was used, all the elements were burned in their uncombined form.

The photographs were made on Seed 27 and Seed 30 plates sensitized to the yellow and red regions of the spectrum with pinacyanol, dicyanin, and dicyanin A. The methods of using these dyes have been frequently described. In the course of other work carried on by the spectroscopic laboratory of the Bureau of Standards it became part of the routine work to test photographic dyes made by various laboratories to replace the German product, which was cut off by the war. Many of the plates used in the present study were sensitized with these new dyes, and it is a pleasure to testify that the pinacyanols produced in the United States are equal to their predecessors in every respect.

The arc spectrum of iron serveu as a comparison spectrum, and its wave lengths determined by interference methods ${ }^{2}$ were used in the reductions. The duration of exposures increased from about five minutes for the short waves to about five hours for the longest. In the case of long exposures, to eliminate temperature shifts the plate was exposed to the iron spectrum both before and after. Since, however, the room containing the spectrograph remains at practically constant temperature for long intervals of time, it is felt that the wave lengths presented below are free from error of this kind. 


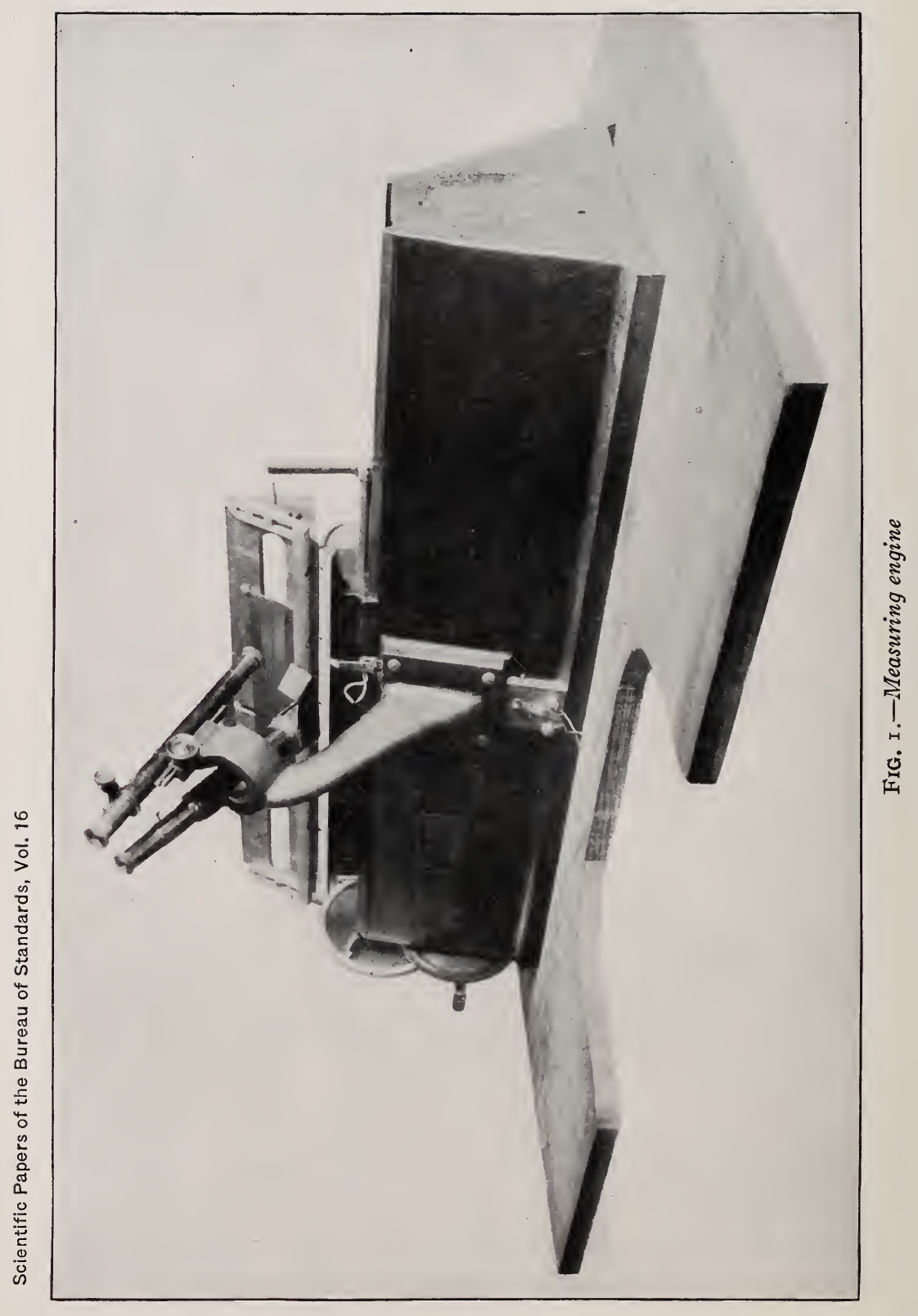




\section{RESULTS}

Each wave length given in the following tables is, in general, the mean of from two to eight determinations. Each plate in the first order covers 2000 angstrom units, and three or more photographs were made of spectral regions which overlap each other by half this interval. Lines observed only once or twice were recorded by longer exposure than the average for a particular region.

Following each wave length are notes giving the intensity and character of the line. The intensities range from $\mathrm{I}$ for the faintest lines measurable to Io for the strongest.

The letters have the following significance:

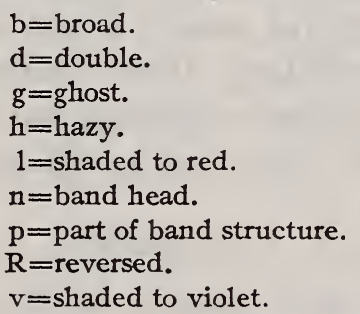

The last column of the tables indicates the probable errors of the measurement of each wave length, the letters having the significance:

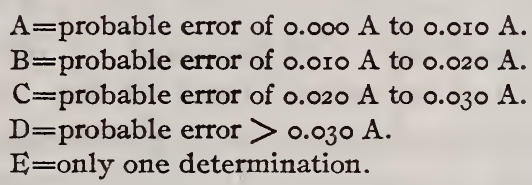

Lines with probable errors $A$ and $B$ were measured three times or more.

It is hoped that these tables are free from the wave lengths of impurities, but although all the chemicals which were burned in the arc were the purest obtainable, yet it was found that none were spectroscopically pure. A number of faint lines were found to be common to the spectra of two or more elements. These are caused, no doubt, by some common impurities which, as yet, havie not been identified. All such lines are given in Table 8, following the tables containing the wave lengths of the elements.

During the work on the spectrum of chromium it was found that a number of the infra-red lines were repetitions of the three strong lines at wave lengths 7355,7400 , and $7462 \mathrm{~A}$. These 
spurious lines are of the type first discovered by Lyman, ${ }^{3}$ although the factors relating the false to the parent lines are not the same as those given by him. In all, seven factors giving false lines of apparent wave length greater than the parent line have been detected. These factors have been used to eliminate all false lines from the present tables. Unfortunately, our tables for iron, cobalt, and nickel, which were published at a time when it seemed that war activities would preclude further spectroscopic work in this laboratory for an indefinite period, contain many of these false lines.

The seriousness of this weakness inherent in "work done with grating spectrographs is apparent and suggests that all gratings should be investigated for their "instrumental constants." To determine more exactly the nature of the false spectra produced by gratings, and particularly their relation to the method of ruling, a number of concave gratings have been.borrowed and are now being studied. The results will be presented in a later paper.

\section{TITANIUM}

Approximately 200 wave lengths in the arc spectrum of titanium have been measured between the limits $5500 \mathrm{~A}$ and $9800 \mathrm{~A}$. About one-tenth of the wave lengths presented are band heads, the bands, no doubt, having their origin in the oxide of titanium. Hundreds of lines belonging to bands were not resolved and were therefore not measured. The presence of bands and the relatively small number of strong lines in the red render the arc spectrum of this metal undesirable as a reference spectrum for the red regions. Kilby ${ }^{4}$ has measured the wave lengths of the spectrum in international units, but his measurements extend only to 626I A.

Among the wave lengths presented below (Table I) are several pairs with constant frequency differences. Two of these pairs are of the type found by Paulson. ${ }^{5}$ The data are as follows:

\begin{tabular}{|c|c|c|c|c|}
\hline$\lambda$ & & $\nu$ & $\Delta \nu$ & \\
\hline 5762.25 & $2 \mathrm{~h}$ & 17354.33 & 35.38 & \\
5774.02 & $2 \mathrm{~h}$ & 17318.95 & & \\
5953.18 & 3 & 16797.74 & 35.56 & 71.31 \\
5965.81 & 3 & 16762.18 & 35.75 & \\
5978.56 & 3 & -16726.43 & & \\
7978.87 & 2 & 12533.10 & 71.78 & \\
8024.83 & 2 & 12461.32 & & \\
\hline
\end{tabular}


TABLE 1.-Arc Spectrum of Titanium

\begin{tabular}{|c|c|c|c|c|c|c|c|c|}
\hline$\lambda$ I. A. & Notes & $\begin{array}{c}\text { Probable } \\
\text { error }\end{array}$ & $\lambda$ I. A. & Notes & $\begin{array}{c}\text { Probable } \\
\text { error }\end{array}$ & $\lambda$ I. A. & Notes & $\begin{array}{c}\text { Probable } \\
\text { error }\end{array}$ \\
\hline $\begin{array}{l}5503.88 \\
5512.49 \\
5514.46 \\
5565.48 \\
5597.92\end{array}$ & $\begin{array}{l}2 \\
4 \\
5, \mathrm{~d} \\
2 \\
2, \mathrm{n}\end{array}$ & $\begin{array}{l}\mathbf{B} \\
\mathbf{C} \\
\mathbf{C} \\
\mathbf{B} \\
\mathbf{C}\end{array}$ & $\begin{array}{l}6295.32 \\
6303.76 \\
6305.65 \\
6312.23 \\
6316.42\end{array}$ & $\begin{array}{l}1 \\
2 \\
1 \\
1 \\
1\end{array}$ & $\begin{array}{l}\mathbf{E} \\
\mathbf{A} \\
\mathbf{E} \\
\mathbf{B} \\
\mathbf{E}\end{array}$ & $\begin{array}{l}7978.87 \\
7996.49 \\
8003.55 \\
8024.83 \\
8034.99\end{array}$ & $\begin{array}{l}4 \\
3 \\
1 \\
2 \\
1\end{array}$ & $\begin{array}{l}\mathbf{A} \\
\mathbf{A} \\
\mathbf{E} \\
\mathbf{A} \\
\mathbf{E}\end{array}$ \\
\hline $\begin{array}{l}5629.39 \\
5644.21 \\
5648.63 \\
5662.12 \\
5675.48\end{array}$ & $\begin{array}{l}2, \mathrm{n} ? \\
3 \\
2 \\
2, \mathrm{~d} ? \\
2\end{array}$ & $\begin{array}{l}\mathbf{B} \\
\mathbf{C} \\
\mathbf{B} \\
\mathbf{B} \\
\mathbf{A}\end{array}$ & $\begin{array}{l}6336.10 \\
6366.38 \\
6446.27 \\
6454.22 \\
6456.06\end{array}$ & $\begin{array}{l}\frac{1}{2}, \mathrm{Ni} ? \\
2 \\
2 \\
2, \mathrm{O} ?\end{array}$ & $\begin{array}{l}\mathbf{B} \\
\mathbf{B} \\
\mathbf{B} \\
\mathbf{B} \\
\mathbf{B}\end{array}$ & $\begin{array}{l}8064.09 \\
8068.21 \\
8069.73 \\
8127.91 \\
8131.40\end{array}$ & $\begin{array}{l}2, p ? \\
2 \\
1 \\
1 \\
1\end{array}$ & $\begin{array}{l}\mathbf{E} \\
\mathbf{B} \\
\mathbf{E} \\
\mathbf{E} \\
\mathbf{E}\end{array}$ \\
\hline $\begin{array}{l}5679.97 \\
5689.51 \\
5702.67 \\
5708.27 \\
5713.93\end{array}$ & $\begin{array}{l}1 \\
2 \\
1 \\
1 \\
1\end{array}$ & $\begin{array}{l}\mathbf{B} \\
\mathbf{A} \\
\mathbf{B} \\
\mathbf{A} \\
\mathbf{B}\end{array}$ & $\begin{array}{l}6464.02 \\
6465.56 \\
647.18 \\
6497.71 \\
6508.15\end{array}$ & $\begin{array}{l}1 \\
2 \\
1 \\
1 \\
1\end{array}$ & $\begin{array}{l}\mathbf{E} \\
\mathbf{E} \\
\mathbf{E} \\
\mathbf{E} \\
\mathbf{E}\end{array}$ & $\begin{array}{l}8180.45 \\
8207.32 \\
8241.49 \\
8267.62 \\
8289.07\end{array}$ & $\begin{array}{l}1, \mathrm{n} ? \\
1, \mathrm{n} ? \\
1 \\
1, \mathrm{n} ?\end{array}$ & $\begin{array}{l}\mathbf{E} \\
\mathbf{E} \\
\mathbf{E} \\
\mathbf{E} \\
\mathbf{E}\end{array}$ \\
\hline $\begin{array}{l}5716.48 \\
5720.52 \\
5739.46 \\
5739.96 \\
5741.28\end{array}$ & $\begin{array}{l}1 \\
1 \\
2 \\
1 \\
1\end{array}$ & $\begin{array}{l}\mathbf{A} \\
\mathbf{B} \\
\mathbf{A} \\
\mathbf{B} \\
\mathbf{B}\end{array}$ & $\begin{array}{l}6546.26 \\
6554.22 \\
6556.09 \\
6575.19 \\
6599.13\end{array}$ & $\begin{array}{l}4 \\
3,1 \\
3 \\
1 \\
3\end{array}$ & $\begin{array}{l}\mathbf{B} \\
\mathbf{B} \\
\mathbf{B} \\
\mathbf{E} \\
\mathbf{A}\end{array}$ & $\begin{array}{l}8306.27 \\
8307.41 \\
8311.71 \\
8312.89 \\
8334.42\end{array}$ & $\begin{array}{l}2, \mathrm{~h} \\
\frac{1}{2} \\
2, \mathrm{Co} ? \\
2\end{array}$ & $\begin{array}{l}\mathbf{E} \\
\mathbf{E} \\
\mathbf{E} \\
\mathbf{E} \\
\mathbf{E}\end{array}$ \\
\hline $\begin{array}{l}5752.89 \\
5762.25 \\
5766.33 \\
5774.02 \\
5785.86\end{array}$ & $\begin{array}{l}\text { 1, Co? } \\
2, \mathrm{~h} \\
2, \mathrm{~h} \\
2, \mathrm{~h} \\
2, \mathrm{~h}, \mathrm{Cr} ?\end{array}$ & $\begin{array}{l}\mathbf{B} \\
\mathbf{B} \\
\mathbf{B} \\
\mathbf{B} \\
\mathbf{B}\end{array}$ & $\begin{array}{l}6651.27 \\
6666.60 \\
6681.13 \\
6743.15 \\
6861.47\end{array}$ & $\begin{array}{l}1, n \\
2, n, v \\
1, n \\
2 \\
2\end{array}$ & $\begin{array}{l}\mathbf{E} \\
\mathbf{C} \\
\mathbf{E} \\
\mathbf{A} \\
\mathbf{C}\end{array}$ & $\begin{array}{l}8348.82 \\
8353.12 \\
8364.18 \\
8377.83 \\
8382.61\end{array}$ & $\begin{array}{l}1, h \\
2 \\
2 \\
2 \\
3\end{array}$ & $\begin{array}{l}\mathbf{E} \\
\mathbf{E} \\
\mathbf{A} \\
\mathbf{A} \\
\mathbf{A}\end{array}$ \\
\hline $\begin{array}{l}5804.45 \\
5823.74 \\
5863.10 \\
5866.45 \\
5880.30\end{array}$ & $\begin{array}{l}3, \mathrm{~h} \\
1 \\
1, \mathrm{n} \\
4 \\
1\end{array}$ & $\begin{array}{l}\mathbf{B} \\
\mathbf{B} \\
\mathbf{E} \\
\mathbf{B} \\
\mathbf{B}\end{array}$ & $\begin{array}{l}6996.69 \\
7008.32 \\
7010.94 \\
7035.85 \\
7038.83\end{array}$ & $\begin{array}{l}1 \\
1 \\
1 \\
2 \\
3\end{array}$ & $\begin{array}{l}\mathbf{B} \\
\mathbf{C} \\
\mathbf{B} \\
\mathbf{A} \\
\mathbf{B}\end{array}$ & $\begin{array}{l}8396.85 \\
8412.34 \\
8424.44 \\
8426.46 \\
8434.89\end{array}$ & $\begin{array}{l}2 \\
3 \\
1 \\
4 \\
4\end{array}$ & $\begin{array}{l}\mathbf{A} \\
\mathbf{A} \\
\mathbf{B} \\
\mathbf{A} \\
\mathbf{A}\end{array}$ \\
\hline $\begin{array}{l}5888.70 \\
5899.33 \\
5903.33 \\
5918.54 \\
5922.12\end{array}$ & $\begin{array}{l}1 \\
3 \\
1 \\
2 \\
2\end{array}$ & $\begin{array}{l}\mathbf{E} \\
\mathbf{B} \\
\mathbf{C} \\
\mathbf{A} \\
\mathbf{A}\end{array}$ & $\begin{array}{l}7050.68 \\
7054.38 \\
7069.11 \\
7077.28 \\
7077.88\end{array}$ & $\begin{array}{l}1 \\
4, n \\
2, p ? \\
1 \\
1\end{array}$ & $\begin{array}{l}\mathbf{B} \\
\mathbf{A} \\
\mathbf{A} \\
\mathbf{E} \\
\mathbf{E}\end{array}$ & $\begin{array}{l}8435.64 \\
8438.90 \\
8442.99 \\
8450.89 \\
8457.03\end{array}$ & $\begin{array}{l}5 \\
3 \\
1 \\
3 \\
2, \mathrm{~h}\end{array}$ & $\begin{array}{l}\mathbf{B} \\
\mathbf{B} \\
\mathbf{E} \\
\mathbf{B} \\
\mathbf{E}\end{array}$ \\
\hline $\begin{array}{l}5937.79 \\
5941.73 \\
5953.18 \\
5965.81 \\
5978.56\end{array}$ & $\begin{array}{l}1 \\
2 \\
3 \\
3 \\
3\end{array}$ & $\begin{array}{l}\mathbf{B} \\
\mathbf{A} \\
\mathbf{B} \\
\mathbf{A} \\
\mathbf{A}\end{array}$ & $\begin{array}{l}7087.73 \\
7125.62 \\
7135.54 \\
7138.92 \\
7189.92\end{array}$ & $\begin{array}{l}3, \mathrm{n} \\
3, \mathrm{n} \\
1, \mathrm{Ti} ? \\
1, \mathrm{Ti} \\
2, \mathrm{p} ?\end{array}$ & $\begin{array}{l}\text { A } \\
\text { A } \\
\mathbf{C} \\
\mathbf{B} \\
\mathbf{A}\end{array}$ & $\begin{array}{l}8467.13 \\
8468.45 \\
8495.94 \\
8518.20 \\
8539.30\end{array}$ & $\begin{array}{l}2 \\
3 \\
1 \\
4 \\
1\end{array}$ & $\begin{array}{l}\mathbf{B} \\
\mathbf{B} \\
\mathbf{B} \\
\mathbf{B} \\
\mathbf{B}\end{array}$ \\
\hline $\begin{array}{l}5999.66 \\
6064.64 \\
6070.98 \\
6081.31 \\
6085.24\end{array}$ & $\begin{array}{l}2 \\
2 \\
1 \\
1 \\
3\end{array}$ & $\begin{array}{l}\mathbf{A} \\
\mathbf{B} \\
\mathbf{E} \\
\mathbf{C} \\
\mathbf{B}\end{array}$ & $\begin{array}{l}7209.44 \\
7216.19 \\
7244.82 \\
7251.74 \\
7253.76\end{array}$ & $\begin{array}{l}5 \\
3 \\
4 \\
4 \\
1, p ?\end{array}$ & $\begin{array}{l}\mathbf{A} \\
\mathbf{A} \\
\mathbf{A} \\
\mathbf{A} \\
\mathbf{E}\end{array}$ & $\begin{array}{l}8548.06 \\
8641.55 \\
8675.33 \\
8682.93 \\
8692.29\end{array}$ & $\begin{array}{l}2 \\
1 \\
2 \\
2 \\
1\end{array}$ & $\begin{array}{l}\mathbf{B} \\
\mathbf{E} \\
\mathbf{B} \\
\mathbf{B} \\
\mathbf{B}\end{array}$ \\
\hline $\begin{array}{l}6091.20 \\
6092.79 \\
6098.70 \\
6121.03 \\
6126.22\end{array}$ & $\begin{array}{l}3 \\
1 \\
2 \\
1 \\
3\end{array}$ & $\begin{array}{l}\mathbf{B} \\
\mathbf{B} \\
\mathbf{B} \\
\mathbf{C} \\
\mathbf{B}\end{array}$ & $\begin{array}{l}7299.67 \\
7308.18 \\
7318.43 \\
7330.97 \\
7332.26\end{array}$ & $\begin{array}{l}2 \\
1 \\
2 \\
1, p ? \\
1, p ?\end{array}$ & $\begin{array}{l}\mathbf{E} \\
\mathbf{D} \\
\mathbf{A} \\
\mathbf{E} \\
\mathbf{E}\end{array}$ & $\begin{array}{l}8734.69 \\
8859.70 \\
8989.40 \\
9027.28 \\
9090.68\end{array}$ & $\begin{array}{l}1, \mathbf{h}, \mathrm{Ti} ? \\
1 \\
2 \\
1 \\
1\end{array}$ & $\begin{array}{l}\mathbf{B} \\
\mathbf{E} \\
\mathbf{C} \\
\mathbf{B} \\
\mathbf{B}\end{array}$ \\
\hline $\begin{array}{l}6138.38 \\
6146.22 \\
6148.80 \\
6149.81 \\
6159.02\end{array}$ & $\begin{array}{l}1 \\
2 \\
1, n \\
2, \mathbf{n} \\
2, \mathbf{n}\end{array}$ & $\begin{array}{l}\mathbf{E} \\
\mathbf{B} \\
\mathbf{D} \\
\mathbf{E} \\
\mathbf{B}\end{array}$ & $\begin{array}{l}7344.69 \\
7352.14 \\
7357.73 \\
7364.11 \\
7366.58\end{array}$ & $\begin{array}{l}3 \\
1 \\
3 \\
2 \\
1\end{array}$ & $\begin{array}{l}\mathbf{A} \\
\mathbf{E} \\
\mathbf{A} \\
\mathbf{B} \\
\mathbf{E}\end{array}$ & $\begin{array}{l}9123.09 \\
9167.55 \\
9246.18 \\
9257.62 \\
9285.02\end{array}$ & $\begin{array}{l}1 \\
1 \\
1 \\
1 \\
1\end{array}$ & $\begin{array}{l}\text { B } \\
\text { B } \\
\text { C } \\
\text { C } \\
\text { C }\end{array}$ \\
\hline $\begin{array}{l}6174.47 \\
6186.34 \\
6215.23 \\
6220.45 \\
6221.42\end{array}$ & $\begin{array}{l}2, \mathrm{n} \\
2, \mathrm{n} \\
3, \mathrm{n} \\
2, \mathrm{n} \\
2, \mathrm{p}\end{array}$ & $\begin{array}{l}\mathbf{B} \\
\mathbf{B} \\
\mathbf{B} \\
\mathbf{C} \\
\mathbf{B}\end{array}$ & $\begin{array}{l}7440.58 \\
7474.93 \\
7489.61 \\
7496.12 \\
7614.49\end{array}$ & $\begin{array}{l}3 \\
1, p ? \\
2 \\
2 \\
1, p ?\end{array}$ & $\begin{array}{l}\mathbf{A} \\
\mathbf{E} \\
\mathbf{A} \\
\mathbf{A} \\
\mathbf{E}\end{array}$ & $\begin{array}{l}9546.06 \\
9599.44 \\
9638.26 \\
9647.55\end{array}$ & $\begin{array}{l}1 \\
1 \\
2, \mathrm{~h} \\
1, \mathrm{~h}\end{array}$ & $\begin{array}{l}\mathrm{C} \\
\mathbf{C} \\
\mathrm{B} \\
\mathbf{C}\end{array}$ \\
\hline $\begin{array}{l}6222.56 \\
6258.09 \\
6258.73 \\
6261.08 \\
6275.74\end{array}$ & $\begin{array}{l}2, \mathrm{p} \\
5 \\
5 \\
4 \\
1, \mathrm{~h}\end{array}$ & $\begin{array}{l}\mathbf{E} \\
\mathbf{B} \\
\mathbf{B} \\
\mathbf{B} \\
\mathbf{E}\end{array}$ & $\begin{array}{l}7654.46 \\
7926.34 \\
7943.91 \\
7949.11 \\
7961.59\end{array}$ & $\begin{array}{l}2 \\
1 \\
1, \mathrm{~h}, \mathrm{n} ? \\
3 \\
2\end{array}$ & $\begin{array}{l}\mathbf{B} \\
\mathbf{B} \\
\mathbf{B} \\
\mathbf{A} \\
\mathbf{A}\end{array}$ & $\begin{array}{l}9675.57 \\
975.63 \\
9728.33 \\
9743.58\end{array}$ & $\begin{array}{l}1 \\
1 \\
1 \\
1, \mathrm{~h}\end{array}$ & $\begin{array}{l}\mathbf{B} \\
\mathbf{C} \\
\mathbf{C} \\
\mathbf{C}\end{array}$ \\
\hline
\end{tabular}


Some of Paulson's constant frequency differences have not been confirmed by recent wave-length measurements, ${ }^{6}$ which are more accurate than those of Exner and Haschek, used by Paulson. Similar frequency differences of possibly related lines which are presented here may also be shown to be not strictly constant when more precise measurements are made. The frequencies, or numbers of waves per centimeter, are given for wave lengths as measured in air.

\section{VANADIUM}

Table 2 contains about 250 lines in the arc spectrum of vanadium between the limiting wave lengths $5500 \mathrm{~A}$ and $9522 \mathrm{~A}$. Some of these lines belong to bands, but most of the band structures were not resolved, and therefore not measured. Shaw's ${ }^{7}$ measurements extend from $5800 \mathrm{~A}$ to $7364 \mathrm{~A}$, but only those wave lengths in his list shorter than $6500 \mathrm{~A}$ are given in international units. Vanadium has numerous sharp lines of intensity 2, or greater, in the region from $5500 \mathrm{~A}$ to $6600 \mathrm{~A}$. However, the presence of bands in this region detracts from the desirability of using this portion of the vanadium spectrum as a comparison spectrum for standards.

New pairs of lines with constant frequency differences of the types found by Paulson ${ }^{8}$ are as follows:

\begin{tabular}{|c|r|c|c|}
\hline$\lambda$ & & $\nu$ & $\Delta \nu$ \\
\hline & & & \\
\cline { 1 - 2 } 5507.75 & 3 & $18 ~ 156.23$ & 28.66 \\
5547.06 & 3 & 18027.57 & \\
5707.02 & 10 & 17522.28 & 28.42 \\
5716.29 & 4 & 17493.86 & 28.53 \\
5725.63 & 4 & 17465.33 & \\
5777.68 & 5 & 17459.07 & 28.54 \\
5737.06 & 6 & 17430.53 & \\
5776.65 & 3 & 17311.07 & 28.57 \\
5786.20 & 4 & 17282.50 & \\
6240.20 & 2 & 16025.10 & 29.89 \\
6251.87 & 5 & 15995.21 & \\
6243.06 & 9 & 16017.79 & 29.84 \\
6254.71 & 2 & 15987.95 & \\
6753.03 & 4 & 14808.17 & 29.55 \\
6766.53 & 3 & 14778.62 & \\
6256.91 & 3 & 15982.33 & 30.44 \\
6268.85 & 4 & 15951.89 & \\
6326.88 & 5 & 15805.58 & 30.50 \\
6339.11 & 3 & 15775.09 & \\
\hline
\end{tabular}

${ }^{6}$ Zeit. für Wiss. Phot., 17, pp. 79-142; 1917.

${ }^{8}$ Paulson, Dissertation, L,und; rg14.

7 Astrophysical Journal, 30, p. 127 ; 1909 
TABLE 2.-Arc Spectrum of Vanadium

\begin{tabular}{|c|c|c|c|c|c|c|c|c|}
\hline$\lambda$ I. A. & Notes & $\begin{array}{c}\text { Probable } \\
\text { error }\end{array}$ & $\lambda$ I. A. & Notes & $\begin{array}{c}\text { Probable } \\
\text { error }\end{array}$ & $\lambda$ I. A. & Notes & $\begin{array}{c}\text { Probable } \\
\text { error }\end{array}$ \\
\hline $\begin{array}{l}\mathbf{5 5 0 4 . 8 4} \\
5507.75 \\
5511.11 \\
5547.06 \\
5561.75\end{array}$ & $\begin{array}{l}1 \\
3 \\
1 \\
3 \\
2\end{array}$ & $\begin{array}{l}\mathbf{E} \\
\mathbf{B} \\
\mathbf{E} \\
\mathbf{B} \\
\mathbf{B}\end{array}$ & $\begin{array}{l}6039.76 \\
6048.69 \\
6054.47 \\
6058.15 \\
6063.45\end{array}$ & $\begin{array}{l}6 \\
1 \\
1 \\
4 \\
1\end{array}$ & $\begin{array}{l}\mathbf{A} \\
\mathbf{A} \\
\mathbf{A} \\
\mathbf{A} \\
\mathbf{A}\end{array}$ & $\begin{array}{l}6543.53 \\
6550.13 \\
6558.05 \\
6565.88 \\
6568.81\end{array}$ & $\begin{array}{l}2 \\
\frac{1}{2}, \mathrm{v} ? \\
2 \\
1\end{array}$ & $\begin{array}{l}\mathbf{A} \\
\mathbf{A} \\
\mathbf{A} \\
\mathbf{A} \\
\mathbf{A}\end{array}$ \\
\hline $\begin{array}{l}\mathbf{5 5 8 4 . 5 5} \\
5592.48 \\
5601.48 \\
5604.94 \\
\mathbf{5 6 2 2 . 1 1}\end{array}$ & $\begin{array}{l}5 \\
5 \\
1 \\
6 \\
1\end{array}$ & $\begin{array}{l}\mathbf{B} \\
\mathbf{B} \\
\mathbf{B} \\
\mathbf{B} \\
\mathbf{E}\end{array}$ & $\begin{array}{l}6067.25 \\
6081.46 \\
6086.67 \\
6087.67 \\
6090.25\end{array}$ & $\begin{array}{l}2 \\
6 \\
1, n \\
\frac{1}{7}\end{array}$ & $\begin{array}{l}\mathbf{B} \\
\mathbf{A} \\
\mathbf{C} \\
\mathbf{E} \\
\mathbf{B}\end{array}$ & $\begin{array}{l}6579.00 \\
6605.98 \\
6607.83 \\
6623.59 \\
6624.87\end{array}$ & $\begin{array}{l}1 \\
3 \\
2 \\
1 \\
3\end{array}$ & $\begin{array}{l}\mathbf{B} \\
\mathbf{A} \\
\mathbf{A} \\
\mathbf{B} \\
\mathbf{A}\end{array}$ \\
\hline $\begin{array}{l}5624.69 \\
5626.06 \\
5627.67 \\
5632.43 \\
5635.56\end{array}$ & $\begin{array}{l}8 \\
3 \\
8 \\
1 \\
1\end{array}$ & $\begin{array}{l}\mathbf{B} \\
\mathbf{B} \\
\mathbf{A} \\
\mathbf{E} \\
\mathbf{E}\end{array}$ & $\begin{array}{l}6104.65 \\
6106.97 \\
6111.68 \\
6119.56 \\
6135.37\end{array}$ & $\begin{array}{l}1 \\
1 \\
6 \\
6 \\
5\end{array}$ & $\begin{array}{l}\mathbf{E} \\
\mathbf{A} \\
\mathbf{A} \\
\mathbf{A} \\
\mathbf{A}\end{array}$ & $\begin{array}{l}6633.35 \\
6693.65 \\
6708.03 \\
6753.03 \\
6760.12\end{array}$ & $\begin{array}{l}1 \\
1 \\
1 \\
4 \\
2\end{array}$ & $\begin{array}{l}\mathbf{B} \\
\mathbf{B} \\
\mathbf{B} \\
\mathbf{A} \\
\mathbf{A}\end{array}$ \\
\hline $\begin{array}{l}5646.13 \\
5657.45 \\
5668.38 \\
5670.88 \\
5683.32\end{array}$ & $\begin{array}{l}5 \\
7 \\
5 \\
7 \\
3\end{array}$ & $\begin{array}{l}\mathbf{A} \\
\mathbf{B} \\
\mathbf{B} \\
\mathbf{A} \\
\mathbf{B}\end{array}$ & $\begin{array}{l}6150.18 \\
6170.39 \\
6189.37 \\
6199.21 \\
6213.90\end{array}$ & $\begin{array}{l}5 \\
3 \\
2 \\
4 \\
2\end{array}$ & $\begin{array}{l}\mathbf{B} \\
\mathbf{B} \\
\mathbf{B} \\
\mathbf{B} \\
\mathbf{B}\end{array}$ & $\begin{array}{l}6766.53 \\
6785.02 \\
6786.36 \\
6812.42 \\
6832.47\end{array}$ & $\begin{array}{l}3 \\
2 \\
1 \\
2 \\
2\end{array}$ & $\begin{array}{l}\mathbf{A} \\
\mathbf{A} \\
\mathbf{B} \\
\mathbf{A} \\
\mathbf{A}\end{array}$ \\
\hline $\begin{array}{l}5687.83 \\
5691.29 \\
5698.52 \\
5703.60 \\
5707.02\end{array}$ & $\begin{array}{r}2 \\
2 \\
9 \\
10 \\
10\end{array}$ & $\begin{array}{l}\text { B } \\
\mathbf{C} \\
\text { A } \\
\text { B } \\
\text { B }\end{array}$ & $\begin{array}{l}6216.38 \\
6218.36 \\
6224.53 \\
6230.80 \\
6233.20\end{array}$ & $\begin{array}{l}5 \\
2 \\
3 \\
7 \\
4\end{array}$ & $\begin{array}{l}\mathbf{A} \\
\mathbf{A} \\
\mathbf{A} \\
\mathbf{A} \\
\mathbf{A}\end{array}$ & $\begin{array}{l}6839.59 \\
6841.90 \\
6870.86 \\
6871.55 \\
6894.00\end{array}$ & $\begin{array}{l}2 \\
2 \\
2 \\
2 \\
1, \mathrm{n}\end{array}$ & $\begin{array}{l}\mathbf{A} \\
\mathbf{A} \\
\mathbf{A} \\
\mathbf{B} \\
\mathbf{B}\end{array}$ \\
\hline $\begin{array}{l}5708.94 \\
5716.29 \\
5725.63 \\
5727.02 \\
5727.68\end{array}$ & $\begin{array}{l}1 \\
4 \\
4 \\
7 \\
5\end{array}$ & $\begin{array}{l}\mathbf{A} \\
\mathrm{B} \\
\mathrm{B} \\
\mathrm{B} \\
\mathbf{B}\end{array}$ & $\begin{array}{l}6240.20 \\
6243.06 \\
6245.26 \\
6251.87 \\
6254.71\end{array}$ & $\begin{array}{l}2 \\
9 \\
2 \\
5 \\
2\end{array}$ & $\begin{array}{l}\text { A } \\
\text { A } \\
\text { B } \\
\text { A } \\
\text { E }\end{array}$ & $\begin{array}{l}6974.56 \\
7026.06 \\
7264.24 \\
7321.47 \\
7338.93\end{array}$ & $\begin{array}{l}1 \\
1 \\
2 \\
1 \\
3\end{array}$ & $\begin{array}{l}\mathbf{A} \\
\mathbf{B} \\
\mathbf{B} \\
\mathbf{B} \\
\mathbf{A}\end{array}$ \\
\hline $\begin{array}{l}5731.28 \\
5733.24 \\
5734.01 \\
5737.06 \\
5743.45\end{array}$ & $\begin{array}{l}6 \\
1 \\
3 \\
6 \\
5\end{array}$ & $\begin{array}{l}\mathbf{A} \\
\mathbf{B} \\
\mathbf{A} \\
\mathbf{A} \\
\mathbf{B}\end{array}$ & $\begin{array}{l}6256.91 \\
6258.58 \\
6261.25 \\
6266.32 \\
6268.85\end{array}$ & $\begin{array}{l}3 \\
3 \\
3 \\
3 \\
4\end{array}$ & $\begin{array}{l}\mathbf{A} \\
\mathbf{A} \\
\mathbf{A} \\
\mathbf{A} \\
\mathbf{A}\end{array}$ & $\begin{array}{l}7345.11 \\
7356.50 \\
7358.62 \\
7361.39 \\
7362.55\end{array}$ & $\begin{array}{l}2, \mathrm{n} \\
2 \\
1 \\
2 \\
1\end{array}$ & $\begin{array}{l}\text { B } \\
\text { A } \\
\text { A } \\
\text { A } \\
\text { B }\end{array}$ \\
\hline $\begin{array}{l}5747.73 \\
5748.84 \\
5750.67 \\
5755.15 \\
5761.40\end{array}$ & $\begin{array}{l}1 \\
2 \\
1 \\
1 \\
1\end{array}$ & $\begin{array}{l}\text { A } \\
\text { B } \\
\text { B } \\
\text { C } \\
\text { B }\end{array}$ & $\begin{array}{l}6274.68 \\
6282.34 \\
6285.18 \\
6286.91 \\
6292.85\end{array}$ & $\begin{array}{l}5 \\
1 \\
4 \\
1 \\
5\end{array}$ & $\begin{array}{l}\mathrm{A} \\
\mathrm{A} \\
\mathrm{B} \\
\mathrm{E} \\
\mathrm{A}\end{array}$ & $\begin{array}{l}7363.17 \\
7393.39 \\
7431.50 \\
7434.00 \\
7435.74\end{array}$ & $\begin{array}{l}2 \\
2, \mathrm{n} \\
2, \mathrm{p} \\
2, \mathrm{p} \\
2, \mathrm{n}\end{array}$ & $\begin{array}{l}\mathbf{B} \\
\mathbf{E} \\
\mathbf{E} \\
\mathbf{E} \\
\mathbf{E}\end{array}$ \\
\hline $\begin{array}{l}5772.44 \\
5776.65 \\
5782.71 \\
5783.52 \\
5784.45\end{array}$ & $\begin{array}{l}3 \\
3 \\
1 \\
1 \\
3\end{array}$ & $\begin{array}{l}\text { A } \\
\text { B } \\
\text { B } \\
\text { B } \\
\text { B }\end{array}$ & $\begin{array}{l}6295.20 \\
6296.53 \\
6304.38 \\
6311.53 \\
6321.26\end{array}$ & $\begin{array}{l}1 \\
5 \\
1 \\
2, b \\
1\end{array}$ & $\begin{array}{l}\mathbf{E} \\
\mathbf{A} \\
\mathbf{C} \\
\mathbf{A} \\
\mathbf{B}\end{array}$ & $\begin{array}{l}7578.78 \\
7790.57 \\
7801.78 \\
7851.16 \\
7865.51\end{array}$ & $\begin{array}{l}1 \\
2, \mathrm{~h} \\
1 \\
2 \\
2, \mathrm{n}\end{array}$ & $\begin{array}{l}B \\
C \\
C \\
C \\
C\end{array}$ \\
\hline $\begin{array}{l}5786.20 \\
5788.52 \\
5799.88 \\
5807.16 \\
5817.14\end{array}$ & $\begin{array}{l}4 \\
3 \\
1 \\
1 \\
1\end{array}$ & $\begin{array}{l}\text { B } \\
\text { A } \\
\text { A } \\
\text { B } \\
\text { B }\end{array}$ & $\begin{array}{l}6324.69 \\
6326.88 \\
6339.11 \\
6349.50 \\
6355.66\end{array}$ & $\begin{array}{l}1 \\
5 \\
3 \\
3 \\
2\end{array}$ & $\begin{array}{l}\text { B } \\
\mathbf{B} \\
\mathbf{B} \\
\mathbf{A} \\
\mathbf{A}\end{array}$ & $\begin{array}{l}7898.81 \\
8027.34 \\
8093.47 \\
8102.37 \\
8108.72\end{array}$ & $\begin{array}{l}1 \\
2 \\
2 \\
2 \\
2\end{array}$ & $\begin{array}{l}\mathrm{E} \\
\mathrm{B} \\
\mathrm{B} \\
\mathrm{E} \\
\mathbf{C}\end{array}$ \\
\hline $\begin{array}{l}5817.61 \\
5830.75 \\
5846.35 \\
5850.35 \\
5924.59\end{array}$ & $\begin{array}{l}2 \\
4 \\
6 \\
1 \\
3\end{array}$ & $\begin{array}{l}\text { B } \\
\text { B } \\
\text { B } \\
\text { B } \\
\text { B }\end{array}$ & $\begin{array}{l}6357.31 \\
6358.80 \\
6361.26 \\
6379.35 \\
6393.30\end{array}$ & $\begin{array}{l}3 \\
3 \\
2 \\
2 \\
2\end{array}$ & $\begin{array}{l}\mathbf{A} \\
\mathbf{A} \\
\mathbf{B} \\
\mathbf{A} \\
\mathbf{A}\end{array}$ & $\begin{array}{l}8109.01 \\
8109.84 \\
8116.78 \\
8144.52 \\
8154.52\end{array}$ & $\begin{array}{l}2 \\
1 \\
5 \\
3 \\
2\end{array}$ & $\begin{array}{l}\text { C } \\
\text { E } \\
\text { A } \\
\text { B } \\
\text { A }\end{array}$ \\
\hline $\begin{array}{l}5975.42 \\
5978.94 \\
5980.81 \\
5984.74 \\
6002.51\end{array}$ & $\begin{array}{l}1 \\
2 \\
1 \\
1 \\
3, \mathrm{C} 0 ? \mathrm{~d} ?\end{array}$ & $\begin{array}{l}\text { C } \\
\text { B } \\
\text { B } \\
\text { C } \\
\text { B }\end{array}$ & $\begin{array}{l}6430.49 \\
6431.68 \\
6433.15 \\
6435.19 \\
6445.21\end{array}$ & $\begin{array}{l}1 \\
1 \\
2 \\
2 \\
1\end{array}$ & $\begin{array}{l}\text { A } \\
\text { A } \\
\text { B } \\
\text { B } \\
\text { C }\end{array}$ & $\begin{array}{l}8161.06 \\
8171.30 \\
8186.71 \\
8187.38 \\
8198.85\end{array}$ & $\begin{array}{l}4 \\
3 \\
3 \\
3 \\
3 \\
3\end{array}$ & $\begin{array}{l}\mathbf{A} \\
\mathbf{A} \\
\mathbf{B} \\
\mathbf{B} \\
\mathbf{B}\end{array}$ \\
\hline $\begin{array}{l}6008.69 \\
6016.13 \\
6017.95 \\
6024.22 \\
6025.43\end{array}$ & $\begin{array}{l}1 \\
2 \\
2 \\
1 \\
2\end{array}$ & $\begin{array}{l}\mathbf{E} \\
\mathbf{B} \\
\mathbf{A} \\
\mathbf{E} \\
\mathbf{A}\end{array}$ & $\begin{array}{l}6452.38 \\
6477.55 \\
6488.12 \\
6504.18 \\
6531.43\end{array}$ & $\begin{array}{l}4 \\
1, n \\
2 \\
4 \\
5\end{array}$ & $\begin{array}{l}\mathbf{B} \\
\mathbf{E} \\
\mathbf{E} \\
\mathbf{A} \\
\mathbf{A}\end{array}$ & $\begin{array}{l}8203.11 \\
8241.60 \\
8253.48 \\
8255.90 \\
8280.37\end{array}$ & $\begin{array}{l}4 \\
4 \\
4 \\
4 \\
1\end{array}$ & $\begin{array}{l}\mathbf{B} \\
\mathbf{A} \\
\mathbf{A} \\
\mathbf{A} \\
\mathbf{A}\end{array}$ \\
\hline
\end{tabular}


TABLE 2-Continued

\begin{tabular}{|c|c|c|c|c|c|c|c|c|}
\hline$\lambda$ I. A. & Notes & $\begin{array}{c}\text { Probable } \\
\text { error }\end{array}$ & $\lambda$ I. A. & Notes & $\begin{array}{c}\text { Probable } \\
\text { error }\end{array}$ & $\lambda$ I. A. & Notes & $\begin{array}{c}\text { Probable } \\
\text { error }\end{array}$ \\
\hline $\begin{array}{l}8282.36 \\
8324.31 \\
8331.23 \\
8342.06 \\
8402.78\end{array}$ & $\begin{array}{l}3 \\
2 \\
2 \\
2 \\
2\end{array}$ & $\begin{array}{l}\text { A } \\
\text { B } \\
\text { B } \\
\text { B } \\
\text { B }\end{array}$ & $\begin{array}{l}8624.90 \\
8657.83 \\
8762.37 \\
8919.78 \\
8932.93\end{array}$ & $\begin{array}{l}2,1 \\
1 \\
1, \mathbf{n} \\
3 \\
2\end{array}$ & $\begin{array}{l}\text { C } \\
\text { E } \\
\text { E } \\
\text { B } \\
\text { A }\end{array}$ & $\begin{array}{l}9226.10 \\
9242.96 \\
9255.91 \\
9264.47 \\
9265.69\end{array}$ & $\begin{array}{l}1, \mathbf{h} \\
1 \\
1 \\
1 \\
1\end{array}$ & $\begin{array}{l}\mathbf{E} \\
\mathbf{E} \\
\mathbf{E} \\
\mathbf{E} \\
\mathbf{E}\end{array}$ \\
\hline $\begin{array}{l}8408.24 \\
8414.42 \\
8416.50 \\
8421.37 \\
8431.62\end{array}$ & $\begin{array}{l}\mathbf{1}, \mathbf{h} \\
1 \\
1 \\
1 \\
1\end{array}$ & $\begin{array}{l}\text { C } \\
\text { B } \\
\text { B } \\
\text { B } \\
\text { B }\end{array}$ & $\begin{array}{l}8971.65 \\
9003.02 \\
9021.10 \\
9022.72 \\
9037.55\end{array}$ & $\begin{array}{l}1 \\
1 \\
2 \\
2 \\
2\end{array}$ & $\begin{array}{l}\text { A } \\
\text { E } \\
\text { B } \\
\text { B } \\
\text { B }\end{array}$ & $\begin{array}{l}9273.37 \\
9308.80 \\
9328.27 \\
9341.06\end{array}$ & $\begin{array}{l}1 \\
1 \\
1 \\
1\end{array}$ & $\begin{array}{l}\mathbf{E} \\
\mathbf{E} \\
\mathbf{E} \\
\mathbf{C}\end{array}$ \\
\hline $\begin{array}{l}8468.17 \\
8493.62 \\
849.51 \\
8502.94 \\
8505.55\end{array}$ & $\begin{array}{l}1, p \\
1 \\
2 \\
1 \\
2, v\end{array}$ & $\begin{array}{l}\text { E } \\
\text { B } \\
\text { B } \\
\text { C } \\
\text { B }\end{array}$ & $\begin{array}{l}9046.69 \\
9073.34 \\
9085.23 \\
9100.74 \\
9105.68\end{array}$ & $\begin{array}{l}2 \\
1 \\
.2, \mathbf{n} \\
1, \mathbf{b} \\
1\end{array}$ & $\begin{array}{l}\mathrm{B} \\
\mathrm{E} \\
\mathrm{B} \\
\mathrm{B} \\
\mathrm{B} \\
\mathrm{D}\end{array}$ & $\begin{array}{l}9366.92 \\
9385.15 \\
9435.58 \\
9521.87\end{array}$ & & $\begin{array}{l}\mathbf{E} \\
\mathbf{E} \\
\mathbf{E} \\
\mathbf{E}\end{array}$ \\
\hline $\begin{array}{l}8534.52 \\
8538.19 \\
8580.53 \\
8590.97 \\
8604.98\end{array}$ & $\begin{array}{l}2 \\
1, \mathrm{n} \\
1, \mathrm{p} ? \\
1, \mathrm{~b} \\
1, \mathrm{~b}\end{array}$ & $\begin{array}{l}\mathrm{B} \\
\mathrm{E} \\
\mathrm{E} \\
\mathrm{E} \\
\mathrm{E}\end{array}$ & $\begin{array}{l}9113.76 \\
9141.25 \\
915.48 \\
9164.80 \\
9168.83\end{array}$ & $\begin{array}{l}1 \\
1 \\
1 \\
1 \\
1\end{array}$ & $\begin{array}{l}\text { E } \\
\text { E } \\
\text { D } \\
\text { B } \\
\text { C }\end{array}$ & & & \\
\hline
\end{tabular}

\section{CHROMIUM}

The red and infra-red spectrum of chromium is characterized by the presence of several complex triplets of strong lines and a number of bands, presumably due to the oxide. In Table 3 are given I 30 wave lengths between the limits $55^{64} \mathrm{~A}$ and $9735 \mathrm{~A}$. Stüting's ${ }^{9}$ work, based on the standard iron lines of Fabry and Buisson, extends from 5902 A to 7463 A. Meissner's ${ }^{10}$ measurements refer orrly to the triplets in the red and infra-red regions, and the values given for them in the following table are in close agreement with his. Observing with a bolometer, Randall and Barker ${ }^{11}$ have found many infra-red lines in the spectrum of chromium, beginning at wave length $8947 \mathrm{~A}$. All of their lines up to $9732 \mathrm{~A}$ are to be found in Table 3 .

The frequency differences of two sets of triplets in the red region of the spectrum have been calculated, and it is found that they are enantiomorphs of two other sets of shorter wave lengths. The data are as follows:

\begin{tabular}{|c|c|c|c|c|c|c|c|}
\hline$\lambda$ & & $\nu$ & $\Delta \nu$ & $\lambda$ & & $\nu$ & $\Delta \nu$ \\
\hline $\begin{array}{l}4254.34 \\
4274.80 \\
4289.72\end{array}$ & $\begin{array}{l}12 \\
12 \\
10\end{array}$ & $\begin{array}{ll}23 & 505.41 \\
23 & 392.91 \\
23 & 311.54\end{array}$ & $\begin{array}{r}112.50 \\
81.37\end{array}$ & $\begin{array}{l}7355.93 \\
7400.22 \\
7462.34\end{array}$ & $\begin{array}{l}10 \\
10 \\
10\end{array}$ & $\begin{array}{ll}13 & 594.47 \\
13 & 513.11 \\
13 & 400.62\end{array}$ & $\begin{array}{r}81.36 \\
112.49\end{array}$ \\
\hline $\begin{array}{l}5204.51 \\
5206.05 \\
5208.42\end{array}$ & $\begin{array}{l}12, \mathbf{R} \\
12, \mathbf{R} \\
12, \mathbf{R}\end{array}$ & $\begin{array}{ll}19 & 214.10 \\
19 & 208.42 \\
19 & 199.68\end{array}$ & $\begin{array}{l}5.68 \\
8.74\end{array}$ & $\begin{array}{l}9009.95 \\
9017.10 \\
9021.69\end{array}$ & $\begin{array}{l}6 \\
5 \\
4\end{array}$ & $\begin{array}{ll}11 & 098.84 \\
11 & 090.04 \\
11 & 084.40\end{array}$ & $\begin{array}{l}8.80 \\
5.64\end{array}$ \\
\hline
\end{tabular}


The wave lengths of the two triplets in the left-hand column are taken from Kayser's Handbuch.

TABLE 3.-Arc Spectrum of Chromium

\begin{tabular}{|c|c|c|c|c|c|c|c|c|}
\hline$\lambda$ I. A. & Notes & $\begin{array}{c}\text { Probable } \\
\text { error }\end{array}$ & $\lambda$ I. A. & Notes & $\begin{array}{c}\text { Probable } \\
\text { error }\end{array}$ & $\lambda$ I. A. & Notes & $\begin{array}{c}\text { Probable } \\
\text { error }\end{array}$ \\
\hline $\begin{array}{l}5564.58 \\
5628.64 \\
5642.34 \\
5648.12 \\
5649.41\end{array}$ & $\begin{array}{l}3, \mathrm{n} \\
3 \\
2, \mathrm{p} ? \\
2, \mathrm{p} ? \\
2, \mathrm{p} ?\end{array}$ & $\begin{array}{l}\text { D } \\
\text { A } \\
D \\
\text { D } \\
\text { B }\end{array}$ & $\begin{array}{l}6630.01 \\
6642.99 \\
6661.09 \\
6663.93 \\
6669.25\end{array}$ & $\begin{array}{l}3 \\
1 \\
5 \\
1, p ? \\
4\end{array}$ & $\begin{array}{l}\mathbf{A} \\
\mathbf{E} \\
\mathbf{A} \\
\mathbf{E} \\
\mathbf{A}\end{array}$ & $\begin{array}{l}7990.51 \\
8007.97 \\
8061.29 \\
8084.98 \\
8163.21\end{array}$ & $\begin{array}{l}1, \mathrm{~h} \\
1, \mathrm{~h} \\
1 \\
3\end{array}$ & $\begin{array}{l}\text { A } \\
\mathbf{B} \\
\mathbf{B} \\
\text { A } \\
\text { B }\end{array}$ \\
\hline $\begin{array}{l}5664.10 \\
5681.21 \\
5682.50 \\
5683.60 \\
5694.81\end{array}$ & $\begin{array}{l}4 \\
2 \\
3 \\
1 \\
5\end{array}$ & $\begin{array}{l}\mathbf{A} \\
\mathbf{C} \\
\mathbf{C} \\
\mathbf{B} \\
\mathbf{B}\end{array}$ & $\begin{array}{l}6677.20 \\
6680.18 \\
6715.36 \\
6729.74 \\
6734.16\end{array}$ & $\begin{array}{l}2, \mathrm{p} ? \\
2, \mathrm{p} ? \\
3, \mathrm{~h} \\
3, \mathrm{~h} \\
2\end{array}$ & $\begin{array}{l}\mathbf{C} \\
\mathbf{C} \\
\mathbf{A} \\
\mathbf{A} \\
\mathbf{A}\end{array}$ & $\begin{array}{l}8166.64 \\
8235.90 \\
8238.26 \\
8287.38 \\
8290.59\end{array}$ & $\begin{array}{l}1 \\
2 \\
1 \\
1 \\
1, \mathrm{~h}\end{array}$ & $\begin{array}{l}\mathbf{D} \\
\mathbf{B} \\
\mathbf{B} \\
\mathbf{B} \\
\mathbf{D}\end{array}$ \\
\hline $\begin{array}{l}5698.33 \\
5702.31 \\
5712.75 \\
5719.87 \\
5729.23\end{array}$ & $\begin{array}{l}5 \\
4 \\
4 \\
2 \\
1, p ?\end{array}$ & $\begin{array}{l}\mathbf{B} \\
\mathbf{A} \\
\mathbf{A} \\
\mathbf{A} \\
\mathbf{D}\end{array}$ & $\begin{array}{l}6744.65 \\
6746.65 \\
675.28 \\
6757.77 \\
6762.42\end{array}$ & $\begin{array}{l}1 \\
1 \\
2 \\
2 \\
2\end{array}$ & $\begin{array}{l}\mathbf{B} \\
\mathbf{C} \\
\mathbf{A} \\
\mathbf{A} \\
\mathbf{A}\end{array}$ & $\begin{array}{l}8348.27 \\
8450.25 \\
8455.22 \\
8483.45 \\
8511.08\end{array}$ & $\begin{array}{l}2 \\
2 \\
2 \\
1, \mathrm{~h} \\
1\end{array}$ & $\begin{array}{l}\mathbf{B} \\
\mathbf{B} \\
\mathbf{C} \\
\mathrm{E} \\
\mathrm{E}\end{array}$ \\
\hline $\begin{array}{l}5738.54 \\
5746.44 \\
5753.68 \\
5771.62 \\
5781.09\end{array}$ & $\begin{array}{l}1 \mathrm{p} ? \\
1, \mathrm{p} ? \\
2, \mathrm{~h} \\
\mathbf{2}, \mathbf{h}\end{array}$ & $\begin{array}{l}\mathbf{B} \\
\mathbf{A} \\
\mathbf{A} \\
\mathbf{C} \\
\mathbf{B}\end{array}$ & $\begin{array}{l}6772.37 \\
6828.81 \\
6830.42 \\
6881.62 \\
6882.38\end{array}$ & $\begin{array}{l}2, n \\
2, n \\
2, n \\
9 \\
9\end{array}$ & $\begin{array}{l}\mathbf{A} \\
\mathbf{E} \\
\mathbf{A} \\
\mathbf{A} \\
\mathbf{A}\end{array}$ & $\begin{array}{l}8537.89 \\
8548.82 \\
8555.55 \\
8562.63 \\
8583.07\end{array}$ & $\begin{array}{l}1 \\
2 \\
1 \\
1, \mathrm{~h} \\
1\end{array}$ & $\begin{array}{l}\mathbf{E} \\
\mathbf{A} \\
\mathbf{D} \\
\mathbf{E} \\
\mathbf{E}\end{array}$ \\
\hline $\begin{array}{l}\mathbf{5 7 8 1 . 8 1} \\
5783.12 \\
5783.95 \\
5785.01 \\
\mathbf{5 7 8 5 . 8 2}\end{array}$ & $\begin{array}{l}9 \\
9 \\
9 \\
8 \\
8\end{array}$ & $\begin{array}{l}\text { B } \\
\text { A } \\
\text { A } \\
\text { A }\end{array}$ & $\begin{array}{l}6883.03 \\
6924.14 \\
6925.20 \\
6925.99 \\
6978.51\end{array}$ & $\begin{array}{c}9 \\
10 \\
9 \\
1 \\
10\end{array}$ & $\begin{array}{l}\mathbf{A} \\
\mathbf{A} \\
\mathbf{A} \\
\mathbf{A} \\
\mathbf{A}\end{array}$ & $\begin{array}{l}8636.32 \\
8643.02 \\
8687.55 \\
8707.48 \\
8707.98\end{array}$ & $\begin{array}{l}1 \\
1 \\
1 \\
1 \\
1\end{array}$ & $\begin{array}{l}\mathbf{E} \\
\mathbf{D} \\
\mathbf{E} \\
\mathbf{E} \\
\mathbf{E}\end{array}$ \\
\hline $\begin{array}{l}5787.06 \\
5788.01 \\
5791.00 \\
5794.71 \\
5796.76\end{array}$ & $\begin{array}{c}2 \\
9 \\
10 \\
3, n \\
1, \mathrm{p} ?\end{array}$ & $\begin{array}{l}\text { B } \\
\text { A } \\
\text { A } \\
\text { C } \\
\text { E }\end{array}$ & $\begin{array}{l}6979.79 \\
6980.81 \\
6981.04 \\
7170.063 \\
7185.51\end{array}$ & $\begin{array}{l}7 \\
2 \\
3 \\
2, p ? \\
2\end{array}$ & $\begin{array}{l}\mathbf{A} \\
\mathbf{A} \\
\mathbf{B} \\
\mathbf{B} \\
\mathbf{A}\end{array}$ & $\begin{array}{l}8718.78 \\
8947.21 \\
8976.88 \\
9009.95 \\
9017.10\end{array}$ & $\begin{array}{l}1 \\
2 \\
3 \\
6 \\
5\end{array}$ & $\begin{array}{l}\mathbf{E} \\
\mathbf{B} \\
\mathbf{A} \\
\mathbf{B} \\
\mathbf{B}\end{array}$ \\
\hline $\begin{array}{l}5844.69 \\
5854.21 \\
5866.94 \\
5902.13 \\
6051.91\end{array}$ & $\begin{array}{l}2, \mathrm{p} ? \\
2, \mathrm{p} ? \\
2, \mathrm{p} ? \\
1, \mathrm{p} ? \\
3, \mathrm{n}\end{array}$ & $\begin{array}{l}\mathrm{E} \\
\mathrm{E} \\
\mathrm{E} \\
\mathrm{E} \\
\mathrm{B}\end{array}$ & $\begin{array}{l}7188.07 \\
7355.93 \\
7400.22 \\
7462.34 \\
7722.88\end{array}$ & $\begin{array}{l}1 \\
10 \\
10 \\
10 \\
2\end{array}$ & $\begin{array}{l}\mathbf{B} \\
\mathbf{A} \\
\mathbf{A} \\
\mathbf{A} \\
\mathbf{C}\end{array}$ & $\begin{array}{l}9021.69 \\
9035.88 \\
9140.37 \\
9141.13 \\
9142.62\end{array}$ & $\begin{array}{l}4 \\
3 \\
1, \mathrm{~h} \\
1, \mathrm{~h} \\
1, \mathrm{~h}\end{array}$ & $\begin{array}{l}\mathrm{B} \\
\mathrm{B} \\
\mathbf{C} \\
\mathrm{B} \\
\mathrm{C}\end{array}$ \\
\hline $\begin{array}{l}6135.75 \\
6190.64 \\
6330.10 \\
6362.83 \\
6394.64\end{array}$ & $\begin{array}{l}2, \mathrm{p} ? \\
2, \mathrm{p} ? \\
4 \\
4 \\
3, \mathrm{n}\end{array}$ & $\begin{array}{l}\mathbf{E} \\
\mathbf{E} \\
\mathbf{A} \\
\mathbf{A} \\
\mathbf{A}\end{array}$ & $\begin{array}{l}7724.63 \\
7726.00 \\
7762.99 \\
7861.30 \\
7885.01\end{array}$ & $\begin{array}{l}1 \\
1 \\
1 \\
1 \\
1\end{array}$ & $\begin{array}{l}\mathrm{E} \\
\mathrm{B} \\
\mathrm{B} \\
\mathrm{E} \\
\mathrm{E}\end{array}$ & $\begin{array}{l}9208.33 \\
9263.92 \\
9290442 \\
9294.11 \\
9447.00\end{array}$ & $\begin{array}{l}2 \\
2,0 ? \\
4 \\
2 \\
3\end{array}$ & $\begin{array}{l}\text { C } \\
\text { C } \\
\text { B } \\
\text { C }\end{array}$ \\
\hline $\begin{array}{l}6537.95 \\
6572.90 \\
6594.71 \\
6597.55 \\
6612.15\end{array}$ & $\begin{array}{l}2 \\
2, p ? \\
2 \\
2 \\
2\end{array}$ & $\begin{array}{l}\mathbf{B} \\
\mathbf{A} \\
\mathbf{A} \\
\mathbf{A} \\
\mathbf{A}\end{array}$ & $\begin{array}{l}7908.30 \\
7910.48 \\
7917.84 \\
7942.00 \\
7989.37\end{array}$ & $\begin{array}{l}2 \\
1 \\
1 \\
2 \\
1\end{array}$ & $\begin{array}{l}\mathbf{A} \\
\mathbf{A} \\
\mathbf{A} \\
\mathbf{A} \\
\mathbf{A}\end{array}$ & $\begin{array}{l}9571.70 \\
9574.22 \\
9670.46 \\
9734.51\end{array}$ & $\begin{array}{l}0 \\
2 \\
1 \\
1\end{array}$ & $\begin{array}{l}\mathrm{C} \\
\mathrm{C} \\
\mathrm{D} \\
\mathrm{C}\end{array}$ \\
\hline
\end{tabular}

\section{MANGANESE}

In the arc spectrum of manganese I93 lines ('Table 4 ) have been measured between the limits $5500 \mathrm{~A}$ and $9600 \mathrm{~A}$. Wave lengths in the international code in the red region of the spectrum have been published by Kilby, ${ }^{12}$ whose measurements extend to $6022 \mathrm{~A}$, and by Fuchs, ${ }^{13}$ whose measurements extend to $7070 \mathrm{~A}$. In the infra-red region Randall and Barker ${ }^{14}$ give three lines which are included in our list. 
Among the lines of Table 4, two new pairs have been found with constant frequency differences of the type found by Paulson..$^{15}$ They are as follows:

\begin{tabular}{|c|c|c|c|}
\hline$\lambda$ & & $\nu$ & $\Delta \nu$ \\
\hline 5505.88 & 3 & 18162.40 & 35.85 \\
5516.77 & 7 & 18126.55 & \\
7283.80 & 6 & 13729.10 & 35.95 \\
7302.92 & 6 & 13693.15 & \\
\hline
\end{tabular}

TABLE 4.-Arc Spectrum of Manganese

\begin{tabular}{|c|c|c|c|c|c|c|c|c|}
\hline$\lambda$ I. A. & Notes & $\begin{array}{c}\text { Probable } \\
\text { error }\end{array}$ & $\lambda$ I. A. & Notes & $\begin{array}{c}\text { Probable } \\
\text { error }\end{array}$ & $\lambda$ I. A. & Notes & $\begin{array}{c}\text { Probable } \\
\text { error }\end{array}$ \\
\hline $\begin{array}{l}5505.88 \\
5516.77 \\
5520.49 \\
5537.70 \\
5543.44\end{array}$ & $\begin{array}{l}3 \\
7 \\
1 \\
7 \\
1\end{array}$ & $\begin{array}{l}\text { B } \\
\text { B } \\
\text { B } \\
\text { C } \\
\text { E }\end{array}$ & $\begin{array}{l}6349.75 \\
6356.08 \\
6370.81 \\
6378.94 \\
6382.18\end{array}$ & $\begin{array}{l}3 \\
2 \\
2, \mathrm{~h} \\
3 \\
3\end{array}$ & $\begin{array}{l}\mathrm{B} \\
\mathrm{B} \\
\mathrm{B} \\
\mathrm{B} \\
\mathrm{B}\end{array}$ & $\begin{array}{l}7302.92 \\
7305.13 \\
7326.55 \\
7342.85 \\
7353.95\end{array}$ & $\begin{array}{l}6 \\
1 \\
7 \\
1 \\
1\end{array}$ & $\begin{array}{l}\mathbf{A} \\
\mathbf{E} \\
\mathbf{A} \\
\mathbf{E} \\
\mathbf{E}\end{array}$ \\
\hline $\begin{array}{l}5552.02 \\
5567.78 \\
5573.10 \\
5573.75 \\
5602.02\end{array}$ & $\begin{array}{l}5 \\
4 \\
3 \\
3 \\
2, \mathrm{~h}, \mathrm{n} ?\end{array}$ & $\begin{array}{l}\text { B } \\
\text { C } \\
\text { B } \\
\text { C } \\
\text { B }\end{array}$ & $\begin{array}{l}6388.97 \\
6391.24 \\
6413.93 \\
6440.95 \\
6443.48\end{array}$ & $\begin{array}{l}1 \\
2 \\
3 \\
5 \\
3\end{array}$ & $\begin{array}{l}\text { B } \\
\text { B } \\
\text { B } \\
\text { B } \\
\text { B }\end{array}$ & $\begin{array}{l}7376.87 \\
7383.59 \\
7387.31 \\
7391.68 \\
7415.85\end{array}$ & $\begin{array}{l}1 \\
1 \\
1 \\
1 \\
2\end{array}$ & $\begin{array}{l}\mathbf{E} \\
\underset{\mathbf{E}}{\mathbf{E}} \\
\mathbf{E} \\
\mathbf{E}\end{array}$ \\
\hline $\begin{array}{l}5718.16 \\
5720.24 \\
5723.81 \\
5725.84 \\
5738.27\end{array}$ & $\begin{array}{l}3 \\
4 \\
2 \\
1 \\
3\end{array}$ & $\begin{array}{l}\text { B } \\
\text { B } \\
\text { B } \\
\text { B } \\
\text { B }\end{array}$ & $\begin{array}{l}6475.35 \\
6483.02 \\
6490.61 \\
6491.71 \\
6518.79\end{array}$ & $\begin{array}{l}2 \\
1 \\
2 \\
7 \\
2\end{array}$ & $\begin{array}{l}\text { B } \\
\text { B } \\
\text { C } \\
\text { A } \\
\text { A }\end{array}$ & $\begin{array}{l}7425.57 \\
7432.38 \\
7447.90 \\
7515.72 \\
7552.20\end{array}$ & $\begin{array}{l}1 \\
1 \\
1 \\
2 \\
1\end{array}$ & $\begin{array}{l}\mathbf{E} \\
\mathbf{E} \\
\mathbf{E} \\
\mathbf{C} \\
\mathbf{C}\end{array}$ \\
\hline $\begin{array}{l}5741.46 \\
5748.54 \\
5780.15 \\
5792.46 \\
5805.90\end{array}$ & $\begin{array}{l}1 \\
1 \\
3 \\
1 \\
1\end{array}$ & $\begin{array}{l}\mathrm{E} \\
\mathrm{B} \\
\mathrm{B} \\
\mathrm{C} \\
\mathrm{B}\end{array}$ & $\begin{array}{l}6519.38 \\
6526.52 \\
6533.50 \\
6534.08 \\
6552.01\end{array}$ & $\begin{array}{l}3 \\
2, \text { g coin? } \\
2 \\
2 \\
1\end{array}$ & $\begin{array}{l}\mathrm{A} \\
\mathrm{B} \\
\mathrm{B} \\
\mathrm{A} \\
\mathrm{B}\end{array}$ & $\begin{array}{l}7603.39 \\
7646.34 \\
7651.91 \\
7656.24 \\
7668.00\end{array}$ & $\begin{array}{l}2 \\
3, \mathrm{~b}, \mathrm{~h} \\
3, \mathrm{~b}, \mathrm{~h} \\
2, \mathrm{~b}, \mathrm{~h} \\
2\end{array}$ & $\begin{array}{l}\text { C } \\
\text { C } \\
\text { D } \\
\text { D } \\
\text { D }\end{array}$ \\
\hline $\begin{array}{l}5813.01 \\
5816.84 \\
5822.55 \\
5835.38 \\
5848.98\end{array}$ & $\begin{array}{l}1 \\
3 \\
1 \\
1 \\
3 \\
3\end{array}$ & $\begin{array}{l}\mathrm{C} \\
\mathrm{B} \\
\mathrm{C} \\
\mathrm{B} \\
\mathrm{B}\end{array}$ & $\begin{array}{l}6558.01 \\
6569.30 \\
6570.82 \\
6598.31 \\
6605.58\end{array}$ & $\begin{array}{l}1, \mathrm{Mn} ? \\
1 \\
3 \\
2 \\
4\end{array}$ & $\begin{array}{l}\text { B } \\
\text { C } \\
\text { C } \\
\text { C } \\
\text { B }\end{array}$ & $\begin{array}{l}7670.46 \\
7677.80 \\
7680.20 \\
7706.64 \\
7710.21\end{array}$ & $\begin{array}{l}2 \\
1, b, h \\
5 \\
2 \\
5\end{array}$ & $\begin{array}{l}\mathrm{D} \\
\mathrm{D} \\
\mathrm{A} \\
\mathrm{D} \\
\mathrm{B}\end{array}$ \\
\hline $\begin{array}{l}5868.18 \\
5879.18 \\
5882.50 \\
5888.12 \\
6013.50\end{array}$ & $\begin{array}{l}1, p ? \\
2, p ? \\
2, b, p ? \\
1, p ? \\
10\end{array}$ & $\begin{array}{l}\mathrm{B} \\
\mathrm{B} \\
\mathrm{A} \\
\mathrm{E} \\
\mathrm{B}\end{array}$ & $\begin{array}{l}6691.59 \\
6709.02 \\
6833.90 \\
6863.08 \\
6867.18\end{array}$ & $\begin{array}{l}2 \\
1 \\
2 \\
2 \\
2\end{array}$ & $\begin{array}{l}\mathrm{B} \\
\mathrm{B} \\
\mathrm{A} \\
\mathrm{C} \\
\mathrm{C}\end{array}$ & $\begin{array}{l}7712.42 \\
7721.55 \\
7727.22 \\
7733.23 \\
7734.49\end{array}$ & $\begin{array}{l}5, \mathbf{h} \\
1 \\
1 \\
4, \mathbf{h} \\
2\end{array}$ & $\begin{array}{l}\text { B } \\
\text { B } \\
\text { D } \\
\mathbf{A} \\
\mathbf{B}\end{array}$ \\
\hline $\begin{array}{l}6016.65 \\
6021.81 \\
6041.76 \\
6047.28 \\
6057.11\end{array}$ & $\begin{array}{l}10 \\
10 \\
2 \\
1, h \\
2\end{array}$ & $\begin{array}{l}\mathrm{B} \\
\mathrm{B} \\
\mathrm{B} \\
\mathrm{E} \\
\mathrm{B}\end{array}$ & $\begin{array}{l}6887.73 \\
6931.27 \\
6942.57 \\
6989.85 \\
7012.22\end{array}$ & $\begin{array}{l}3 \\
3 \\
5 \\
4 \\
2, \mathrm{~h}\end{array}$ & $\begin{array}{l}\text { B } \\
\text { B } \\
\text { A } \\
\text { A } \\
\text { B }\end{array}$ & $\begin{array}{l}7737.13 \\
7752.78 \\
7755.07 \\
7764.75 \\
7790.91\end{array}$ & $\begin{array}{l}1 \\
2 \\
3 \\
5 \\
2\end{array}$ & $\begin{array}{l}\text { B } \\
\text { D } \\
\text { D } \\
\text { A } \\
\text { D }\end{array}$ \\
\hline $\begin{array}{l}6078.41 \\
6090.14 \\
6095.68 \\
6109.06 \\
6114.33\end{array}$ & $\begin{array}{l}3, \mathrm{Mn} ? \\
2 \\
1 \\
3 \\
2, \mathrm{Mn} ?\end{array}$ & $\begin{array}{l}\text { B } \\
\text { B } \\
\text { C } \\
\text { B } \\
\text { C }\end{array}$ & $\begin{array}{l}7052.39 \\
7069.86 \\
7151.33 \\
7158.14 \\
7171.21\end{array}$ & $\begin{array}{l}1 \\
4 \\
8 \\
2 \\
1\end{array}$ & $\begin{array}{l}\mathrm{E} \\
\mathrm{A} \\
\mathrm{A} \\
\mathrm{C} \\
\mathbf{E}\end{array}$ & $\begin{array}{l}7805.99 \\
7816.59 \\
7821.25 \\
7834.32 \\
7854.34\end{array}$ & $\begin{array}{l}2 \\
2 \\
2 \\
2, \mathrm{~b} \\
1\end{array}$ & $\begin{array}{l}\text { B } \\
\text { B } \\
\text { B } \\
\text { B } \\
\text { E }\end{array}$ \\
\hline $\begin{array}{l}6176.06 \\
6177.16 \\
6177.52 \\
6178.51 \\
6223.78\end{array}$ & $\begin{array}{l}1 \\
1 \\
1 \\
1 \\
1\end{array}$ & $\begin{array}{l}\mathrm{E} \\
\mathrm{E} \\
\mathbf{E} \\
\mathrm{E} \\
\mathrm{E}\end{array}$ & $\begin{array}{l}7174.46 \\
7184.29 \\
7203.00 \\
7209.58 \\
7211.93\end{array}$ & $\begin{array}{l}2 \\
5 \\
1 \\
2 \\
2, \mathrm{~h}\end{array}$ & $\begin{array}{l}\mathrm{E} \\
\mathrm{A} \\
\mathrm{E} \\
\mathrm{E} \\
\mathrm{E}\end{array}$ & $\begin{array}{l}7916.31 \\
7920.44 \\
7928.45 \\
7942.87 \\
7948.06\end{array}$ & $\begin{array}{l}1 \\
1 \\
1 \\
2 \\
1\end{array}$ & $\begin{array}{l}\mathbf{E} \\
\mathbf{E} \\
\mathbf{E} \\
\mathbf{C} \\
\mathbf{C}\end{array}$ \\
\hline $\begin{array}{l}6237.70 \\
6265.67 \\
6268.48 \\
6315.05 \\
6344.11\end{array}$ & $\begin{array}{l}1, \mathrm{~h} \\
2 \\
1, \mathrm{~h} \\
2 \\
3\end{array}$ & $\begin{array}{l}\mathrm{E} \\
\mathrm{C} \\
\mathrm{E} \\
\mathrm{C} \\
\mathrm{B}\end{array}$ & $\begin{array}{l}7247.83 \\
7255.24 \\
7275.73 \\
7283.80 \\
7301.44\end{array}$ & $\begin{array}{l}5 \\
1, h, d ? \\
2 \\
6 \\
1\end{array}$ & $\begin{array}{l}\mathbf{B} \\
\mathrm{E} \\
\mathrm{E} \\
\mathrm{A} \\
\mathrm{E}\end{array}$ & $\begin{array}{l}8079.42 \\
8105.89 \\
8110.47 \\
8160.84 \\
8212.43\end{array}$ & $\begin{array}{l}1 \\
1 \\
1 \\
1 \\
2\end{array}$ & $\begin{array}{l}\mathbf{E} \\
\mathbf{E} \\
\mathbf{E} \\
\mathbf{E} \\
\mathbf{B}\end{array}$ \\
\hline
\end{tabular}


TABLE 4-Continued

\begin{tabular}{|c|c|c|c|c|c|c|c|c|}
\hline$\lambda$ I. A. & Notes & $\begin{array}{c}\text { Probable } \\
\text { error }\end{array}$ & $\lambda$ I. A. & Notes & $\begin{array}{c}\text { Probabie } \\
\text { error. }\end{array}$ & $\lambda$ I. A. & Notes & $\begin{array}{c}\text { Probable } \\
\text { error }\end{array}$ \\
\hline $\begin{array}{l}8227.04 \\
8280.73 \\
8287.76 \\
8373.97 \\
8380.76\end{array}$ & $\begin{array}{l}1 \\
1 \\
1 \\
1 \\
1\end{array}$ & $\begin{array}{l}\mathrm{E} \\
\mathrm{E} \\
\mathrm{E} \\
\mathrm{E}\end{array}$ & $\begin{array}{l}8600.39 \\
8602.07 \\
8654.60 \\
8659.35 \\
8664.66\end{array}$ & $\begin{array}{l}1 \\
1 \\
2 \\
2 \\
1, b, h\end{array}$ & $\begin{array}{l}\mathrm{E} \\
\mathrm{E} \\
\mathrm{B} \\
\mathrm{C} \\
\mathrm{E}\end{array}$ & $\begin{array}{l}8734.64 \\
8737.29 \\
8740.91 \\
8767.94 \\
8796.71\end{array}$ & $\begin{array}{l}1 \text { Mn? } \\
2 \\
3 \\
1 \\
1\end{array}$ & $\begin{array}{l}\mathbf{B} \\
\mathbf{B} \\
\mathbf{B} \\
\mathrm{E} \\
\mathrm{E}\end{array}$ \\
\hline $\begin{array}{l}8395.82 \\
8408.36 \\
8409.86 \\
8417.37 \\
8421.31\end{array}$ & $\begin{array}{l}1, b, b \\
1 \\
1 \\
1 \\
1\end{array}$ & $\begin{array}{l}\underset{\mathbf{E}}{\mathrm{E}} \\
\mathrm{C} \\
\mathbf{E} \\
\mathbf{E}\end{array}$ & $\begin{array}{l}8666.31 \\
8670.85 \\
8672.08 \\
8674.01 \\
8699.13\end{array}$ & $\begin{array}{l}1, \mathrm{~b}, \mathrm{~h} \\
2 \\
2 \\
2, \mathrm{~h} \\
2\end{array}$ & $\begin{array}{l}\mathbf{E} \\
\mathbf{B} \\
\mathbf{B} \\
\mathbf{B} \\
\mathbf{B}\end{array}$ & $\begin{array}{l}8798.76 \\
8929.66 \\
8933.03 \\
9084.34\end{array}$ & $\begin{array}{l}1, \mathrm{~h} \\
1 \\
1 \\
1\end{array}$ & $\begin{array}{l}E \\
E \\
E \\
C\end{array}$ \\
\hline $\begin{array}{l}8425.54 \\
8431.14 \\
8435.19 \\
8521.54 \\
8558.52\end{array}$ & $\begin{array}{l}1 \\
1, b \\
1 \\
1 \\
1\end{array}$ & $\begin{array}{l}\mathrm{E} \\
\mathrm{D} \\
\mathrm{E} \\
\mathrm{E} \\
\mathrm{E}\end{array}$ & $\begin{array}{l}8701.04 \\
8703.73 \\
8710.14 \\
8717.31 \\
8731.76\end{array}$ & $\begin{array}{l}2 \\
3 \\
1 \\
1 \\
1\end{array}$ & $\begin{array}{l}\mathrm{B} \\
\mathrm{B} \\
\mathrm{E} \\
\mathrm{E} \\
\mathrm{E}\end{array}$ & $\begin{array}{l}9172.13 \\
9243.21 \\
9576.02\end{array}$ & $\begin{array}{l}1 \\
1 \\
1 \\
1\end{array}$ & $\begin{array}{l}\mathrm{C} \\
\mathrm{C} \\
\mathrm{E}\end{array}$ \\
\hline
\end{tabular}

\section{MOLYBDENUM}

Table 5 contains 545 lines in the arc spectrum of molybdenum measured between the limits $5500 \mathrm{~A}$ and $9800 \mathrm{~A}$. The spectrum of this element is characterized by a very large number of faint lines and a relatively small number of stronger lines. The measurements were rather difficult because of the strong background of continuous spectrum, probably due to the incandescent oxide that is formed, which is present on all the plates. It is hoped that the table is free from the lines of impurities, calcium and sodium being the only ones recognized thus far.

Our measurements are in fair agreement with those made by Weigand, ${ }^{16}$ who measured the arc spectrum of molybdenum between the wave-length limits $4647 \mathrm{~A}$ and $7134 \mathrm{~A}$.

Paulson ${ }^{17}$ has given a number of pairs of lines with constant frequency differences. Additional groups found among the lines of Table 5 are:

\begin{tabular}{|c|r|r|r|}
\hline$\lambda$ & & \multicolumn{1}{|c|}{$\nu$} & \multicolumn{1}{|c|}{$\Delta \nu$} \\
\hline 5506.54 & 9 & 18160.23 & \\
5533.06 & 10 & 18073.18 & 87.05 \\
5570.57 & 7 & 17951.49 & 121.69 \\
5650.15 & 4 & 17698.64 & \\
5689.22 & 7 & 17577.10 & 121.54 \\
5722.78 & 5 & 17474.02 & \\
5751.42 & 6 & 17387.01 & 87.01 \\
5791.88 & 6 & 17265.55 & 121.46 \\
5858.28 & 7 & 17069.86 & \\
5888.32 & 6 & 16982.77 & 87.09 \\
8328.43 & 5 & 12007.07 & \\
8389.28 & 6 & 11919.98 & 87.09 \\
7242.54 & 7 & 13807.31 & \\
7485.73 & 7 & 13358.75 & 448.56 \\
\hline
\end{tabular}

${ }^{16}$ Zeit. für Wiss. Phot., 11, p. 26r; r9I2.

${ }^{17}$ Paulson, Dissertation, Lund, I9I4; also Astrophysical Journal, 40, p. 302; 19I4. 
TABLE 5.-Arc Spectrum of Molybdenum

\begin{tabular}{|c|c|c|c|c|c|c|c|c|}
\hline$\lambda$ I. A. & Notes & $\begin{array}{c}\text { Probable } \\
\text { error }\end{array}$ & $\lambda$ I. A. & Notes & $\begin{array}{c}\text { Probable } \\
\text { error }\end{array}$ & $\lambda$ I. A. & Notes & $\begin{array}{c}\text { Probable } \\
\text { error }\end{array}$ \\
\hline $\begin{array}{l}5501.76 \\
5503.60 \\
5506.54 \\
5511.51 \\
5513.89\end{array}$ & $\begin{array}{l}3 \\
2 \\
9 \\
2 \\
1\end{array}$ & $\begin{array}{l}\mathbf{C} \\
\mathbf{E} \\
\mathrm{B} \\
\mathrm{E} \\
\mathrm{E}\end{array}$ & $\begin{array}{l}5741.74 \\
5746.85 \\
5747.61 \\
5751.42 \\
5765.33\end{array}$ & $\begin{array}{l}2 \\
1 \\
1 \\
6 \\
1\end{array}$ & $\begin{array}{l}\mathbf{B} \\
\mathbf{E} \\
\mathbf{C} \\
\mathbf{B} \\
\mathbf{E}\end{array}$ & $\begin{array}{l}5965.52 \\
5968.47 \\
5970.50 \\
5974.25 \\
5982.88\end{array}$ & $\begin{array}{l}1 \\
1 \\
1 \\
2 \\
2\end{array}$ & $\begin{array}{l}\mathbf{C} \\
\mathbf{C} \\
\mathbf{E} \\
\mathbf{B} \\
\mathbf{B}\end{array}$ \\
\hline $\begin{array}{l}5517.54 \\
5520.24 \\
5520.76 \\
5526.84 \\
5529.32\end{array}$ & $\begin{array}{l}1 \\
2 \\
2 \\
3 \\
1 \\
1\end{array}$ & $\begin{array}{l}\mathbf{E} \\
\mathbf{E} \\
\mathbf{E} \\
\mathbf{C} \\
\mathbf{E}\end{array}$ & $\begin{array}{l}5766.59 \\
5769.78 \\
5771.06 \\
5774.47 \\
5775.97\end{array}$ & $\begin{array}{l}1 \\
1 \\
1 \\
2 \\
1\end{array}$ & $\begin{array}{l}\mathbf{E} \\
\mathbf{B} \\
\mathbf{B} \\
\mathbf{C} \\
\mathbf{E}\end{array}$ & $\begin{array}{l}5988.12 \\
5989.57 \\
5990.01 \\
5991.34 \\
6000.88\end{array}$ & $\begin{array}{l}2 \\
2 \\
1 \\
1 \\
1\end{array}$ & $\begin{array}{l}\mathbf{C} \\
\mathbf{C} \\
\mathbf{E} \\
\mathbf{C} \\
\mathbf{E}\end{array}$ \\
\hline $\begin{array}{l}5533.06 \\
5537.85 \\
5539.48 \\
5541.73 \\
5543.14\end{array}$ & $\begin{array}{l}10 \\
1 \\
2 \\
1 \\
2\end{array}$ & $\begin{array}{l}\mathbf{C} \\
\mathbf{E} \\
\mathbf{C} \\
\mathbf{E} \\
\mathbf{B}\end{array}$ & $\begin{array}{l}5778.13 \\
5779.30 \\
5780.07 \\
5780.71 \\
5783.33\end{array}$ & $\begin{array}{l}1 \\
2 \\
1 \\
1 \\
2\end{array}$ & $\begin{array}{l}\mathbf{B} \\
\mathbf{B} \\
\mathbf{C} \\
\mathbf{E} \\
\mathbf{A}\end{array}$ & $\begin{array}{l}6003.99 \\
6011.29 \\
6012.26 \\
6013.09 \\
6025.51\end{array}$ & $\begin{array}{l}1 \\
1 \\
1 \\
1 \\
2\end{array}$ & $\begin{array}{l}\mathbf{E} \\
\mathbf{C} \\
\mathbf{E} \\
\mathbf{E} \\
\mathbf{B}\end{array}$ \\
\hline $\begin{array}{l}5544.57 \\
5552.13 \\
5556.56 \\
5562.56 \\
5564.12\end{array}$ & $\begin{array}{l}2 \\
2, \mathrm{~h} \\
3, \mathrm{~d} \\
\frac{1}{2}\end{array}$ & $\begin{array}{l}\mathbf{C} \\
\mathbf{C} \\
\mathrm{B} \\
\mathrm{E} \\
\mathbf{B}\end{array}$ & $\begin{array}{l}5785.69 \\
5791.88 \\
5795.74 \\
5800.41 \\
5801.45\end{array}$ & $\begin{array}{l}1 \\
6 \\
2 \\
2 \\
1\end{array}$ & $\begin{array}{l}\mathbf{B} \\
\mathbf{B} \\
\mathbf{B} \\
\mathbf{B} \\
\mathbf{B}\end{array}$ & $\begin{array}{l}6027.26 \\
6030.65 \\
6036.40 \\
6039.72 \\
6047.89\end{array}$ & $\begin{array}{l}1 \\
9 \\
1 \\
1 \\
2\end{array}$ & $\begin{array}{l}\mathbf{B} \\
\mathbf{B} \\
\mathbf{E} \\
\mathbf{C} \\
\mathbf{B}\end{array}$ \\
\hline $\begin{array}{l}5566.76 \\
5568.70 \\
5570.57 \\
5575.21 \\
5580.19\end{array}$ & $\begin{array}{l}1 \\
2 \\
7 \\
2 \\
1\end{array}$ & $\begin{array}{l}\mathbf{E} \\
\mathbf{C} \\
\mathbf{B} \\
\mathbf{B} \\
\mathbf{E}\end{array}$ & $\begin{array}{l}5802.70 \\
5803.99 \\
5806.29 \\
5806.69 \\
5808.14\end{array}$ & $\begin{array}{l}2 \\
1 \\
1 \\
1 \\
1\end{array}$ & $\begin{array}{l}\mathbf{B} \\
\mathbf{B} \\
\mathbf{C} \\
\mathbf{B} \\
\mathbf{B}\end{array}$ & $\begin{array}{l}6050.96 \\
6054.84 \\
6058.01 \\
6063.90 \\
6075.56\end{array}$ & $\begin{array}{l}1 \\
2 \\
1 \\
1 \\
1\end{array}$ & $\begin{array}{l}\text { C } \\
\text { A } \\
\text { A } \\
\text { B } \\
\text { C }\end{array}$ \\
\hline $\begin{array}{l}5591.67 \\
5594.86 \\
5596.39 \\
5608.69 \\
5609.46\end{array}$ & $\begin{array}{l}2 \\
1 \\
1 \\
2 \\
2, \mathrm{~d}\end{array}$ & $\begin{array}{l}\mathbf{B} \\
\mathbf{C} \\
\mathbf{C} \\
\mathbf{B} \\
\mathrm{B}\end{array}$ & $\begin{array}{l}5809.13 \\
5812.51 \\
5813.93 \\
5815.62 \\
5820.68\end{array}$ & $\begin{array}{l}1 \\
1 \\
2 \\
2 \\
1\end{array}$ & $\begin{array}{l}\mathbf{E} \\
\mathbf{C} \\
\mathrm{C} \\
\mathrm{B} \\
\mathrm{B}\end{array}$ & $\begin{array}{l}6079.58 \\
6081.25 \\
608.72 \\
6096.20 \\
6101.87\end{array}$ & $\begin{array}{l}2 \\
2 \\
1 \\
1 \\
3\end{array}$ & $\begin{array}{l}\text { C } \\
\text { B } \\
\text { B } \\
\text { C } \\
\text { C }\end{array}$ \\
\hline $\begin{array}{l}5611.00 \\
5613.15 \\
5618.50 \\
5619.56 \\
5627.19\end{array}$ & $\begin{array}{l}3 \\
2 \\
2 \\
2 \\
1\end{array}$ & $\begin{array}{l}\mathbf{B} \\
\mathbf{B} \\
\mathbf{B} \\
\mathbf{B} \\
\mathbf{E}\end{array}$ & $\begin{array}{l}5825.19 \\
5827.02 \\
5835.63 \\
5839.98 \\
5841.76\end{array}$ & $\begin{array}{l}3 \\
1 \\
1 \\
1 \\
1\end{array}$ & $\begin{array}{l}\mathbf{B} \\
\mathbf{E} \\
\mathbf{B} \\
\mathbf{A} \\
\mathbf{E}\end{array}$ & $\begin{array}{l}6107.23 \\
6110.61 \\
6111.87 \\
6119.00 \\
6120.61\end{array}$ & $b, d ?$ & $\begin{array}{l}\mathbf{E} \\
\mathbf{B} \\
\mathbf{E} \\
\mathbf{E} \\
\mathbf{C}\end{array}$ \\
\hline $\begin{array}{l}5632.48 \\
5634.91 \\
5650.15 \\
5651.44 \\
5652.07\end{array}$ & $\begin{array}{l}4 \\
3 \\
4 \\
1 \\
2\end{array}$ & $\begin{array}{l}\mathbf{B} \\
\mathbf{B} \\
\mathrm{B} \\
\mathbf{C} \\
\mathbf{C}\end{array}$ & $\begin{array}{l}5848.82 \\
5849.76 \\
5851.52 \\
5554.36 \\
5858.28\end{array}$ & $\begin{array}{l}2 \\
3 \\
3 \\
1 \\
7\end{array}$ & $\begin{array}{l}\mathbf{B} \\
\mathbf{B} \\
\mathbf{B} \\
\mathbf{E} \\
\mathbf{A}\end{array}$ & $\begin{array}{l}6123.69 \\
6125.41 \\
6128.19 \\
6130.77 \\
6138.63\end{array}$ & $\begin{array}{l}2 \\
1 \\
2 \\
2 \\
1\end{array}$ & $\begin{array}{l}\mathbf{B} \\
\mathbf{B} \\
\mathbf{B} \\
\mathbf{B} \\
\mathbf{E}\end{array}$ \\
\hline $\begin{array}{l}5655.49 \\
5662.56 \\
5664.44 \\
5667.34 \\
5672.14\end{array}$ & $\begin{array}{l}1 \\
1 \\
2 \\
2 \\
2 \\
2\end{array}$ & $\begin{array}{l}\mathrm{E} \\
\mathrm{E} \\
\mathrm{B} \\
\mathrm{B} \\
\mathrm{B}\end{array}$ & $\begin{array}{l}5861.41 \\
5869.32 \\
5871.54 \\
5874.23 \\
5876.55\end{array}$ & $\begin{array}{l}2 \\
4 \\
1 \\
1 \\
2\end{array}$ & $\begin{array}{l}\text { B } \\
\text { B } \\
\text { C } \\
\text { B } \\
\text { A }\end{array}$ & $\begin{array}{l}6148.56 \\
6158.43 \\
6169.11 \\
6170.79 \\
6175.56\end{array}$ & $\begin{array}{l}\frac{1}{2}, \mathrm{~b}, \mathrm{~b} \\
1 \\
1 \\
1 \\
1\end{array}$ & $\begin{array}{l}\mathbf{C} \\
\mathbf{C} \\
\mathbf{C} \\
\mathbf{B} \\
\mathbf{C}\end{array}$ \\
\hline $\begin{array}{l}5673.60 \\
5674.55 \\
5677.93 \\
5687.67 \\
5689.22\end{array}$ & $\begin{array}{l}2 \\
2 \\
2 \\
1 \\
7\end{array}$ & $\begin{array}{l}\mathbf{B} \\
\mathbf{B} \\
\mathbf{B} \\
\mathbf{B} \\
\mathbf{A}\end{array}$ & $\begin{array}{l}5878.16 \\
5881.50 \\
588.78 \\
5884.33 \\
5888.32\end{array}$ & $\begin{array}{l}1 \\
1 \\
1 \\
2 \\
6\end{array}$ & $\begin{array}{l}\mathrm{A} \\
\mathrm{A} \\
\mathrm{B} \\
\mathrm{B} \\
\mathrm{B}\end{array}$ & $\begin{array}{l}6189.01 \\
6193.20 \\
6197.66 \\
6206.18 \\
6208.31\end{array}$ & $\begin{array}{l}2 \\
1 \\
2 \\
2 \\
1\end{array}$ & $\begin{array}{l}\mathrm{B} \\
\mathrm{B} \\
\mathrm{C} \\
\mathrm{E} \\
\mathrm{E}\end{array}$ \\
\hline $\begin{array}{l}5694.43 \\
5696.02 \\
5698.26 \\
5699.33 \\
5702.15\end{array}$ & $\begin{array}{l}1 \\
1 \\
1 \\
2 \\
1\end{array}$ & $\begin{array}{l}\text { C } \\
\text { B } \\
\text { B } \\
\text { A } \\
\text { A }\end{array}$ & $\begin{array}{l}5891.65 \\
5892.30 \\
5893.41 \\
5897.84 \\
5898.78\end{array}$ & $\begin{array}{l}2 \\
2 \\
3 \\
1 \\
2\end{array}$ & $\begin{array}{l}C \\
C \\
B \\
\text { E } \\
B\end{array}$ & $\begin{array}{l}6209.61 \\
6213.28 \\
6214.66 \\
6217.93 \\
6228.63\end{array}$ & $\begin{array}{l}1 \\
1 \\
1 \\
2 \\
1, \mathrm{~b}\end{array}$ & $\begin{array}{l}\mathbf{B} \\
\mathbf{C} \\
\mathbf{C} \\
\mathbf{B} \\
\mathbf{C}\end{array}$ \\
\hline $\begin{array}{l}5705.74 \\
5708.03 \\
5711.82 \\
5715.17 \\
5716.07\end{array}$ & $\begin{array}{l}3 \\
1 \\
2 \\
1 \\
1 \\
1\end{array}$ & $\begin{array}{l}\mathrm{A} \\
\mathrm{A} \\
\mathrm{B} \\
\mathrm{E} \\
\mathrm{E}\end{array}$ & $\begin{array}{l}5899.72 \\
5901.46 \\
5912.11 \\
5914.30 \\
5917.55\end{array}$ & $\begin{array}{l}1 \\
3 \\
1 \\
1 \\
1\end{array}$ & $\begin{array}{l}\mathrm{B} \\
\mathrm{B} \\
\mathrm{B} \\
\mathrm{B} \\
\mathrm{E}\end{array}$ & $\begin{array}{l}6244.80 \\
6264.27 \\
6265.87 \\
6281.89 \\
6286.63\end{array}$ & $\begin{array}{l}1 \\
1 \\
2 \\
1 \\
1\end{array}$ & $\begin{array}{l}\mathbf{B} \\
\text { B } \\
\text { B } \\
\text { C } \\
\text { B }\end{array}$ \\
\hline $\begin{array}{l}5719.38 \\
5720.27 \\
5722.78 \\
5728.77 \\
5729.75\end{array}$ & $\begin{array}{l}1 \\
1 \\
5 \\
2 \\
2\end{array}$ & $\begin{array}{l}\mathbf{E} \\
\mathrm{E} \\
\mathrm{A} \\
\mathrm{B} \\
\mathrm{A}\end{array}$ & $\begin{array}{l}5923.81 \\
5926.33 \\
5928.81 \\
5933.39 \\
5937.89\end{array}$ & $\begin{array}{l}1 \\
4 \\
7 \\
1 \\
2\end{array}$ & $\begin{array}{l}\mathrm{C} \\
\mathrm{B} \\
\mathrm{B} \\
\mathrm{E} \\
\mathrm{B}\end{array}$ & $\begin{array}{l}6290.74 \\
6301.76 \\
6323.54 \\
6339.90 \\
6357.22\end{array}$ & $\begin{array}{l}2 \\
2 \\
1 \\
1 \\
4\end{array}$ & $\begin{array}{l}\text { B } \\
\text { B } \\
\text { C } \\
\text { B } \\
\text { B }\end{array}$ \\
\hline $\begin{array}{l}5734.07 \\
5735.34 \\
5736.55 \\
5738.12 \\
5739.62\end{array}$ & $\begin{array}{l}2 \\
1 \\
1 \\
1 \\
1 \\
1\end{array}$ & $\begin{array}{l}B \\
\text { E } \\
\text { E } \\
\text { E } \\
\text { B }\end{array}$ & $\begin{array}{l}5943.60 \\
5949.14 \\
5951.97 \\
5957.68 \\
5962.72\end{array}$ & $\begin{array}{l}1 \\
1 \\
2 \\
1 \\
1, \mathrm{~b}\end{array}$ & $\begin{array}{l}\mathrm{C} \\
\mathrm{B} \\
\mathrm{B} \\
\mathrm{B} \\
\mathrm{C}\end{array}$ & $\begin{array}{l}6358.58 \\
6372.27 \\
6383.70 \\
6389.03 \\
6391.03\end{array}$ & $\begin{array}{l}1 \\
1 \\
1 \\
2 \\
2\end{array}$ & $\begin{array}{l}\mathrm{E} \\
\mathbf{C} \\
\mathbf{E} \\
\mathbf{E} \\
\mathbf{C}\end{array}$ \\
\hline
\end{tabular}


TABLE 5-Continued

\begin{tabular}{|c|c|c|c|c|c|c|c|c|}
\hline$\lambda$ I. A. & Notes & $\begin{array}{c}\text { Probable } \\
\text { error }\end{array}$ & $\lambda$ I. A. & Notes & $\begin{array}{c}\text { Probable } \\
\text { error }\end{array}$ & $\lambda$ I. A. & Notes & $\begin{array}{c}\text { Probable } \\
\text { error }\end{array}$ \\
\hline $\begin{array}{l}6401.08 \\
6404.45 \\
6406.56 \\
6409.10 \\
6412.43\end{array}$ & $\begin{array}{l}2 \\
1, \mathrm{~h} \\
1, \mathrm{~b}, \mathrm{~h} \\
3 \\
2, \mathrm{~b}\end{array}$ & $\begin{array}{l}\mathbf{B} \\
\mathbf{E} \\
\mathbf{B} \\
\stackrel{\mathbf{B}}{\mathbf{C}} \\
\mathbf{C}\end{array}$ & $\begin{array}{l}6724.89 \\
6728.02 \\
6733.97 \\
6746.24 \\
6753.99\end{array}$ & $\begin{array}{l}1 \\
2 \\
6 \\
5 \\
3\end{array}$ & $\begin{array}{l}\mathbf{E} \\
\mathbf{A} \\
\mathbf{A} \\
\mathbf{A} \\
\mathbf{A}\end{array}$ & $\begin{array}{l}6999.10 \\
6999.91 \\
7001.62 \\
7011.78 \\
7013.95\end{array}$ & $\begin{array}{l}2 \\
1 \\
3, \mathrm{Ni} \text { ? } \\
1\end{array}$ & $\begin{array}{l}\mathbf{A} \\
\mathbf{A} \\
\mathbf{A} \\
\mathbf{B} \\
\mathbf{A}\end{array}$ \\
\hline $\begin{array}{l}6412.92 \\
6414.23 \\
6418.95 \\
6419.89 \\
6424.37\end{array}$ & $\begin{array}{l}1 \\
1 \\
1 \\
1 \\
5\end{array}$ & $\begin{array}{l}\mathrm{E} \\
\mathrm{E} \\
\mathrm{D} \\
\mathbf{C} \\
\mathrm{B}\end{array}$ & $\begin{array}{l}6759.62 \\
6761.58 \\
6763.53 \\
6764.94 \\
6765.70\end{array}$ & $\begin{array}{l}1 \\
1 \\
2 \\
1 \\
1\end{array}$ & $\begin{array}{l}\mathbf{E} \\
\mathbf{E} \\
\mathbf{A} \\
\mathbf{A} \\
\mathbf{A}\end{array}$ & $\begin{array}{l}7016.46 \\
7018.42 \\
7019.61 \\
7025.32 \\
7032.30\end{array}$ & $\begin{array}{l}2 \\
1 \\
1 \\
2 \\
1\end{array}$ & $\begin{array}{l}\mathbf{A} \\
\mathbf{A} \\
\mathbf{A} \\
\mathbf{A} \\
\mathbf{B}\end{array}$ \\
\hline $\begin{array}{l}6429.02 \\
6439.80 \\
6446.34 \\
6453.30 \\
6461.93\end{array}$ & $\begin{array}{l}2 \\
2 \\
3 \\
2 \\
2\end{array}$ & $\begin{array}{l}\text { B } \\
\text { E } \\
\text { B } \\
\text { B } \\
\text { C }\end{array}$ & $\begin{array}{l}6767.94 \\
6771.22 \\
6773.36 \\
6774.67 \\
6778.63\end{array}$ & $\begin{array}{l}1 \\
1 \\
1 \\
1 \\
2\end{array}$ & $\begin{array}{l}\mathbf{C} \\
\mathbf{E} \\
\mathbf{E} \\
\mathbf{B} \\
\mathbf{A}\end{array}$ & $\begin{array}{l}7037.97 \\
7045.29 \\
7060.23 \\
7063.34 \\
7064.55\end{array}$ & $\begin{array}{l}3 \\
2 \\
3 \\
2 \\
1\end{array}$ & $\begin{array}{l}\mathbf{A} \\
\mathbf{B} \\
\mathbf{A} \\
\mathbf{A} \\
\mathbf{A}\end{array}$ \\
\hline $\begin{array}{l}6463.57 \\
6467.02 \\
6471.22 \\
6474.01 \\
6476.34\end{array}$ & $\begin{array}{l}1 \\
1 \\
3 \\
3 \\
1\end{array}$ & $\begin{array}{l}\mathbf{B} \\
\mathbf{B} \\
\mathbf{B} \\
\mathbf{B} \\
\mathbf{B}\end{array}$ & $\begin{array}{l}6787.96 \\
6789.01 \\
6792.40 \\
6794.56 \\
6799.94\end{array}$ & $\begin{array}{l}2 \\
2 \\
1 \\
1 \\
2\end{array}$ & $\begin{array}{l}\mathbf{A} \\
\mathbf{A} \\
\mathbf{C} \\
\mathbf{C} \\
\mathbf{A}\end{array}$ & $\begin{array}{l}7073.55 \\
7076.80 \\
7079.24 \\
7081.22 \\
7083.12\end{array}$ & $\begin{array}{l}1 \\
1 \\
1 \\
2 \\
1\end{array}$ & $\begin{array}{l}\mathbf{A} \\
\mathbf{B} \\
\mathbf{B} \\
\mathbf{A} \\
\mathbf{B}\end{array}$ \\
\hline $\begin{array}{l}6479.23 \\
6485.97 \\
6492.48 \\
6493.16 \\
6512.68\end{array}$ & $\begin{array}{l}\frac{1}{3}, b, h \\
2 \\
2 \\
1\end{array}$ & $\begin{array}{l}\mathbf{B} \\
\mathbf{B} \\
\mathbf{B} \\
\mathbf{B} \\
\mathbf{B}\end{array}$ & $\begin{array}{l}6802.62 \\
6812.04 \\
6822.03 \\
6825.63 \\
6829.05\end{array}$ & $\begin{array}{l}2 \\
2 \\
1, \mathrm{~h} \\
2 \\
3, \mathrm{~h}\end{array}$ & $\begin{array}{l}\mathbf{A} \\
\mathbf{A} \\
\mathbf{A} \\
\mathbf{A} \\
\mathbf{A}\end{array}$ & $\begin{array}{l}7088.78 \\
7098.18 \\
7102.65 \\
7105.59 \\
7109.87\end{array}$ & $\begin{array}{l}2, \mathrm{~h} \\
1 \\
2 \\
2 \\
8\end{array}$ & $\begin{array}{l}\mathbf{B} \\
\mathbf{B} \\
\mathbf{B} \\
\mathbf{A} \\
\mathbf{B}\end{array}$ \\
\hline $\begin{array}{l}6519.83 \\
6523.53 \\
6527.62 \\
6536.26 \\
6537.66\end{array}$ & $\begin{array}{l}3 \\
1 \\
1 \\
2 \\
2\end{array}$ & $\begin{array}{l}\mathbf{A} \\
\mathbf{B} \\
\mathbf{B} \\
\mathbf{B} \\
\mathbf{B}\end{array}$ & $\begin{array}{l}6834.17 \\
6838.89 \\
6848.93 \\
6852.80 \\
6854.22\end{array}$ & $\begin{array}{l}1 \\
4 \\
3 \\
1 \\
1, \mathrm{~h}\end{array}$ & $\begin{array}{l}\mathbf{C} \\
\mathbf{A} \\
\mathbf{A} \\
\mathbf{E} \\
\mathbf{B}\end{array}$ & $\begin{array}{l}7122.65 \\
7124.32 \\
7127.31 \\
7134.09 \\
7135.73\end{array}$ & $\begin{array}{l}3 \\
1 \\
1 \\
4 \\
1\end{array}$ & $\begin{array}{l}\mathbf{A} \\
\mathbf{A} \\
\mathbf{B} \\
\mathbf{A} \\
\mathbf{A}\end{array}$ \\
\hline $\begin{array}{l}6552.45 \\
6553.47 \\
6565.76 \\
6569.74 \\
6571.06\end{array}$ & $\begin{array}{l}2 \\
1 \\
\frac{1}{2}, b, h \\
1\end{array}$ & $\begin{array}{l}\mathrm{D} \\
\mathrm{D} \\
\mathrm{B} \\
\mathrm{B} \\
\mathbf{B}\end{array}$ & $\begin{array}{l}6859.40 \\
6863.70 \\
6865.46 \\
6867.96 \\
6874.66\end{array}$ & $\begin{array}{l}1, \mathbf{h} \\
1 \\
1 \\
1 \\
1\end{array}$ & $\begin{array}{l}\mathbf{B} \\
\mathbf{B} \\
\mathbf{B} \\
\mathbf{E} \\
\mathbf{E}\end{array}$ & $\begin{array}{l}7136.67 \\
7146.24 \\
7149.45 \\
7154.15 \\
7159.80\end{array}$ & $\begin{array}{l}1 \\
1 \\
1 \\
1 \\
2\end{array}$ & $\begin{array}{l}\mathbf{A} \\
\mathbf{A} \\
\mathbf{A} \\
\mathbf{A} \\
\mathbf{B}\end{array}$ \\
\hline $\begin{array}{l}6572.31 \\
6580.03 \\
6590.87 \\
6611.25 \\
6619.16\end{array}$ & $\begin{array}{l}1 \\
1 \\
2 \\
3 \\
8\end{array}$ & $\begin{array}{l}\mathrm{B} \\
\mathrm{B} \\
\mathrm{C} \\
\mathrm{A} \\
\mathrm{A}\end{array}$ & $\begin{array}{l}6883.84 \\
6886.34 \\
6889.13 \\
6892.37 \\
6898.02\end{array}$ & $\begin{array}{l}1 \\
4 \\
2, \mathrm{~b}, \mathrm{~h} \\
3 \\
2\end{array}$ & $\begin{array}{l}\mathbf{E} \\
\mathbf{A} \\
\mathbf{B} \\
\mathbf{A} \\
\mathbf{A}\end{array}$ & $\begin{array}{l}7171.85 \\
7182.76 \\
7186.54 \\
7195.34 \\
7198.28\end{array}$ & $\begin{array}{l}1, \mathrm{~h} \\
1 \\
1 \\
1 \\
1\end{array}$ & $\begin{array}{l}\mathbf{B} \\
\mathbf{B} \\
\mathbf{E} \\
\mathbf{B} \\
\mathbf{E}\end{array}$ \\
\hline $\begin{array}{l}6624.57 \\
6634.01 \\
6637.15 \\
6649.43 \\
6649.78\end{array}$ & $\begin{array}{l}2 \\
1 \\
2 \\
1 \\
1\end{array}$ & $\begin{array}{l}\mathbf{B} \\
\mathbf{B} \\
\mathbf{B} \\
\mathbf{B} \\
\mathbf{E}\end{array}$ & $\begin{array}{l}6899.03 \\
6900.55 \\
6904.73 \\
6908.23 \\
6910.98\end{array}$ & $\begin{array}{l}2 \\
1, \mathrm{~h} \\
2 \\
2 \\
1, \mathrm{~h}\end{array}$ & $\begin{array}{l}\mathbf{A} \\
\mathbf{B} \\
\mathbf{B} \\
\mathbf{A} \\
\mathbf{B}\end{array}$ & $\begin{array}{l}7203.58 \\
7216.98 \\
7218.50 \\
7219.27 \\
7228.31\end{array}$ & $\begin{array}{l}1 \\
1 \\
1 \\
1 \\
1, \mathbf{h}\end{array}$ & $\begin{array}{l}\mathrm{E} \\
\mathrm{A} \\
\mathrm{E} \\
\mathrm{E} \\
\mathrm{B}\end{array}$ \\
\hline $\begin{array}{l}6650.40 \\
6653.54 \\
6657.01 \\
6659.68 \\
6672.22\end{array}$ & $\begin{array}{l}6, \mathbf{h} \\
1 \\
1 \\
3 \\
1\end{array}$ & $\begin{array}{l}\mathbf{B} \\
\text { D } \\
\mathbf{B} \\
\mathbf{A} \\
\mathbf{D}\end{array}$ & $\begin{array}{l}6914.04 \\
6917.61 \\
6925.90 \\
6929.03 \\
6931.42\end{array}$ & $\begin{array}{l}5 \\
1 \\
2 \\
1 \\
2\end{array}$ & $\begin{array}{l}\mathbf{A} \\
\mathbf{B} \\
\mathbf{B} \\
\mathbf{B} \\
\mathbf{A}\end{array}$ & $\begin{array}{l}7240.48 \\
7242.54 \\
7244.56 \\
725.87 \\
7249.89\end{array}$ & $\begin{array}{l}1 \\
7 \\
1 \\
4 \\
1\end{array}$ & $\begin{array}{l}\mathbf{A} \\
\mathbf{A} \\
\mathbf{A} \\
\mathbf{A} \\
\mathbf{E}\end{array}$ \\
\hline $\begin{array}{l}6673.89 \\
6675.42 \\
6678.88 \\
6679.59 \\
6681.94\end{array}$ & $\begin{array}{l}1 \\
1 \\
1, \mathrm{~h} \\
1 \\
1\end{array}$ & $\begin{array}{l}\mathbf{B} \\
\mathbf{C} \\
\mathbf{C} \\
\mathbf{C} \\
\mathbf{B}\end{array}$ & $\begin{array}{l}6934.12 \\
6935.75 \\
6937.12 \\
6946.71 \\
6947.46\end{array}$ & $\begin{array}{l}2 \\
1 \\
1 \\
1 \\
2\end{array}$ & $\begin{array}{l}\mathbf{A} \\
\mathbf{B} \\
\mathbf{B} \\
\mathbf{B} \\
\mathbf{B}\end{array}$ & $\begin{array}{l}7267.62 \\
7277.21 \\
7281.51 \\
7295.03 \\
7300.19\end{array}$ & $\begin{array}{l}3 \\
1 \\
1 \\
1 \\
2\end{array}$ & $\begin{array}{l}\mathbf{A} \\
\mathbf{B} \\
\mathbf{A} \\
\mathbf{A} \\
\mathbf{B}\end{array}$ \\
\hline $\begin{array}{l}6683.26 \\
6585.54 \\
6687.88 \\
6690.50 \\
6691.04\end{array}$ & $\begin{array}{l}1 \\
1 \\
2 \\
4 \\
2\end{array}$ & $\begin{array}{l}\text { B } \\
\mathbf{B} \\
\mathbf{A} \\
\mathbf{A} \\
\mathbf{B}\end{array}$ & $\begin{array}{l}6953.80 \\
6957.08 \\
6959.52 \\
6960.66 \\
6961.53\end{array}$ & $\begin{array}{l}2 \\
1 \\
1 \\
2 \\
2\end{array}$ & $\begin{array}{l}\mathbf{B} \\
\mathbf{B} \\
\mathbf{B} \\
\mathbf{B} \\
\mathbf{B}\end{array}$ & $\begin{array}{l}7311.11 \\
7313.91 \\
7315.36 \\
7322.53 \\
7325.37\end{array}$ & $\begin{array}{l}1 \\
1, \mathrm{~h} \\
1, \mathrm{~h} \\
2, \mathrm{v}, \mathrm{d} \\
1\end{array}$ & $\begin{array}{l}\text { B } \\
\text { B } \\
\text { C } \\
\text { B } \\
\text { C }\end{array}$ \\
\hline $\begin{array}{l}6692.49 \\
6660.66 \\
6702.97 \\
6717.40 \\
6719.72\end{array}$ & $\begin{array}{l}\frac{1}{2}, \mathrm{~b} \\
1 \\
1 \\
1\end{array}$ & $\begin{array}{l}\text { B } \\
\text { B } \\
\text { B } \\
\text { B } \\
\text { B }\end{array}$ & $\begin{array}{l}6978.75 \\
6980.38 \\
6984.71 \\
6988.98 \\
6991.71\end{array}$ & $\begin{array}{l}3 \\
1 \\
1 \\
4 \\
2\end{array}$ & $\begin{array}{l}\mathbf{A} \\
\mathbf{A} \\
\mathbf{A} \\
\mathbf{A} \\
\mathbf{A}\end{array}$ & $\begin{array}{l}7329.00 \\
7331.51 \\
7333.69 \\
7333.92 \\
7339.61\end{array}$ & $\begin{array}{l}2, \mathrm{~h} \\
2, \mathrm{~h} \\
1 \\
1 \\
1\end{array}$ & $\begin{array}{l}\mathbf{B} \\
\mathbf{A} \\
\mathbf{A} \\
\mathbf{A} \\
\mathbf{E}\end{array}$ \\
\hline
\end{tabular}


TABLE 5-Continued

\begin{tabular}{|c|c|c|c|c|c|c|c|c|}
\hline$\lambda$ I. A. & Notes & $\begin{array}{c}\text { Probable } \\
\text { error }\end{array}$ & $\lambda$ I. A. & Notes & $\begin{array}{c}\text { Probable } \\
\text { error }\end{array}$ & $\lambda$ I. A. & Notes & $\begin{array}{l}\text { Probable } \\
\text { error }\end{array}$ \\
\hline $\begin{array}{l}7341.77 \\
7348.48 \\
7360.38 \\
7361.63 \\
7364.41\end{array}$ & $\begin{array}{l}1 \\
2 \\
1 \\
2 \\
2, d ?\end{array}$ & $\begin{array}{l}\text { B } \\
\text { B } \\
\text { B } \\
\text { B } \\
\text { C }\end{array}$ & $\begin{array}{l}7679.50 \\
7692.95 \\
7707.68 \\
7709.51 \\
7720.74\end{array}$ & $\begin{array}{l}2 \\
1, \mathrm{~h} \\
1 \\
2 \\
4\end{array}$ & $\begin{array}{l}\mathrm{B} \\
\mathrm{E} \\
\mathrm{B} \\
\mathrm{B} \\
\mathrm{B}\end{array}$ & $\begin{array}{l}8298.10 \\
8328.43 \\
8351.15 \\
8389.28 \\
8420.79\end{array}$ & $\begin{array}{l}2 \\
5 \\
2 \\
6 \\
1\end{array}$ & $\begin{array}{l}\mathbf{B} \\
\mathbf{B} \\
\mathbf{A} \\
\mathbf{B} \\
\mathbf{B}\end{array}$ \\
\hline $\begin{array}{l}7365.21 \\
7383.15 \\
7391.36 \\
7404.50 \\
7411.83\end{array}$ & $\begin{array}{l}2 \\
1 \\
5 \\
1 \\
1\end{array}$ & $\begin{array}{l}\mathbf{C} \\
\mathbf{B} \\
\mathbf{B} \\
\mathbf{B} \\
\mathbf{C}\end{array}$ & $\begin{array}{l}7723.62 \\
7730.56 \\
7732.47 \\
7745.86 \\
7748.93\end{array}$ & $\begin{array}{l}2 \\
1 \\
2 \\
1 \\
1\end{array}$ & $\begin{array}{l}\mathbf{B} \\
\mathbf{C} \\
\mathbf{B} \\
\mathbf{B} \\
\mathbf{B}\end{array}$ & $\begin{array}{l}8447.68 \\
8450.23 \\
8483.30 \\
8491.86 \\
8498.47\end{array}$ & $\begin{array}{l}1 \\
2, \mathrm{~h}, \mathrm{n} ? \\
2 \\
1 \\
1\end{array}$ & $\begin{array}{l}\mathbf{B} \\
\mathbf{B} \\
\mathbf{B} \\
\mathbf{B} \\
\mathbf{B}\end{array}$ \\
\hline $\begin{array}{l}7413.29 \\
7414.27 \\
7427.54 \\
7431.38 \\
7434.08\end{array}$ & $\begin{array}{l}1 \\
1 \\
1 \\
1 \\
2\end{array}$ & $\begin{array}{l}\mathbf{B} \\
\mathbf{B} \\
\mathbf{B} \\
\mathbf{B} \\
\mathbf{A}\end{array}$ & $\begin{array}{l}7752.29 \\
7753.88 \\
7774.53 \\
7776.03 \\
7784.46\end{array}$ & $\begin{array}{l}1 \\
1 \\
1 \\
1 \\
1\end{array}$ & $\begin{array}{l}\mathbf{B} \\
\mathbf{B} \\
\mathrm{E} \\
\mathbf{E} \\
\mathbf{E}\end{array}$ & $\begin{array}{l}8502.11 \\
8507.58 \\
8512.09 \\
8539.87 \\
8551.76\end{array}$ & $\begin{array}{l}1 \\
1 \\
1 \\
1 \\
1\end{array}$ & $\begin{array}{l}\mathrm{E} \\
\mathrm{B} \\
\mathrm{C} \\
\mathrm{E} \\
\mathrm{B}\end{array}$ \\
\hline $\begin{array}{l}7447.30 \\
7452.83 \\
7456.65 \\
7461.97 \\
7475.44\end{array}$ & $\begin{array}{l}2 \\
2 \\
1 \\
1 \\
1\end{array}$ & $\begin{array}{l}\text { B } \\
\text { A } \\
\text { A } \\
\text { B } \\
\text { A }\end{array}$ & $\begin{array}{l}7795.70 \\
7801.12 \\
7804.56 \\
7809.67 \\
7811.23\end{array}$ & $\begin{array}{l}1 \\
1 \\
1 \\
1 \\
1\end{array}$ & $\begin{array}{l}\mathbf{B} \\
\mathbf{E} \\
\mathbf{E} \\
\mathbf{E} \\
\mathbf{B}\end{array}$ & $\begin{array}{l}8553.71 \\
8556.72 \\
8594.56 \\
8599.36 \\
8601.15\end{array}$ & $\begin{array}{l}\frac{1}{1, b} \\
\frac{1}{2, v} \\
2, \mathrm{n} \\
1\end{array}$ & $\begin{array}{l}\mathbf{B} \\
\mathbf{E} \\
\mathbf{C} \\
\mathbf{B} \\
\mathbf{B}\end{array}$ \\
\hline $\begin{array}{l}7485.73 \\
7496.49 \\
7501.57 \\
7504.45 \\
7514.42\end{array}$ & $\begin{array}{l}7 \\
1 \\
1 \\
2 \\
1\end{array}$ & $\begin{array}{l}\mathbf{A} \\
\mathbf{E} \\
\mathbf{B} \\
\mathbf{B} \\
\mathbf{B}\end{array}$ & $\begin{array}{l}7829.63 \\
7854.44 \\
7861.96 \\
7867.22 \\
7869.36\end{array}$ & $\begin{array}{l}2 \\
2 \\
1 \\
1 \\
1\end{array}$ & $\begin{array}{l}\mathbf{A} \\
\mathbf{A} \\
\mathbf{E} \\
\mathbf{E} \\
\mathbf{B}\end{array}$ & $\begin{array}{l}8614.27 \\
8617.02 \\
8668.96 \\
8642.00 \\
8656.83\end{array}$ & $\begin{array}{l}2, n ? \\
\frac{1}{2} \\
1 \\
1, n ?\end{array}$ & $\begin{array}{l}\text { B } \\
\text { E } \\
\text { B } \\
\text { B } \\
C\end{array}$ \\
\hline $\begin{array}{l}7515.37 \\
7521.00 \\
7538.39 \\
7548.71 \\
7556.21\end{array}$ & $\begin{array}{l}1 \\
1 \\
1 \\
1 \\
1\end{array}$ & $\begin{array}{l}\text { B } \\
\text { A } \\
\text { B } \\
\text { C } \\
\text { B }\end{array}$ & $\begin{array}{l}7877.00 \\
7880.74 \\
7887.75 \\
7917.58 \\
7923.16\end{array}$ & $\begin{array}{l}1 \\
1 \\
2 \\
1 \\
2\end{array}$ & $\begin{array}{l}\mathbf{E} \\
\mathbf{E} \\
\mathbf{A} \\
\mathbf{B} \\
\mathbf{B}\end{array}$ & $\begin{array}{l}8694.56 \\
8695.53 \\
8714.18 \\
8731.72 \\
8763.60\end{array}$ & $\begin{array}{l}1 \\
2 \\
1 \\
1, h \\
1, h\end{array}$ & $\begin{array}{l}\mathbf{B} \\
\mathbf{B} \\
\mathbf{B} \\
\mathbf{E} \\
\mathbf{E}\end{array}$ \\
\hline $\begin{array}{l}7568.78 \\
7571.51 \\
7572.61 \\
7579.58 \\
7581.32\end{array}$ & $\begin{array}{l}1 \\
1 \\
2 \\
1 \\
1\end{array}$ & $\begin{array}{l}\mathbf{E} \\
\mathbf{B} \\
\mathbf{A} \\
\mathbf{A} \\
\mathbf{B}\end{array}$ & $\begin{array}{l}7949.02 \\
7968.84 \\
7984.32 \\
7986.58 \\
7992.56\end{array}$ & $\begin{array}{l}1 \\
1 \\
1 \\
2 \\
1\end{array}$ & $\begin{array}{l}\text { B } \\
\text { B } \\
\text { E } \\
\text { A } \\
\text { B }\end{array}$ & $\begin{array}{l}8765.70 \\
8777.35 \\
9067.46 \\
9123.83 \\
9177.21\end{array}$ & $\begin{array}{l}1 \\
1 \\
1 \\
1 \\
1\end{array}$ & $\begin{array}{l}\mathbf{E} \\
\mathbf{B} \\
\mathbf{B} \\
\mathbf{C} \\
\mathbf{C}\end{array}$ \\
\hline $\begin{array}{l}7583.61 \\
7591.67 \\
7595.16 \\
7601.82 \\
7617.39\end{array}$ & $\begin{array}{l}1 \\
1 \\
2 \\
2 \\
1\end{array}$ & $\begin{array}{l}\mathbf{A} \\
\mathbf{B} \\
\mathbf{A} \\
\mathbf{A} \\
\mathbf{A}\end{array}$ & $\begin{array}{l}8008.86 \\
8027.31 \\
8045.57 \\
8058.19 \\
8071.05\end{array}$ & $\begin{array}{l}1 \\
1 \\
1 \\
1 \\
1\end{array}$ & $\begin{array}{l}\mathbf{E} \\
\mathbf{B} \\
\mathbf{E} \\
\mathbf{B} \\
\mathbf{E}\end{array}$ & $\begin{array}{l}9252.91 \\
9348.01 \\
9422.72\end{array}$ & $\begin{array}{l}1 \\
2 \\
1 \\
1\end{array}$ & $\begin{array}{l}\text { C } \\
\text { B } \\
\text { D } \\
\text { C }\end{array}$ \\
\hline $\begin{array}{l}7618.78 \\
7621.72 \\
7624.98 \\
7631.46 \\
7647.13\end{array}$ & $\begin{array}{l}\text { 1, h } \\
1 \\
1 \\
1 \\
1\end{array}$ & $\begin{array}{l}\mathbf{E} \\
\mathbf{A} \\
\mathbf{B} \\
\mathbf{B} \\
\mathbf{C}\end{array}$ & $\begin{array}{l}8082.22 \\
8086.35 \\
8104.66 \\
8150.86 \\
8153.45\end{array}$ & $\begin{array}{l}1 \\
1 \\
1, g \\
1 \\
1\end{array}$ & $\begin{array}{l}\mathrm{E} \\
\mathrm{E} \\
\mathrm{C} \\
\mathrm{E} \\
\mathrm{E}\end{array}$ & $\begin{array}{l}9460.67 \\
9721.50\end{array}$ & & $\begin{array}{l}\mathrm{C} \\
\mathrm{E}\end{array}$ \\
\hline $\begin{array}{l}7649.52 \\
7563.26 \\
7656.74 \\
7664.24 \\
7676.63\end{array}$ & $\begin{array}{l}1 \\
1 \\
4 \\
1 \\
1\end{array}$ & $\begin{array}{l}\text { B } \\
\text { B } \\
\text { B } \\
\text { C } \\
\text { E }\end{array}$ & $\begin{array}{l}8192.54 \\
82203.76 \\
8212.06 \\
8245.06 \\
8257.34\end{array}$ & $\begin{array}{l}1 \\
1 \\
1 \\
3 \\
1\end{array}$ & $\begin{array}{l}\text { C } \\
\text { B } \\
\text { B } \\
\text { B } \\
\text { E }\end{array}$ & & & \\
\hline
\end{tabular}

\section{TUNGSTEN}

Between the limits 5500 A and 9200 A, 478 lines (Table 6) were measured in the arc spectrum of tungsterr. With few exceptions the lines are faint; that is, of intensity I or 2. Tungsten, like molybdenum, gives out a strong continuous spectrum, which adds to the difficulty of measurement. Impurity lines present were due solely to molybdenum, several of its stronger lines appearing faintly among the tungsten lines. 
The spectrum of tungsten to $6894 \mathrm{~A}$ has been photographed at Bonn by Fräulein Belke. ${ }^{18}$ Our measurements agree very closely with hers. A few pairs of lines with constant frequency differences of the type found by Paulson ${ }^{19}$ are listed below.

\begin{tabular}{|c|c|c|c|}
\hline$\lambda$ & & $\nu$ & $\Delta \nu$ \\
\hline 55856.0420 & 4 & 18228.09 & 94.97 \\
5514.77 & 7 & 18133.12 & \\
5978.91 & 2 & 16725.46 & 94.25 \\
6012.79 & 4 & 16631.21 & \\
6021.52 & 4 & 16607.10 & 94.28 \\
6055.90 & 1 & 16512.82 & \\
6820.25 & 4 & 14662.22 & 95.15 \\
6864.80 & 2 & 14567.07 & \\
7385.08 & 3 & 13540.81 & 94.91 \\
7437.21 & 1 & 13445.90 & \\
\hline
\end{tabular}

TABLE 6.-Arc Spectrum of Tungsten

\begin{tabular}{|c|c|c|c|c|c|c|c|c|}
\hline$\lambda$ I. A. & Notes & $\begin{array}{c}\text { Probable } \\
\text { error }\end{array}$ & $\lambda$ I. A. & Notes & $\begin{array}{c}\begin{array}{c}\text { Probable } \\
\text { error }\end{array} \\
\text { errots }\end{array}$ & $\lambda$ I. A. & Notes & $\begin{array}{c}\text { Probable } \\
\text { error }\end{array}$ \\
\hline $\begin{array}{l}5500.62 \\
5503.53 \\
5508.65 \\
5514.77 \\
5521.16\end{array}$ & $\begin{array}{l}2 \\
2 \\
1 \\
7 \\
1\end{array}$ & $\begin{array}{l}\mathrm{C} \\
\mathrm{C} \\
\mathrm{E} \\
\mathrm{C} \\
\mathrm{C}\end{array}$ & $\begin{array}{l}5747.26 \\
5749.22 \\
5750.26 \\
5753.41 \\
\mathbf{5 7 5 4 . 5 5}\end{array}$ & $\begin{array}{l}2 \\
2 \\
1 \\
1 \\
1\end{array}$ & $\begin{array}{l}\mathrm{B} \\
\mathrm{B} \\
\mathrm{B} \\
\mathrm{B} \\
\mathrm{B}\end{array}$ & $\begin{array}{l}5934.49 \\
5940.90 \\
5947.56 \\
5949.46 \\
5953.97\end{array}$ & $\begin{array}{l}1 \\
1 \\
4 \\
1 \\
1\end{array}$ & $\begin{array}{l}\text { B } \\
\text { B } \\
\text { B } \\
\text { E } \\
B\end{array}$ \\
\hline $\begin{array}{l}5531.54 \\
5533.26 \\
5537.80 \\
5539.60 \\
5568.12\end{array}$ & $\begin{array}{l}1 \\
1 \\
1 \\
1 \\
1\end{array}$ & $\begin{array}{l}\mathrm{C} \\
\mathrm{C} \\
\mathrm{C} \\
\mathrm{C} \\
\mathrm{B}\end{array}$ & $\begin{array}{l}5756.16 \\
5759.66 \\
5772.00 \\
5773.54 \\
5739.83\end{array}$ & $\begin{array}{ll}2 & \\
2 & \\
1 & \\
1 & \\
1\end{array}$ & $\begin{array}{l}\mathrm{B} \\
\mathrm{B} \\
\mathrm{B} \\
\mathrm{B} \\
\mathrm{C}\end{array}$ & $\begin{array}{l}5956.18 \\
5960.83 \\
5964.53 \\
5965.88 \\
5972.46\end{array}$ & $\begin{array}{l}2 \\
2 \\
1 \\
4 \\
2\end{array}$ & $\begin{array}{l}\mathrm{B} \\
\mathrm{B} \\
\mathrm{E} \\
\mathrm{B} \\
\mathrm{C}\end{array}$ \\
\hline $\begin{array}{l}5576.28 \\
5580.03 \\
5593.65 \\
5604.32 \\
5608.07\end{array}$ & $\begin{array}{l}1 \\
1 \\
1 \\
1 \\
1\end{array}$ & $\begin{array}{l}\mathrm{C} \\
\mathrm{E} \\
\mathrm{E} \\
\mathrm{D} \\
\mathrm{E}\end{array}$ & $\begin{array}{l}5791.36 \\
5793.02 \\
5796.54 \\
5799.53 \\
5803.10\end{array}$ & $\begin{array}{l}1 \\
2 \\
2 \\
1 \\
1\end{array}$ & $\begin{array}{l}\mathrm{B} \\
\mathrm{B} \\
\mathrm{C} \\
\mathrm{C} \\
\mathrm{E}\end{array}$ & $\begin{array}{l}5976.66 \\
5978.91 \\
5983.84 \\
5987.31 \\
5989.59\end{array}$ & $\begin{array}{l}2 \\
2 \\
2 \\
2, \mathrm{~h}, \mathrm{~d} ? \\
2, \mathrm{~d} ?\end{array}$ & $\begin{array}{l}\mathrm{B} \\
\mathrm{B} \\
\mathrm{B} \\
\mathrm{C} \\
\mathrm{C}\end{array}$ \\
\hline $\begin{array}{l}5616.21 \\
5617.33 \\
5629.75 \\
5631.30 \\
5631.94\end{array}$ & $\begin{array}{l}1 \\
2 \\
1 \\
1 \\
2\end{array}$ & $\begin{array}{l}\mathrm{E} \\
\mathrm{C} \\
\mathrm{C} \\
\mathrm{E} \\
\mathrm{B}\end{array}$ & $\begin{array}{l}5804.86 \\
5806.25 \\
5822.60 \\
5832.32 \\
5833.62\end{array}$ & $\begin{array}{l}4 \\
2 \\
2 \\
1 \\
2\end{array}$ & $\begin{array}{l}\text { C } \\
\text { C } \\
\text { B } \\
\text { B } \\
\text { B }\end{array}$ & $\begin{array}{l}5992.45 \\
5948.24 \\
6003.30 \\
6008.99 \\
6009.68\end{array}$ & $\begin{array}{l}1 \\
1 \\
1 \\
1 \\
1\end{array}$ & $\begin{array}{l}\mathrm{C} \\
\mathrm{E} \\
\mathrm{E} \\
\mathrm{B} \\
\mathrm{B}\end{array}$ \\
\hline $\begin{array}{l}5635.50 \\
5639.76 \\
5642.12 \\
5644.49 \\
5648.42\end{array}$ & $\begin{array}{l}1 \\
1 \\
2 \\
1 \\
4\end{array}$ & $\begin{array}{l}\mathrm{E} \\
\mathrm{E} \\
\mathrm{C} \\
\mathrm{E} \\
\mathrm{C}\end{array}$ & $\begin{array}{l}5838.99 \\
5845.28 \\
5851.59 \\
5854.45 \\
5855.64\end{array}$ & $\begin{array}{l}2 \\
2 \\
2 \\
1 \\
1 \\
1\end{array}$ & $\begin{array}{l}\mathrm{B} \\
\mathrm{C} \\
\mathrm{B} \\
\mathrm{C} \\
\mathrm{B}\end{array}$ & $\begin{array}{l}6012.79 \\
6021.52 \\
6028.32 \\
6029.83 \\
6035.52\end{array}$ & $\begin{array}{l}4 \\
4 \\
2 \\
1 \\
1\end{array}$ & $\begin{array}{l}\mathrm{A} \\
\mathrm{A} \\
\mathrm{B} \\
\mathrm{E} \\
\mathrm{E}\end{array}$ \\
\hline $\begin{array}{l}5660.67 \\
5664.40 \\
5673.41 \\
5674.45 \\
5675.38\end{array}$ & $\begin{array}{l}2 \\
2 \\
1 \\
2 \\
1\end{array}$ & $\begin{array}{l}\mathrm{C} \\
\mathrm{C} \\
\mathbf{C} \\
\mathbf{C} \\
\mathbf{C}\end{array}$ & $\begin{array}{l}5864.64 \\
5868.35 \\
5869.91 \\
5871.58 \\
5874.24\end{array}$ & $\begin{array}{l}2 \\
1, \mathbf{h} \\
1 \\
1 \\
1\end{array}$ & $\begin{array}{l}\mathrm{B} \\
\mathrm{E} \\
\mathrm{B} \\
\mathrm{C} \\
\mathrm{C}\end{array}$ & $\begin{array}{l}6037.33 \\
6041.62 \\
6043.33 \\
6046.66 \\
6049.90\end{array}$ & $\begin{array}{l}1 \\
2 \\
3 \\
1 \\
2\end{array}$ & $\begin{array}{l}B \\
B \\
B \\
C \\
C\end{array}$ \\
\hline $\begin{array}{l}5676.85 \\
5693.70 \\
5697.86 \\
5709.18 \\
5715.35\end{array}$ & $\begin{array}{l}2 \\
1 \\
2 \\
1 \\
1 \\
1\end{array}$ & $\begin{array}{l}\mathrm{C} \\
\mathrm{E} \\
\mathrm{C} \\
\mathrm{E} \\
\mathrm{E}\end{array}$ & $\begin{array}{l}5875.68 \\
5880.21 \\
5890.32 \\
5891.59 \\
5901.21\end{array}$ & $\begin{array}{l}1 \\
2 \\
1 \\
2 \\
1\end{array}$ & $\begin{array}{l}\mathrm{C} \\
\mathrm{B} \\
\mathrm{C} \\
\mathrm{B} \\
\mathrm{B}\end{array}$ & $\begin{array}{l}6053.18 \\
6055.90 \\
6057.45 \\
6065.05 \\
6066.82\end{array}$ & $\begin{array}{l}1 \\
1 \\
1 \\
2 \\
1\end{array}$ & $\begin{array}{l}\mathrm{E} \\
\mathrm{C} \\
\mathrm{E} \\
\mathrm{C} \\
\mathrm{E}\end{array}$ \\
\hline $\begin{array}{l}5723.19 \\
5728.60 \\
5735.11 \\
5739.59 \\
5741.20\end{array}$ & $\begin{array}{l}2 \\
1 \\
4 \\
2 \\
1\end{array}$ & $\begin{array}{l}\mathrm{B} \\
\mathrm{C} \\
\mathrm{B} \\
\mathrm{B} \\
\mathrm{C}\end{array}$ & $\begin{array}{l}5902.66 \\
5904.81 \\
5913.51 \\
5928.61 \\
5931.07\end{array}$ & $\begin{array}{l}2 \\
1, \mathrm{~d} ? \\
1 \\
2 \\
1\end{array}$ & $\begin{array}{l}\mathbf{B} \\
\mathbf{E} \\
\mathrm{E} \\
\mathrm{B} \\
\mathbf{E}\end{array}$ & $\begin{array}{l}6067.64 \\
6081.46 \\
6082.86 \\
6083.82 \\
6092.29\end{array}$ & $\begin{array}{l}1 \\
3 \\
1 \\
1 \\
1\end{array}$ & $\begin{array}{l}\mathrm{C} \\
\mathbf{C} \\
\mathbf{E} \\
\mathbf{C} \\
\mathrm{C}\end{array}$ \\
\hline
\end{tabular}

${ }^{13}$ Zeit. für Wiss. Phot., 17, pp. $\mathrm{r}_{32}$ and $\mathrm{I}_{45}$; $1918 .{ }^{20}$ Wave length given in Kayser's Handbuch

${ }^{19}$ Paulson, Dissertation, Lund; I9r4. 
TABLE 6-Continued

\begin{tabular}{|c|c|c|c|c|c|c|c|c|}
\hline$\lambda$ I. A. & Notes & $\begin{array}{c}\text { Probable } \\
\text { error }\end{array}$ & $\lambda$ I. A. & Notes & $\begin{array}{c}\text { Probable } \\
\text { error }\end{array}$ & $\lambda$ I. A. & Notes & $\begin{array}{l}\text { Probable } \\
\text { error }\end{array}$ \\
\hline $\begin{array}{l}6094.76 \\
6107.41 \\
6111.67 \\
6115.55 \\
6119.35\end{array}$ & $\begin{array}{l}1 \\
1 \\
2 \\
2 \\
1\end{array}$ & $\begin{array}{l}\text { C } \\
\text { B } \\
\text { B } \\
\text { A } \\
\text { A }\end{array}$ & $\begin{array}{l}6435.39 \\
6438.03 \\
6439.72 \\
6445.13 \\
6445.65\end{array}$ & $\begin{array}{l}2, \mathrm{~h}, \mathrm{~d} ? \\
1 \\
\frac{1}{2} \\
1\end{array}$ & $\begin{array}{l}\mathbf{B} \\
\mathbf{E} \\
\mathbf{B} \\
\mathbf{B} \\
\mathbf{E}\end{array}$ & $\begin{array}{l}6733.06 \\
6740.03 \\
6745.51 \\
6746.58 \\
6754.56\end{array}$ & $\begin{array}{l}2 \\
2 \\
1 \\
2 \\
1\end{array}$ & $\begin{array}{l}\text { A } \\
\text { B } \\
\text { B } \\
\text { A } \\
\text { B }\end{array}$ \\
\hline $\begin{array}{l}6128.28 \\
6129.60 \\
6133.17 \\
6140.97 \\
6143.91\end{array}$ & $\begin{array}{l}4 \\
1 \\
1 \\
1 \\
2\end{array}$ & $\begin{array}{l}\mathbf{A} \\
\mathbf{E} \\
\mathbf{A} \\
\mathbf{E} \\
\mathbf{B}\end{array}$ & $\begin{array}{l}6448.16 \\
6458.36 \\
6461.73 \\
6464.16 \\
6474.78\end{array}$ & $\begin{array}{l}3 \\
1 \\
1 \\
3 \\
1\end{array}$ & $\begin{array}{l}\text { C } \\
\mathbf{B} \\
\mathbf{E} \\
\mathbf{B} \\
\mathbf{A}\end{array}$ & $\begin{array}{l}6755.75 \\
6764.43 \\
6767.80 \\
6770.05 \\
6778.34\end{array}$ & $\begin{array}{l}1 \\
2 \\
1 \\
1 \\
2\end{array}$ & $\begin{array}{l}\mathbf{E} \\
\mathbf{A} \\
\mathbf{E} \\
\mathbf{E} \\
\mathbf{B}\end{array}$ \\
\hline $\begin{array}{l}6147.18 \\
6148.22 \\
6151.68 \\
6153.75 \\
6154.88\end{array}$ & $\begin{array}{l}1 \\
1 \\
2, \mathbf{h} \\
2 \\
2\end{array}$ & $\begin{array}{l}\mathbf{B} \\
\mathbf{B} \\
\mathbf{B} \\
\mathbf{B} \\
\mathbf{B}\end{array}$ & $\begin{array}{l}6479.54 \\
6484.09 \\
6491.53 \\
6494.85 \\
6500.29\end{array}$ & $\begin{array}{l}1 \\
2 \\
1 \\
1 \\
2, \mathbf{h}\end{array}$ & $\begin{array}{l}\text { B } \\
\text { B } \\
\text { B } \\
\text { C } \\
\text { B }\end{array}$ & $\begin{array}{l}6799.35 \\
6804.13 \\
6805.31 \\
6811.38 \\
6814.93\end{array}$ & $\begin{array}{l}2 \\
2 \\
3 \\
2 \\
3\end{array}$ & $\begin{array}{l}\text { B } \\
\mathbf{B} \\
\mathbf{A} \\
\mathbf{A} \\
\mathbf{A}\end{array}$ \\
\hline $\begin{array}{l}6161.45 \\
6169.11 \\
6173.78 \\
6179.66 \\
6186.22\end{array}$ & $\begin{array}{l}2 \\
2 \\
1 \\
1 \\
2\end{array}$ & $\begin{array}{l}\mathbf{B} \\
\mathrm{A} \\
\mathrm{E} \\
\mathrm{E} \\
\mathbf{B}\end{array}$ & $\begin{array}{l}6508.01 \\
6526.14 \\
6532.40 \\
6534.05 \\
6538.16\end{array}$ & $\begin{array}{l}2 \\
1 \\
3 \\
1 \\
4\end{array}$ & $\begin{array}{l}\text { A } \\
\text { B } \\
\text { A } \\
\text { B } \\
\text { A }\end{array}$ & $\begin{array}{l}6816.26 \\
6820.25 \\
6826.47 \\
6828.43 \\
6832.23\end{array}$ & $\begin{array}{l}2 \\
4 \\
1 \\
2 \\
1\end{array}$ & $\begin{array}{l}\mathbf{A} \\
\mathbf{A} \\
\mathbf{E} \\
\mathbf{B} \\
\mathbf{C}\end{array}$ \\
\hline $\begin{array}{l}6187.54 \\
6188.64 \\
6193.23 \\
6196.11 \\
6203.54\end{array}$ & $\begin{array}{l}1 \\
1 \\
1 \\
2, \mathrm{~h} \\
4\end{array}$ & $\begin{array}{l}\mathbf{A} \\
\mathrm{E} \\
\mathrm{B} \\
\mathrm{B} \\
\mathbf{B}\end{array}$ & $\begin{array}{l}6541.37 \\
6552.84 \\
6553.81 \\
6554.24 \\
6562.88\end{array}$ & $\begin{array}{l}1 \\
2 \\
1 \\
2 \\
1\end{array}$ & $\begin{array}{l}\mathbf{A} \\
\mathbf{A} \\
\mathbf{E} \\
\mathbf{A} \\
\mathbf{E}\end{array}$ & $\begin{array}{l}6838.61 \\
6840.07 \\
6844.88 \\
6847.21 \\
6853.73\end{array}$ & $\begin{array}{l}1 \\
1 \\
1 \\
2 \\
2\end{array}$ & $\begin{array}{l}\mathbf{B} \\
\mathbf{E} \\
\mathbf{B} \\
\mathbf{A} \\
\mathbf{A}\end{array}$ \\
\hline $\begin{array}{l}6216.51 \\
6219.50 \\
6222.67 \\
6224.21 \\
6225.80\end{array}$ & $\begin{array}{l}1 \\
1, \mathrm{~h} \\
1 \\
1 \\
1\end{array}$ & $\begin{array}{l}\mathbf{E} \\
\mathbf{E} \\
\mathbf{E} \\
\mathbf{E} \\
\mathbf{B}\end{array}$ & $\begin{array}{l}6563.16 \\
6567.71 \\
6569.75 \\
6571.82 \\
6573.96\end{array}$ & $\begin{array}{l}3 \\
2 \\
1, h \\
1, h \\
3\end{array}$ & $\begin{array}{l}\mathbf{A} \\
\mathbf{A} \\
\mathbf{A} \\
\mathbf{A} \\
\mathbf{A}\end{array}$ & $\begin{array}{l}6864.80 \\
6874.93 \\
6876.05 \\
6877.60 \\
6891.53\end{array}$ & $\begin{array}{l}2 \\
2 \\
2 \\
2 \\
1, \mathrm{~h}, \mathrm{~d} ?\end{array}$ & $\begin{array}{l}\mathbf{A} \\
\mathbf{A} \\
\mathbf{A} \\
\mathbf{A} \\
\mathbf{B}\end{array}$ \\
\hline $\begin{array}{l}6237.78 \\
6245.74 \\
6251.49 \\
6253.62 \\
6254.33\end{array}$ & $\begin{array}{l}1 \\
1 \\
1 \\
1 \\
3\end{array}$ & $\begin{array}{l}\mathbf{E} \\
\mathbf{B} \\
\mathbf{B} \\
\mathbf{E} \\
\mathbf{B}\end{array}$ & $\begin{array}{l}6582.90 \\
6600.06 \\
6607.17 \\
6608.49 \\
6609.06\end{array}$ & $\begin{array}{ll}2 & . \\
2 & \\
2 & \\
2 & \\
2 & \end{array}$ & $\begin{array}{l}\mathbf{A} \\
\mathbf{A} \\
\mathbf{A} \\
\mathbf{B} \\
\mathbf{B}\end{array}$ & $\begin{array}{l}6900.47 \\
6908.31 \\
6915.49 \\
6917.21 \\
6920.98\end{array}$ & $\begin{array}{l}1 \\
3 \\
1 \\
1 \\
2\end{array}$ & $\begin{array}{l}\mathbf{A} \\
\mathbf{A} \\
\mathbf{A} \\
\mathbf{A} \\
\mathbf{A}\end{array}$ \\
\hline $\begin{array}{l}6258.93 \\
6269.19 \\
6279.51 \\
6285.89 \\
6288.90\end{array}$ & $\begin{array}{l}2, \mathrm{~h} \\
1 \\
1 \\
4 \\
1, \mathrm{~h}\end{array}$ & $\begin{array}{l}\mathbf{B} \\
\mathbf{C} \\
\mathrm{B} \\
\mathrm{B} \\
\mathrm{E}\end{array}$ & $\begin{array}{l}6611.63 \\
6620.60 \\
6621.69 \\
6622.39 \\
6636.60\end{array}$ & $\begin{array}{l}3 \\
1 \\
3 \\
1 \\
1, \mathbf{h}\end{array}$ & $\begin{array}{l}\mathrm{A} \\
\mathrm{B} \\
\mathrm{B} \\
\mathrm{B} \\
\mathrm{E} \\
\mathrm{B}\end{array}$ & $\begin{array}{l}6934.29 \\
6943.72 \\
6955.57 \\
6964.14 \\
6971.67\end{array}$ & $\begin{array}{l}4 \\
1 \\
2 \\
3 \\
2\end{array}$ & $\begin{array}{l}\mathbf{A} \\
\mathbf{B} \\
\mathbf{A} \\
\mathbf{A} \\
\mathbf{A}\end{array}$ \\
\hline $\begin{array}{l}6291.04 \\
6292.02 \\
6297.14 \\
6298.57 \\
6303.18\end{array}$ & $\begin{array}{l}3 \\
4 \\
1 \\
1 \\
2\end{array}$ & $\begin{array}{l}\text { C } \\
\text { B } \\
\text { B } \\
\text { B } \\
\text { B }\end{array}$ & $\begin{array}{l}6639.15 \\
6643.54 \\
6643.84 \\
6651.78 \\
6653.96\end{array}$ & $\begin{array}{l}1, \mathrm{~h} \\
1, \mathrm{~h} \\
1, \mathrm{~h} \\
1\end{array}$ & $\begin{array}{l}\mathbf{E} \\
\mathbf{E} \\
\mathbf{E} \\
\mathbf{E} \\
\mathbf{E}\end{array}$ & $\begin{array}{l}6984.28 \\
6989.31 \\
6991.54 \\
6993.15 \\
6994.06\end{array}$ & $\begin{array}{l}4 \\
1 \\
1 \\
2, \mathrm{~d} \\
2\end{array}$ & $\begin{array}{l}\mathbf{A} \\
\mathbf{A} \\
\mathbf{A} \\
\mathbf{A} \\
\mathbf{A}\end{array}$ \\
\hline $\begin{array}{l}6308.47 \\
6319.23 \\
6331.38 \\
6342.31 \\
6355.84\end{array}$ & $\begin{array}{l}1 \\
1 \\
1 \\
1 \\
2\end{array}$ & $\begin{array}{l}\mathbf{B} \\
\mathbf{B} \\
\mathbf{B} \\
\mathbf{B} \\
\mathbf{B}\end{array}$ & $\begin{array}{l}6654.45 \\
6656.51 \\
6657.02 \\
6659.14 \\
6664.16\end{array}$ & $\begin{array}{l}1, \mathbf{h} \\
1, \mathbf{h} \\
1 \\
1 \\
1, \mathbf{h}\end{array}$ & $\begin{array}{l}\mathbf{E} \\
\mathbf{E} \\
\mathbf{E} \\
\mathbf{B} \\
\mathbf{E}\end{array}$ & $\begin{array}{l}7002.73 \\
7009.72 \\
7016.01 \\
7017.92 \\
7020.23\end{array}$ & $\begin{array}{l}1 \\
1 \\
1, \mathrm{~d} \\
2 \\
1, \mathrm{~h}\end{array}$ & $\begin{array}{l}\mathbf{A} \\
\mathbf{B} \\
\mathbf{A} \\
\mathbf{A} \\
\mathbf{B}\end{array}$ \\
\hline $\begin{array}{l}6357.78 \\
6359.15 \\
6361.07 \\
6364.77 \\
6370.28\end{array}$ & $\begin{array}{l}1 \\
1 \\
1 \\
1 \\
1\end{array}$ & $\begin{array}{l}\text { E } \\
\text { B } \\
\text { B } \\
\text { B } \\
\text { B }\end{array}$ & $\begin{array}{l}6664.83 \\
6675.99 \\
6677.19 \\
6678.41 \\
6682.65\end{array}$ & $\begin{array}{l}2 \\
1 \\
2 \\
4 \\
1\end{array}$ & $\begin{array}{l}\mathbf{B} \\
\mathbf{B} \\
\mathbf{B} \\
\mathbf{B} \\
\mathbf{B}\end{array}$ & $\begin{array}{l}7028.67 \\
7044.18 \\
7056.28 \\
7058.79 \\
7061.29\end{array}$ & $\begin{array}{l}1 \\
1, \mathrm{~h} \\
1, \mathrm{~h} \\
1, \mathrm{~h} \\
2, \mathrm{~h}\end{array}$ & $\begin{array}{l}\mathbf{A} \\
\mathbf{E} \\
\mathbf{B} \\
\mathbf{B} \\
\mathbf{B}\end{array}$ \\
\hline $\begin{array}{l}6371.43 \\
6375.37 \\
6382.51 \\
6384.68 \\
6386.47\end{array}$ & $\begin{array}{l}2 \\
2 \\
1 \\
2 \\
2\end{array}$ & $\begin{array}{l}\mathbf{A} \\
\mathbf{B} \\
\mathbf{B} \\
\mathbf{B} \\
\mathbf{B}\end{array}$ & $\begin{array}{l}6693.11 \\
6701.79 \\
6705.68 \\
6708.18 \\
6709.24\end{array}$ & $\begin{array}{l}5 \\
1 \\
1 \\
1 \\
1\end{array}$ & $\begin{array}{l}\mathbf{B} \\
\mathrm{E} \\
\mathbf{E} \\
\mathrm{E} \\
\mathbf{E}\end{array}$ & $\begin{array}{l}7071.05 \\
7089.32 \\
7098.23 \\
7111.20 \\
7116.90\end{array}$ & $\begin{array}{l}1, \mathbf{h} \\
1 \\
2 \\
1 \\
1\end{array}$ & $\begin{array}{l}\text { C } \\
\text { B } \\
\text { B } \\
\text { B } \\
\text { E }\end{array}$ \\
\hline $\begin{array}{l}6399.76 \\
640.27 \\
6404.22 \\
6416.02 \\
6425.36\end{array}$ & $\begin{array}{l}1 \\
2 \\
4 \\
2 \\
1\end{array}$ & $\begin{array}{l}\text { B } \\
\text { A } \\
\text { B } \\
\text { B } \\
\text { B }\end{array}$ & $\begin{array}{l}6710.58 \\
6713.30 \\
6718.57 \\
6719.88 \\
6725.19\end{array}$ & $\begin{array}{l}1 \\
2 \\
1 \\
1 \\
2\end{array}$ & $\begin{array}{l}\text { E } \\
\text { B } \\
\text { B } \\
\text { B } \\
\text { A }\end{array}$ & $\begin{array}{l}7127.07 \\
7140.51 \\
7148.94 \\
7162.64 \\
7166.93\end{array}$ & $\begin{array}{l}1 \\
3 \\
1 \\
2 \\
1\end{array}$ & $\begin{array}{l}\mathbf{B} \\
\mathbf{A} \\
\mathbf{E} \\
\mathbf{A} \\
\mathbf{B}\end{array}$ \\
\hline
\end{tabular}


TABLE 6-Continued

\begin{tabular}{|c|c|c|c|c|c|c|c|c|}
\hline$\lambda$ 入 I. A. & Notes & $\begin{array}{c}\text { Probable } \\
\text { error }\end{array}$ & $\lambda$ I. A. & Notes & $\begin{array}{c}\text { Probable } \\
\text { error }\end{array}$ & $\lambda$ I. A. & Notes & $\begin{array}{c}\text { Probable } \\
\text { error }\end{array}$ \\
\hline $\begin{array}{l}7179.88 \\
7184.71 \\
7187.57 \\
7191.33 \\
7198.62\end{array}$ & $\begin{array}{l}1 \\
1, \mathrm{~h} \\
1 \\
1 \\
1\end{array}$ & $\begin{array}{c}\mathbf{E} \\
\mathbf{E} \\
\mathbf{E} \\
\mathbf{A} \\
\mathbf{A}\end{array}$ & $\begin{array}{l}7630.24 \\
7631.29 \\
7643.40 \\
7653.62 \\
7654.78\end{array}$ & $\begin{array}{l}1 \\
1 \\
1 \\
1 \\
2\end{array}$ & $\begin{array}{l}\mathbf{B} \\
\mathbf{A} \\
\mathbf{B} \\
\mathbf{B} \\
\mathbf{B}\end{array}$ & $\begin{array}{l}8139.57 \\
8143.14 \\
8165.71 \\
8210.20 \\
8222.52\end{array}$ & $\begin{array}{l}1, \mathrm{~h} \\
2 \\
1 \\
2, \mathrm{Ba} ? \\
1, \mathrm{~h}\end{array}$ & $\begin{array}{l}\mathbf{A} \\
\mathbf{A} \\
\mathbf{A} \\
\mathbf{A} \\
\mathbf{A}\end{array}$ \\
\hline $\begin{array}{l}7200.16 \\
7216.32 \\
7218.16 \\
7220.14 \\
7226.02\end{array}$ & $\begin{array}{l}2 \\
2 \\
1 \\
1 \\
2\end{array}$ & $\begin{array}{l}\mathbf{A} \\
\mathbf{A} \\
\mathbf{C} \\
\mathbf{B} \\
\mathbf{B}\end{array}$ & $\begin{array}{l}7664.86 \\
7672.32 \\
7675.59 \\
7679.15 \\
7688.93\end{array}$ & $\begin{array}{l}3, \mathrm{~K} ? \\
1 \\
1 \\
1 \\
3\end{array}$ & $\begin{array}{l}\mathbf{B} \\
\mathbf{B} \\
\mathbf{E} \\
\mathbf{B} \\
\mathbf{B}\end{array}$ & $\begin{array}{l}8311.60 \\
8317.18 \\
8321.98 \\
8338.02 \\
8348.77\end{array}$ & $\begin{array}{l}1 \\
1 \\
1 \\
2 \\
1\end{array}$ & $\begin{array}{l}\mathbf{B} \\
\mathbf{E} \\
\mathbf{A} \\
\mathbf{A} \\
\mathbf{A}\end{array}$ \\
\hline $\begin{array}{l}7230.35 \\
7231.68 \\
7237.08 \\
7245.23 \\
7274.47\end{array}$ & $\begin{array}{l}1 \\
1 \\
2 \\
1 \\
2, \nabla\end{array}$ & $\begin{array}{l}\mathbf{E} \\
\mathbf{E} \\
\mathbf{A} \\
\mathbf{B} \\
\mathbf{B}\end{array}$ & $\begin{array}{l}7689.96 \\
7698.00 \\
7699.42 \\
7700.96 \\
7736.16\end{array}$ & $\begin{array}{l}1 \\
1 \\
1 \\
2 \\
1\end{array}$ & $\begin{array}{l}\text { B } \\
\text { B } \\
\text { B } \\
\text { B } \\
\text { E }\end{array}$ & $\begin{array}{l}8350.25 \\
8358.66 \\
8359.81 \\
8368.23 \\
8368.86\end{array}$ & $\begin{array}{l}1 \\
2 \\
1 \\
1 \\
1\end{array}$ & $\begin{array}{l}\mathbf{E} \\
\mathbf{A} \\
\mathbf{E} \\
\mathrm{E} \\
\mathbf{E}\end{array}$ \\
\hline $\begin{array}{l}7278.21 \\
7285.82 \\
7291.83 \\
7295.15 \\
7296.57\end{array}$ & $\begin{array}{l}2 \\
3 \\
1, \mathrm{~h} \\
1 \\
3\end{array}$ & $\begin{array}{l}\mathbf{A} \\
\mathbf{A} \\
\mathbf{B} \\
\mathbf{B} \\
\mathbf{A}\end{array}$ & $\begin{array}{l}7746.06 \\
7754.90 \\
7758.58 \\
7761.13 \\
7776.67\end{array}$ & $\begin{array}{l}1 \\
1 \\
1 \\
2 \\
2\end{array}$ & $\begin{array}{l}\text { B } \\
\text { E } \\
\text { B } \\
\text { B } \\
\text { B }\end{array}$ & $\begin{array}{l}8382.88 \\
8402.53 \\
8409.50 \\
8417.08 \\
8431.91\end{array}$ & $\begin{array}{l}2 \\
2 \\
1 \\
2 \\
2\end{array}$ & $\begin{array}{l}\mathrm{A} \\
\mathrm{A} \\
\mathrm{B} \\
\mathrm{E} \\
\mathrm{B} \\
\mathrm{C}\end{array}$ \\
\hline $\begin{array}{l}7298.23 \\
7347.88 \\
7355.00 \\
7361.70 \\
7365.15\end{array}$ & $\begin{array}{l}1 \\
1 \\
1 \\
1 \\
1\end{array}$ & $\begin{array}{l}\mathbf{B} \\
\mathbf{B} \\
\mathbf{B} \\
\mathbf{B} \\
\mathbf{B}\end{array}$ & $\begin{array}{l}7784.11 \\
7808.95 \\
7823.76 \\
7827.36 \\
7839.45\end{array}$ & $\begin{array}{l}3 \\
2 \\
1 \\
1 \\
1, h\end{array}$ & $\begin{array}{l}\mathbf{B} \\
\mathbf{A} \\
\mathbf{B} \\
\mathbf{C} \\
\mathbf{C}\end{array}$ & $\begin{array}{l}8470.92 \\
8475.15 \\
8486.81 \\
8495.06 \\
8495.68\end{array}$ & $\begin{array}{l}2 \\
3 \\
1 \\
2, \mathrm{~h} \\
1\end{array}$ & $\begin{array}{l}\mathbf{B} \\
\mathbf{B} \\
\mathbf{B} \\
\mathbf{B} \\
\mathbf{E}\end{array}$ \\
\hline $\begin{array}{l}7368.39 \\
7381.27 \\
7384.04 \\
7385.08 \\
7389.00\end{array}$ & $\begin{array}{l}1 \\
2 \\
1 \\
3 \\
1\end{array}$ & $\begin{array}{l}\mathbf{B} \\
\mathbf{B} \\
\mathbf{B} \\
\mathbf{B} \\
\mathbf{B}\end{array}$ & $\begin{array}{l}7863.45 \\
7867.01 \\
7877.61 \\
7878.56 \\
7880.34\end{array}$ & $\begin{array}{l}2 \\
2 \\
1 \\
1 \\
2\end{array}$ & $\begin{array}{l}\mathbf{A} \\
\mathbf{A} \\
\mathbf{E} \\
\mathbf{E} \\
\mathbf{A}\end{array}$ & $\begin{array}{l}8497.37 \\
8499.60 \\
8506.95 \\
8515.36 \\
8516.37\end{array}$ & $\begin{array}{l}1 \\
1 \\
2 \\
2 \\
2\end{array}$ & $\begin{array}{l}\mathbf{E} \\
\mathbf{E} \\
\mathbf{B} \\
\mathbf{B} \\
\mathbf{B}\end{array}$ \\
\hline $\begin{array}{l}7410.82 \\
7429.03 \\
7431.36 \\
7433.47 \\
7437.21\end{array}$ & $\begin{array}{l}1 \\
1 \\
1 \\
1 \\
1\end{array}$ & $\begin{array}{l}\mathbf{E} \\
\mathbf{C} \\
\mathbf{E} \\
\mathbf{E} \\
\mathbf{B}\end{array}$ & $\begin{array}{l}7883.17 \\
7886.45 \\
7905.25 \\
7909.19 \\
7914.28\end{array}$ & $\begin{array}{l}1 \\
2 \\
2 \\
2 \\
1, \mathrm{~h}\end{array}$ & $\begin{array}{l}\mathbf{C} \\
\mathbf{A} \\
\mathbf{B} \\
\mathbf{B} \\
\mathbf{B}\end{array}$ & $\begin{array}{l}8530.99 \\
8577.75 \\
8585.07 \\
8594.38 \\
8613.22\end{array}$ & $\begin{array}{l}2 \\
1 \\
3 \\
3 \\
3\end{array}$ & $\begin{array}{l}\mathbf{B} \\
\mathbf{E} \\
\mathbf{A} \\
\mathbf{A} \\
\mathbf{B}\end{array}$ \\
\hline $\begin{array}{l}7439.45 \\
7448.26 \\
7451.37 \\
7456.36 \\
7459.23\end{array}$ & $\begin{array}{l}1 \\
1 \\
2 \\
1 \\
1\end{array}$ & $\begin{array}{l}\text { B } \\
\text { B } \\
\text { B } \\
\text { B } \\
\text { B }\end{array}$ & $\begin{array}{l}7940.92 \\
7955.43 \\
7957.05 \\
7967.16 \\
7975.40\end{array}$ & $\begin{array}{l}3 \\
1 \\
2 \\
1 \\
1\end{array}$ & $\begin{array}{l}\mathbf{A} \\
\mathbf{B} \\
\mathbf{B} \\
\mathbf{E} \\
\mathbf{E}\end{array}$ & $\begin{array}{l}8614.49 \\
8641.56 \\
8710.76 \\
8740.44 \\
8744.64\end{array}$ & $\begin{array}{l}2 \\
2 \\
1 \\
2 \\
2\end{array}$ & $\begin{array}{l}\mathbf{B} \\
\mathbf{C} \\
\mathbf{C} \\
\mathbf{B} \\
\mathbf{E}\end{array}$ \\
\hline $\begin{array}{l}7468.16 \\
7471.55 \\
7483.34 \\
7504.07 \\
7508.98\end{array}$ & $\begin{array}{l}1 \\
1 \\
3 \\
2 \\
3\end{array}$ & $\begin{array}{l}\mathbf{B} \\
\mathbf{B} \\
\mathbf{B} \\
\mathbf{A} \\
\mathbf{A}\end{array}$ & $\begin{array}{l}7985.59 \\
7991.67 \\
8017.17 \\
8022.38 \\
8039.04\end{array}$ & $\begin{array}{l}1 \\
1 \\
4 \\
1 \\
1\end{array}$ & $\begin{array}{l}\mathbf{E} \\
\mathbf{E} \\
\mathbf{B} \\
\mathbf{E} \\
\mathbf{E}\end{array}$ & $\begin{array}{l}8746.59 \\
8754.83 \\
8776.45 \\
8777.45\end{array}$ & $\begin{array}{l}2 \\
1 \\
1 \\
1\end{array}$ & $\begin{array}{l}\mathbf{B} \\
\mathbf{E} \\
\mathbf{E} \\
\mathbf{E}\end{array}$ \\
\hline $\begin{array}{l}7520.63 \\
7521.59 \\
7525.44 \\
7537.42 \\
7550.46\end{array}$ & $\begin{array}{l}1 \\
1 \\
1 \\
3 \\
2\end{array}$ & $\begin{array}{l}\mathbf{A} \\
\mathbf{E} \\
\mathbf{E} \\
\mathbf{B} \\
\mathbf{A}\end{array}$ & $\begin{array}{l}8043.11 \\
8054.87 \\
8055.61 \\
8058.14 \\
8060.35\end{array}$ & $\begin{array}{l}1 \\
1 \\
3 \\
1 \\
2\end{array}$ & $\begin{array}{l}\mathbf{E} \\
\mathbf{B} \\
\mathbf{B} \\
\mathbf{E} \\
\mathbf{B}\end{array}$ & $\begin{array}{l}8823.39 \\
8865.50 \\
8871.65 \\
8883.66\end{array}$ & $\begin{array}{l}1 \\
1 \\
2 \\
1 \\
1\end{array}$ & $\begin{array}{l}\mathrm{d} \\
\mathrm{C} \\
\mathrm{C}\end{array}$ \\
\hline $\begin{array}{l}7569.87 \\
7582.85 \\
7604.74 \\
7612.13 \\
7614.07\end{array}$ & $\begin{array}{l}3 \\
2 \\
1, h \\
2 \\
3\end{array}$ & $\begin{array}{l}\mathbf{A} \\
\mathbf{A} \\
\mathbf{E} \\
\mathbf{A} \\
\mathbf{A}\end{array}$ & $\begin{array}{l}8063.33 \\
8072.12 \\
8091.08 \\
8123.78 \\
8132.37\end{array}$ & $\begin{array}{l}1, \mathrm{~h} \\
1, \mathrm{~h}, \mathrm{n} ? \\
\frac{1}{3} \\
1, \mathrm{~h}\end{array}$ & $\begin{array}{l}\mathbf{E} \\
\mathbf{B} \\
\mathbf{A} \\
\mathbf{A} \\
\mathbf{A}\end{array}$ & $\begin{array}{l}8984.21 \\
9158.87\end{array}$ & $\begin{array}{l}1, \mathbf{h} \\
1, \mathbf{h}\end{array}$ & $\underset{\mathbf{C}}{\breve{E}}$ \\
\hline
\end{tabular}

\section{URANIUM}

The spectrum of uranium is very rich in lines, but none of them are very strong. Like tungsten and molybdenum, uranium emits a continuous spectrum, which renders the spectrograms difficult of measurement. Meissner ${ }^{21}$ has published some wave lengths in 
the infra-red portion of the spectrum with which ours agree. Eder and Valenta ${ }^{22}$ have measured the spectrum to 7 I $30 \mathrm{~A}$ on Rowland's scale. A number of lines given by these observers and designated as "verbreitert," were not measured by us, perhaps because they were indistinguishable from the background of continuous spectrum.

A brief survey of qur measurements has shown a number of pairs and groups of lines with constant frequency differences. They are:

\begin{tabular}{|c|c|c|c|c|c|c|c|}
\hline$\lambda$ & & $\nu$ & $\Delta \nu$ & $\lambda$ & & $\nu$ & $\Delta \nu$ \\
\hline $\begin{array}{l}5610.90 \\
5620.73\end{array}$ & $\begin{array}{l}2 \\
2\end{array}$ & $\begin{array}{l}17822.45 \\
17791.29\end{array}$ & 31.16 & $\begin{array}{l}7761.82 \\
7780.49\end{array}$ & $\begin{array}{l}2 \\
1\end{array}$ & $\begin{array}{l}12883.58 \\
12852.66\end{array}$ & 30.92 \\
\hline $\begin{array}{l}5634.37 \\
5644.28\end{array}$ & $\begin{array}{l}2 \\
1\end{array}$ & $\begin{array}{l}17748.21 \\
17717.05\end{array}$ & 31.16 & $\begin{array}{l}6378.46 \\
6379.61\end{array}$ & $\begin{array}{l}2 \\
2\end{array}$ & $\begin{array}{l}15677.76 \\
15674.94\end{array}$ & 2.82 \\
\hline $\begin{array}{l}5705.60 \\
5715.71\end{array}$ & $\begin{array}{l}1 \\
1\end{array}$ & $\begin{array}{l}17526.64 \\
17 \quad 495.64\end{array}$ & 31.00 & $\begin{array}{l}6620.50 \\
6621.73\end{array}$ & $\begin{array}{l}2 \\
2\end{array}$ & $\begin{array}{ll}15 & 104.60 \\
15 & 101.79\end{array}$ & 2.81 \\
\hline $\begin{array}{l}5800.80 \\
5811.28\end{array}$ & $\begin{array}{l}1 \\
1\end{array}$ & $\begin{array}{l}17239.00 \\
17207.91\end{array}$ & 31.09 & $\begin{array}{l}7970.44 \\
7975.15 \\
7976.86\end{array}$ & $\begin{array}{l}3 \\
2 \\
1\end{array}$ & $\begin{array}{ll}12 & 546.36 \\
12 & 538.95 \\
12 & 536.26\end{array}$ & $\begin{array}{l}7.41 \\
2.69\end{array}$ \\
\hline $\begin{array}{l}5843.29 \\
5853.93\end{array}$ & $\begin{array}{l}1 \\
1\end{array}$ & $\begin{array}{l}17113.65 \\
17082.54\end{array}$ & 31.11 & $\begin{array}{l}8381.92 \\
8387.25\end{array}$ & 2 & $\begin{array}{l}11930.44 \\
11922.86\end{array}$ & 7.58 \\
\hline $\begin{array}{l}5986.15 \\
5997.32\end{array}$ & $\begin{array}{l}2 \\
2\end{array}$ & $\begin{array}{l}16705.23 \\
16674.11\end{array}$ & 31.12 & 8389.12 & 2 & 11920.20 & \\
\hline $\begin{array}{l}6378.46 \\
6391.11\end{array}$ & $\begin{array}{l}2 \\
1, \mathrm{~h}\end{array}$ & $\begin{array}{l}15677.76 \\
15646.73\end{array}$ & 31.03 & & & . & \\
\hline
\end{tabular}

TABLE 7.-Arc Spectrum of Uranium

\begin{tabular}{|c|c|c|c|c|c|c|c|c|}
\hline$\lambda$ I. A. & Notes & $\begin{array}{c}\text { Probable } \\
\text { error }\end{array}$ & $\lambda$ I. A. & Notes & $\begin{array}{l}\text { Probable } \\
\text { error }\end{array}$ & $\lambda$ I. A. & Notes & $\begin{array}{c}\text { Probable } \\
\text { error }\end{array}$ \\
\hline $\begin{array}{l}5504.16 \\
5510.44 \\
5511.54 \\
5513.40 \\
5527.86\end{array}$ & $\begin{array}{l}1 \\
1 \\
2 \\
1 \\
3\end{array}$ & $\begin{array}{l}\text { C } \\
\text { B } \\
\text { C } \\
\text { B } \\
B\end{array}$ & $\begin{array}{l}5614.86 \\
5616.68 \\
5620.73 \\
5621.53 \\
5624.52\end{array}$ & $\begin{array}{l}\frac{1}{1} \\
1, \mathrm{~h} \\
2 \\
1 \\
1\end{array}$ & $\begin{array}{l}\mathrm{E} \\
\mathrm{B} \\
\mathrm{B} \\
\mathrm{B} \\
\mathrm{E}\end{array}$ & $\begin{array}{l}5704.11 \\
5705.60 \\
5706.95 \\
5709.56 \\
5714.28\end{array}$ & $\begin{array}{l}1 \\
1 \\
1, \mathrm{~b} \\
1 \\
1\end{array}$ & $\begin{array}{l}\text { C } \\
\mathbf{C} \\
\mathbf{B} \\
\mathbf{E} \\
\mathbf{E}\end{array}$ \\
\hline $\begin{array}{l}5531.22 \\
5538.59 \\
5544.76 \\
5548.04 \\
5551.41\end{array}$ & $\begin{array}{l}1, v \\
1 \\
1 \\
1 \\
1\end{array}$ & $\begin{array}{l}\text { C } \\
\text { B } \\
\text { B } \\
\text { B } \\
\text { B }\end{array}$ & $\begin{array}{l}5628.01 \\
5629.58 \\
5634.37 \\
5636.78 \\
5637.95\end{array}$ & $\begin{array}{l}1, \mathrm{~b}, \mathrm{v} \\
1, \mathrm{v} \\
2 \\
1 \\
1\end{array}$ & $\begin{array}{l}\mathrm{B} \\
\mathbf{C} \\
\mathbf{C} \\
\mathbf{B} \\
\mathbf{B}\end{array}$ & $\begin{array}{l}5715.20 \\
5715.71 \\
5716.89 \\
5718.99 \\
5721.70\end{array}$ & $\begin{array}{l}1, \mathrm{~g} ? \\
1 \\
1 \\
1 \\
1\end{array}$ & $\begin{array}{l}\mathbf{B} \\
\mathbf{B} \\
\mathbf{B} \\
\mathbf{E} \\
\mathbf{E}\end{array}$ \\
\hline $\begin{array}{l}5552.66 \\
5555.90 \\
5557.91 \\
5564.14 \\
5570.72\end{array}$ & $\begin{array}{l}1 \\
1 \\
1 \\
3 \\
1\end{array}$ & $\begin{array}{l}\text { C } \\
\text { E } \\
\text { B } \\
\text { B } \\
\text { C }\end{array}$ & $\begin{array}{l}5640.38 \\
5644.28 \\
5648.45 \\
5653.73 \\
5654.49\end{array}$ & $\begin{array}{l}1 \\
1 \\
1 \\
1 \\
1\end{array}$ & $\begin{array}{l}B \\
E \\
E \\
C \\
C\end{array}$ & $\begin{array}{l}5722.31 \\
5723.64 \\
5733.34 \\
5736.36 \\
5737.27\end{array}$ & $\begin{array}{l}1 \\
2 \\
1 \\
1 \\
1\end{array}$ & $\begin{array}{l}\mathbf{C} \\
\mathbf{C} \\
\mathbf{C} \\
\mathbf{B} \\
\mathbf{B}\end{array}$ \\
\hline $\begin{array}{l}5580.92 \\
5581.41 \\
5581.69 \\
5587.18 \\
5597.35\end{array}$ & $\begin{array}{l}1 \\
2, v \\
2 \\
1 \\
1\end{array}$ & $\begin{array}{l}\mathrm{C} \\
\mathrm{D} \\
\mathrm{D} \\
\mathrm{E} \\
\mathrm{B}\end{array}$ & $\begin{array}{l}5658.28 \\
5664.69 \\
5669.44 \\
5678.93 \\
5680.37\end{array}$ & $\begin{array}{l}1 \\
1, b \\
1 \\
1 \\
1\end{array}$ & $\begin{array}{l}\mathrm{B} \\
\mathrm{D} \\
\mathrm{B} \\
\mathrm{E} \\
\mathrm{B}\end{array}$ & $\begin{array}{l}5741.25 \\
5742.80 \\
5748.31 \\
5750.54 \\
5751.73\end{array}$ & $\begin{array}{l}1 \\
1 \\
1, \text { b } 1 \\
1 \\
1\end{array}$ & $\begin{array}{l}\mathrm{C} \\
\mathrm{E} \\
\mathrm{C} \\
\mathrm{C} \\
\mathrm{C}\end{array}$ \\
\hline $\begin{array}{l}5602.93 \\
5604.11 \\
5607.09 \\
5608.96 \\
5610.90\end{array}$ & $\begin{array}{l}1 \\
1 \\
1 \\
1, h \\
2\end{array}$ & $\begin{array}{l}\text { B } \\
\mathbf{E} \\
\mathbf{E} \\
\mathbf{C} \\
\mathbf{B}\end{array}$ & $\begin{array}{l}5683.36 \\
5685.26 \\
5691.40 \\
5695.04 \\
5702.95\end{array}$ & $\begin{array}{l}1 \\
1 \\
1 \\
1, b, d ? \\
1\end{array}$ & $\begin{array}{l}\text { C } \\
\text { C } \\
\text { B } \\
\text { B } \\
\text { C }\end{array}$ & $\begin{array}{l}5757.35 \\
5758.25 \\
5760.35 \\
5761.87 \\
5763.57\end{array}$ & $\begin{array}{l}1 \\
2 \\
1 \\
1 \\
1\end{array}$ & $\begin{array}{l}\mathrm{C} \\
\mathrm{B} \\
\mathrm{E} \\
\mathrm{E} \\
\mathrm{C}\end{array}$ \\
\hline
\end{tabular}

${ }^{22}$ Kaiser. Akad. der Wiss. Sitz., Wien, 119, IIa, p. 595; т9ro. 
TABLE 7-Continued.

\begin{tabular}{|c|c|c|c|c|c|c|c|c|}
\hline$\lambda$ I. A. & Notes & $\begin{array}{c}\text { Probable } \\
\text { error }\end{array}$ & $\lambda$ I. A. & Notes & $\begin{array}{c}\text { Probable } \\
\text { error }\end{array}$ & $\lambda$ I. A. & Notes & $\begin{array}{c}\text { Probable } \\
\text { error }\end{array}$ \\
\hline $\begin{array}{l}5765.43 \\
5767.57 \\
5771.10 \\
5776.86 \\
5777.80\end{array}$ & $\begin{array}{l}1 \\
1, b \\
1 \\
1 \\
1\end{array}$ & $\begin{array}{l}\mathbf{C} \\
\mathbf{C} \\
\mathbf{C} \\
\mathbf{C} \\
\mathbf{C}\end{array}$ & $\begin{array}{l}5988.39 \\
5990.17 \\
5997.32 \\
5997.98 \\
5999.42\end{array}$ & $\begin{array}{l}1 \\
1 \\
2 \\
1 \\
1\end{array}$ & $\begin{array}{l}\mathbf{E} \\
\mathbf{C} \\
\mathbf{C} \\
\mathbf{B} \\
\mathbf{B}\end{array}$ & $\begin{array}{l}6254.16 \\
6261.41 \\
6268.63 \\
6270.58 \\
6279.59\end{array}$ & $\begin{array}{l}1 \\
1 \\
2, \mathrm{~b}, \mathrm{Cu} ? \\
\frac{1}{1}\end{array}$ & $\begin{array}{l}\text { B } \\
\mathbf{C} \\
\mathbf{C} \\
\mathbf{C} \\
\mathbf{C}\end{array}$ \\
\hline $\begin{array}{l}5779.66 \\
5780.59 \\
5787.76 \\
5788.60 \\
5796.49\end{array}$ & $\begin{array}{l}1 \\
2 \\
1 \\
1 \\
1\end{array}$ & $\begin{array}{l}\mathbf{E} \\
\mathbf{B} \\
\mathbf{E} \\
\mathbf{C} \\
\mathbf{C}\end{array}$ & $\begin{array}{l}6000.25 \\
6004.82 \\
6005.73 \\
6008.79 \\
6010.99\end{array}$ & $\begin{array}{l}1 \\
1 \\
1 \\
1 \\
1\end{array}$ & $\begin{array}{l}\mathbf{B} \\
\mathbf{C} \\
\mathbf{C} \\
\mathbf{B} \\
\mathbf{E}\end{array}$ & $\begin{array}{l}6280.14 \\
6291.97 \\
6293.37 \\
6298.55 \\
6301.28\end{array}$ & $\begin{array}{l}1 \\
2 \\
2 \\
2 \\
1\end{array}$ & $\begin{array}{l}\mathbf{C} \\
\mathbf{C} \\
\mathbf{C} \\
\mathbf{B} \\
\mathbf{E}\end{array}$ \\
\hline $\begin{array}{l}5798.57 \\
5800.80 \\
5802.13 \\
5803.76 \\
5805.33\end{array}$ & $\begin{array}{l}2 \\
1 \\
1 \\
1 \\
1\end{array}$ & $\begin{array}{l}\mathbf{C} \\
\mathbf{B} \\
\mathbf{C} \\
\mathbf{E} \\
\mathbf{C}\end{array}$ & $\begin{array}{l}6014.16 \\
6016.70 \\
6017.52 \\
6019.17 \\
6028.13\end{array}$ & $\begin{array}{l}1 \\
1 \\
2 \\
1 \\
1\end{array}$ & $\begin{array}{l}\mathbf{E} \\
\mathbf{C} \\
\mathbf{C} \\
\mathbf{C} \\
\mathbf{C}\end{array}$ & $\begin{array}{l}6304.61 \\
6306.85 \\
6321.28 \\
6322.37 \\
6330.74\end{array}$ & $\begin{array}{l}1 \\
1 \\
1 \\
1 \\
1\end{array}$ & $\begin{array}{l}\mathbf{B} \\
\mathbf{E} \\
\mathbf{E} \\
\mathbf{C} \\
\mathbf{E}\end{array}$ \\
\hline $\begin{array}{l}5811.28 \\
5812.30 \\
5813.89 \\
5814.54 \\
5826.11\end{array}$ & $\begin{array}{l}1 \\
1 \\
1 \\
1 \\
1 \\
1\end{array}$ & $\begin{array}{l}\mathbf{C} \\
\mathbf{E} \\
\mathbf{B} \\
\mathbf{C} \\
\mathbf{E}\end{array}$ & $\begin{array}{l}6039.65 \\
6050.57 \\
6051.74 \\
6053.07 \\
6056.95\end{array}$ & $\begin{array}{l}1 \\
1 \\
2 \\
1 \\
2, d ?\end{array}$ & $\begin{array}{l}\mathbf{C} \\
\mathbf{B} \\
\mathbf{B} \\
\mathbf{E} \\
\mathbf{B}\end{array}$ & $\begin{array}{l}6338.84 \\
6346.19 \\
6359.35 \\
6369.86 \\
6372.46\end{array}$ & $\begin{array}{l}1 \\
1 \\
2 \\
1 \\
2\end{array}$ & $\begin{array}{l}\mathbf{E} \\
\mathbf{E} \\
\mathbf{B} \\
\mathbf{C} \\
\mathbf{B}\end{array}$ \\
\hline $\begin{array}{l}5827.37 \\
5828.05 \\
5830.80 \\
5832.47 \\
5834.06\end{array}$ & $\begin{array}{l}1 \\
1 \\
1 \\
1 \\
1\end{array}$ & $\begin{array}{l}\mathbf{E} \\
\mathbf{E} \\
\mathbf{C} \\
\mathbf{C} \\
\mathbf{E}\end{array}$ & $\begin{array}{l}6059.73 \\
6062.29 \\
6065.91 \\
6067.22 \\
6074.84\end{array}$ & $\begin{array}{l}1 \\
1 \\
1 \\
1 \\
1\end{array}$ & $\begin{array}{l}\mathbf{C} \\
\mathbf{C} \\
\mathbf{C} \\
\mathbf{C} \\
\mathbf{E}\end{array}$ & $\begin{array}{l}6373.01 \\
6374.60 \\
6375.91 \\
6378.46 \\
6379.61\end{array}$ & $\begin{array}{l}1 \\
1, \mathrm{~b} v \\
1 \\
2 \\
2\end{array}$ & $\begin{array}{l}\mathbf{E} \\
\mathbf{C} \\
\mathbf{C} \\
\mathbf{B} \\
\mathbf{B}\end{array}$ \\
\hline $\begin{array}{l}5835.26 \\
5836.10 \\
5837.74 \\
5839.11 \\
5841.83\end{array}$ & $\begin{array}{l}1 \\
2 \\
2 \\
1 \\
1\end{array}$ & $\begin{array}{l}\mathbf{E} \\
\mathbf{C} \\
\mathbf{C} \\
\mathbf{E} \\
\mathbf{C}\end{array}$ & $\begin{array}{l}6077.28 \\
6079.55 \\
6080.40 \\
6083.74 \\
6084.91\end{array}$ & $\begin{array}{l}2 \\
1 \\
1 \\
1 \\
1\end{array}$ & $\begin{array}{l}\mathbf{B} \\
\mathbf{E} \\
\mathbf{B} \\
\mathbf{E} \\
\mathbf{E}\end{array}$ & $\begin{array}{l}6383.56 \\
6386.78 \\
6388.36 \\
6389.80 \\
6391.11\end{array}$ & $\begin{array}{l}1 \\
1 \\
1 \\
2 \\
1, h\end{array}$ & $\begin{array}{l}\mathbf{C} \\
\mathbf{E} \\
\mathbf{E} \\
\mathbf{B} \\
\mathbf{C}\end{array}$ \\
\hline $\begin{array}{l}5843.29 \\
5843.88 \\
5845.25 \\
5851.99 \\
5853.93\end{array}$ & $\begin{array}{l}1 \\
1 \\
2 \\
1 \\
1\end{array}$ & $\begin{array}{l}\mathbf{C} \\
\mathbf{C} \\
\mathbf{C} \\
\mathbf{C} \\
\mathbf{C}\end{array}$ & $\begin{array}{l}6087.35 \\
6089.16 \\
6099.85 \\
6101.76 \\
6110.86\end{array}$ & $\begin{array}{l}1 \\
1 \\
1 \\
1 \\
1\end{array}$ & $\begin{array}{l}\mathbf{B} \\
\mathbf{B} \\
\mathbf{B} \\
\mathbf{B} \\
\mathbf{C}\end{array}$ & $\begin{array}{l}6392.75 \\
6395.46 \\
6397.19 \\
6400.27 \\
6404.40\end{array}$ & $\begin{array}{l}2 \\
4 \\
2 \\
1 \\
1\end{array}$ & $\begin{array}{l}\mathbf{B} \\
\mathbf{B} \\
\mathbf{B} \\
\mathbf{B} \\
\mathbf{E}\end{array}$ \\
\hline $\begin{array}{l}5856.59 \\
5859.21 \\
5863.44 \\
5870.93 \\
5874.61\end{array}$ & $\begin{array}{l}1 \\
1 \\
1 \\
1 \\
1\end{array}$ & $\begin{array}{l}\mathbf{C} \\
\mathbf{E} \\
\mathbf{C} \\
\mathbf{B} \\
\mathbf{E}\end{array}$ & $\begin{array}{l}6112.07 \\
6127.64 \\
6129.76 \\
6131.73 \\
6132.65\end{array}$ & $\begin{array}{l}1 \\
1 \\
2 \\
1 \\
2\end{array}$ & $\begin{array}{l}\mathbf{B} \\
\mathbf{C} \\
\mathbf{C} \\
\mathbf{E} \\
\mathbf{B}\end{array}$ & $\begin{array}{l}6411.65 \\
6424.89 \\
6427.39 \\
6428.83 \\
6430.95\end{array}$ & $\begin{array}{l}1 \\
1 \\
1 \\
1 \\
1\end{array}$ & $\begin{array}{l}\mathbf{B} \\
\mathbf{B} \\
\mathbf{C} \\
\mathbf{B} \\
\mathbf{C}\end{array}$ \\
\hline $\begin{array}{l}5886.98 \\
5892.61 \\
5893.75 \\
5898.80 \\
5902.54\end{array}$ & $\begin{array}{l}1 \\
1 \\
1 \\
1 \\
1\end{array}$ & $\begin{array}{l}\mathbf{C} \\
\mathbf{C} \\
\mathbf{C} \\
\mathbf{C} \\
\mathbf{C}\end{array}$ & $\begin{array}{l}6138.57 \\
6140.37 \\
6141.80 \\
6152.26 \\
6153.72\end{array}$ & $\begin{array}{l}1 \\
1 \\
1 \\
1 \\
1\end{array}$ & $\begin{array}{l}\mathbf{B} \\
\mathbf{B} \\
\mathbf{E} \\
\mathbf{B} \\
\mathbf{C}\end{array}$ & $\begin{array}{l}6449.20 \\
6464.99 \\
6470.58 \\
6477.90 \\
6479.91\end{array}$ & $\begin{array}{l}4 \\
3 \\
1 \\
1 \\
1\end{array}$ & $\begin{array}{l}\mathbf{B} \\
\mathbf{B} \\
\mathbf{B} \\
\mathbf{C} \\
\mathbf{C}\end{array}$ \\
\hline $\begin{array}{l}5915.38 \\
5925.39 \\
5929.35 \\
5930.33 \\
5932.54\end{array}$ & $\begin{array}{l}4 \\
1 \\
1 \\
1 \\
1\end{array}$ & $\begin{array}{l}\mathbf{C} \\
\mathbf{C} \\
\mathbf{C} \\
\mathbf{E} \\
\mathbf{E}\end{array}$ & $\begin{array}{l}6157.59 \\
6164.57 \\
6165.68 \\
6169.04 \\
6171.91\end{array}$ & $\begin{array}{l}1 \\
2 \\
1 \\
1 \\
2\end{array}$ & $\begin{array}{l}\mathbf{E} \\
\mathbf{B} \\
\mathbf{E} \\
\mathbf{E} \\
\mathbf{B}\end{array}$ & $\begin{array}{l}6481.74 \\
6487.05 \\
6488.34 \\
6495.35 \\
6503.59\end{array}$ & $\begin{array}{l}1 \\
1 \\
1 \\
1 \\
2\end{array}$ & $\begin{array}{l}\mathbf{B} \\
\underset{\mathbf{E}}{\mathbf{B}} \\
\mathbf{C} \\
\mathbf{B}\end{array}$ \\
\hline $\begin{array}{l}5933.85 \\
5935.38 \\
5948.53 \\
5949.85 \\
5952.06\end{array}$ & $\begin{array}{l}1 \\
1, b, h \\
1 \\
1 \\
1\end{array}$ & $\begin{array}{l}\text { C } \\
\text { C } \\
\text { B } \\
\text { B } \\
\text { B }\end{array}$ & $\begin{array}{l}6172.86 \\
6174.42 \\
6175.44 \\
6181.36 \\
6196.46\end{array}$ & $\begin{array}{l}1 \\
1 \\
2 \\
1 \\
1\end{array}$ & $\begin{array}{l}\mathbf{B} \\
\mathbf{C} \\
\mathbf{C} \\
\mathbf{E} \\
\mathbf{E}\end{array}$ & $\begin{array}{l}6506.32 \\
6510.85 \\
6511.21 \\
6515.49 \\
6517.73\end{array}$ & $\begin{array}{l}1 \\
1 \\
1, b \\
1 \\
1\end{array}$ & $\begin{array}{l}\mathbf{B} \\
\mathbf{B} \\
\mathbf{C} \\
\mathbf{E} \\
\mathbf{E}\end{array}$ \\
\hline $\begin{array}{l}5955.03 \\
5956.89 \\
5963.91 \\
5971.56 \\
5972.84\end{array}$ & $\begin{array}{l}1 \\
1 \\
1 \\
2 \\
1, \mathrm{~b}\end{array}$ & $\begin{array}{l}\mathbf{E} \\
\mathbf{C} \\
\mathbf{E} \\
\mathbf{B} \\
\mathbf{B}\end{array}$ & $\begin{array}{l}6198.40 \\
6202.03 \\
6203.30 \\
6215.37 \\
6222.12\end{array}$ & $\begin{array}{l}1 \\
1 \\
1 \\
2 \\
1\end{array}$ & $\begin{array}{l}\mathbf{E} \\
\mathbf{E} \\
\mathbf{E} \\
\mathbf{B} \\
\mathbf{C}\end{array}$ & $\begin{array}{l}6518.93 \\
6520.95 \\
6526.05 \\
6527.11 \\
6534.66\end{array}$ & $\begin{array}{l}2 \\
1 \\
1 \\
1 \\
1\end{array}$ & $\begin{array}{l}\mathbf{B} \\
\mathbf{C} \\
\mathbf{B} \\
\mathbf{B} \\
\mathbf{E}\end{array}$ \\
\hline $\begin{array}{l}5974.79 \\
5976.34 \\
5982.72 \\
5984.65 \\
5986.15\end{array}$ & $\begin{array}{l}1, b, v \\
3 \\
1 \\
1 \\
2\end{array}$ & $\begin{array}{l}\mathbf{C} \\
\mathbf{B} \\
\mathbf{E} \\
\mathbf{E} \\
\mathbf{B}\end{array}$ & $\begin{array}{l}6232.48 \\
6234.31 \\
6246.52 \\
6248.07 \\
6251.76\end{array}$ & $\begin{array}{l}1, \mathbf{b} \\
1 \\
1 \\
1 \\
1\end{array}$ & $\begin{array}{l}\mathbf{B} \\
\mathbf{B} \\
\mathbf{B} \\
\mathbf{E} \\
\mathbf{B}\end{array}$ & $\begin{array}{l}6535.41 \\
6536.58 \\
6537.54 \\
6542.96 \\
6544.01\end{array}$ & $\begin{array}{l}1 \\
1 \\
1 \\
2 \\
1\end{array}$ & $\begin{array}{l}\mathbf{B} \\
\mathbf{E} \\
\mathbf{E} \\
\mathbf{B} \\
\mathbf{E}\end{array}$ \\
\hline
\end{tabular}


TABLE 7-Continued

\begin{tabular}{l|l|l||l|l|l||l|l|l}
\hline$\lambda$ I. A. & Notes & $\begin{array}{c}\text { Probable } \\
\text { error }\end{array}$ & $\lambda$ I. A. & Notes & $\begin{array}{c}\text { Probable } \\
\text { error }\end{array}$ & $\lambda$ I. A. & Notes & Probable \\
error \\
\hline
\end{tabular}


TABLE 7-Continued

\begin{tabular}{|c|c|c|c|c|c|c|c|c|}
\hline$\lambda$ I. A. & Notes & $\begin{array}{c}\text { Probable } \\
\text { error }\end{array}$ & $\lambda$ I. A. & Notes & $\begin{array}{c}\text { Probable } \\
\text { error }\end{array}$ & $\lambda$ I. A. & Notes & $\begin{array}{c}\text { Probable } \\
\text { error }\end{array}$ \\
\hline $\begin{array}{l}7119.46 \\
7121.83 \\
7126.19 \\
7128.88 \\
7130.06\end{array}$ & $\begin{array}{l}1 \\
1 \\
2 \\
4 \\
1\end{array}$ & $\begin{array}{l}\mathrm{E} \\
\mathrm{E} \\
\mathrm{C} \\
\mathrm{B} \\
\mathrm{B}\end{array}$ & $\begin{array}{l}7438.20 \\
7443.07 \\
7443.90 \\
7447.85 \\
7452.48\end{array}$ & $\begin{array}{l}2 \\
2 \\
1 \\
1 \\
1\end{array}$ & $\begin{array}{l}\mathbf{B} \\
\mathbf{B} \\
\mathbf{B} \\
\mathrm{B} \\
\mathbf{C}\end{array}$ & $\begin{array}{l}7669.69 \\
7689.91 \\
7691.89 \\
7693.59 \\
7696.88\end{array}$ & $\begin{array}{l}2 \\
1 \\
1 \\
1 \\
1\end{array}$ & $\begin{array}{l}\mathrm{C} \\
\mathrm{E} \\
\mathrm{C} \\
\mathrm{C} \\
\mathrm{E} \\
\mathrm{B}\end{array}$ \\
\hline $\begin{array}{l}7135.98 \\
7137.95 \\
7139.92 \\
7141.18 \\
7142.24\end{array}$ & $\begin{array}{l}1 \\
1 \\
1 \\
1 \\
1\end{array}$ & $\begin{array}{l}\mathrm{E} \\
\mathrm{E} \\
\mathrm{E} \\
\mathrm{E} \\
\mathrm{E}\end{array}$ & $\begin{array}{l}7453.93 \\
7457.76 \\
7467.06 \\
740.87 \\
7476.08\end{array}$ & $\begin{array}{l}2 \\
1 \\
1 \\
1 \\
1\end{array}$ & $\begin{array}{l}\mathbf{B} \\
\mathbf{C} \\
\mathbf{C} \\
\mathbf{E} \\
\mathbf{E}\end{array}$ & $\begin{array}{l}7707.59 \\
7718.87 \\
7744.30 \\
7747.09 \\
7748.14\end{array}$ & $\begin{array}{l}1 \\
1 \\
1 \\
1 \\
1\end{array}$ & $\begin{array}{l}\mathbf{E} \\
\mathbf{E} \\
\mathbf{E} \\
\mathbf{C} \\
\mathbf{B}\end{array}$ \\
\hline $\begin{array}{l}7144.06 \\
7147.89 \\
7152.08 \\
7155.76 \\
7164.93\end{array}$ & $\begin{array}{l}1 \\
2 \\
1 \\
1 \\
2\end{array}$ & $\begin{array}{l}\mathbf{E} \\
\mathbf{B} \\
\mathbf{E} \\
\mathbf{E} \\
\mathbf{B}\end{array}$ & $\begin{array}{l}7477.40 \\
7480.49 \\
7483.48 \\
7484.88 \\
7489.17\end{array}$ & $\begin{array}{l}1 \\
1 \\
1 \\
1 \\
1\end{array}$ & $\begin{array}{l}\mathrm{E} \\
\mathrm{C} \\
\mathrm{D} \\
\mathrm{D} \\
\mathrm{C}\end{array}$ & $\begin{array}{l}7754.16 \\
7759.85 \\
7761.82 \\
7763.77 \\
7766.57\end{array}$ & $\begin{array}{l}1 \\
1 \\
2 \\
1 \\
1\end{array}$ & $\begin{array}{l}\mathbf{B} \\
\mathbf{B} \\
\mathbf{B} \\
\mathbf{E} \\
\mathbf{E}\end{array}$ \\
\hline $\begin{array}{l}7168.02 \\
7170.68 \\
7172.16 \\
7175.92 \\
7179.27\end{array}$ & $\begin{array}{l}1 \\
1 \\
1 \\
1 \\
2, \mathrm{~b}\end{array}$ & $\begin{array}{l}\mathrm{E} \\
\mathrm{E} \\
\mathrm{C} \\
\mathrm{E} \\
\mathrm{C}\end{array}$ & $\begin{array}{l}7494.21 \\
7497.31 \\
7499.50 \\
7502.24 \\
7504.95\end{array}$ & $\begin{array}{l}\text { 1, b } \\
1 \\
1 \\
1 \\
1\end{array}$ & $\begin{array}{l}\mathbf{B} \\
\mathbf{E} \\
\mathbf{E} \\
\mathbf{C} \\
\mathbf{C}\end{array}$ & $\begin{array}{l}7768.93 \\
7776.94 \\
7780.49 \\
7782.38 \\
7784.11\end{array}$ & $\begin{array}{l}1 \\
1, \\
1 \\
1 \\
5\end{array}$ & $\begin{array}{l}\mathbf{E} \\
\mathbf{C} \\
\mathrm{B} \\
\mathrm{D} \\
\mathrm{B}\end{array}$ \\
\hline $\begin{array}{l}7183.46 \\
7187.51 \\
7189.35 \\
7193.10 \\
7194.64\end{array}$ & $\begin{array}{l}2 \\
1 \\
1 \\
1 \\
1\end{array}$ & $\begin{array}{l}\mathrm{D} \\
\mathrm{E} \\
\mathrm{E} \\
\mathrm{D} \\
\mathrm{C}\end{array}$ & $\begin{array}{l}7506.94 \\
7510.04 \\
7512.58 \\
7514.37 \\
7517.37\end{array}$ & $\begin{array}{l}\text { 1, b } \\
1 \\
1 \\
1 \\
1\end{array}$ & $\begin{array}{l}\mathrm{B} \\
\mathrm{B} \\
\mathrm{E} \\
\mathrm{E} \\
\mathrm{E} \\
\mathrm{B}\end{array}$ & $\begin{array}{l}7791.24 \\
7802.32 \\
7808.36 \\
7813.42 \\
7816.27\end{array}$ & $\begin{array}{l}1 \\
1 \\
1 \\
1 \\
1\end{array}$ & $\begin{array}{l}\mathrm{E} \\
\mathrm{C} \\
\mathrm{C} \\
\mathrm{E} \\
\mathrm{B}\end{array}$ \\
\hline $\begin{array}{l}7196.57 \\
7205.39 \\
7207.46 \\
7209.24 \\
7210.29\end{array}$ & $\begin{array}{l}1 \\
1 \\
1 \\
1 \\
1\end{array}$ & $\begin{array}{l}\mathrm{C} \\
\mathrm{C} \\
\mathrm{E} \\
\mathrm{C} \\
\mathrm{C}\end{array}$ & $\begin{array}{l}7528.68 \\
7531.68 \\
7533.91 \\
7550.15 \\
7552.13\end{array}$ & $\begin{array}{l}2 \\
1 \\
5 \\
2 \\
1\end{array}$ & $\begin{array}{l}\mathbf{B} \\
\underset{\mathbf{E}}{\mathbf{B}} \\
\stackrel{\mathbf{B}}{\mathrm{B}} \\
\mathbf{E}\end{array}$ & $\begin{array}{l}7821.82 \\
7823.79 \\
7832.02 \\
7835.57 \\
7837.66\end{array}$ & $\begin{array}{l}1 \\
1 \\
1 \\
1 \\
1\end{array}$ & $\begin{array}{l}\mathrm{C} \\
\mathrm{C} \\
\mathbf{C} \\
\mathbf{C} \\
\mathbf{B}\end{array}$ \\
\hline $\begin{array}{l}7212.08 \\
7218.01 \\
7228.08 \\
7230.86 \\
7251.94\end{array}$ & $\begin{array}{l}\frac{1}{2} \\
1, \mathrm{~b} \\
1 \\
1\end{array}$ & $\begin{array}{l}\mathbf{C} \\
\mathbf{C} \\
\mathbf{C} \\
\mathrm{E} \\
\mathrm{E}\end{array}$ & $\begin{array}{l}7558.31 \\
7560.69 \\
7562.44 \\
7566.02 \\
7570.72\end{array}$ & $\begin{array}{l}1 \\
1 \\
1 \\
2 \\
1\end{array}$ & $\begin{array}{l}\mathbf{E} \\
\mathbf{E} \\
\mathbf{E} \\
\mathbf{B} \\
\mathbf{E}\end{array}$ & $\begin{array}{l}7844.75 \\
7868.78 \\
7875.34 \\
7881.91 \\
7895.92\end{array}$ & $\begin{array}{l}1 \\
2 \\
2 \\
4 \\
1\end{array}$ & $\begin{array}{l}\text { B } \\
\text { B } \\
\text { B } \\
\text { B } \\
\text { C }\end{array}$ \\
\hline $\begin{array}{l}7254.46 \\
7256.57 \\
7258.99 \\
7273.71 \\
7279.79\end{array}$ & $\begin{array}{l}2 \\
1 \\
1, b \\
1 \\
1\end{array}$ & $\begin{array}{l}\mathbf{B} \\
\mathbf{C} \\
\mathbf{C} \\
\mathrm{C} \\
\mathrm{E}\end{array}$ & $\begin{array}{l}7572.37 \\
7573.14 \\
7575.80 \\
7580.79 \\
7582.21\end{array}$ & $\begin{array}{l}1, b \\
1 \\
1 \\
1 \\
1\end{array}$ & $\begin{array}{l}\mathbf{B} \\
\mathbf{C} \\
\mathbf{B} \\
\mathbf{B} \\
\mathbf{E}\end{array}$ & $\begin{array}{l}7900.40 \\
7904.22 \\
7907.91 \\
7918.75 \\
7922.04\end{array}$ & $\begin{array}{l}1 \\
1 \\
1 \\
1 \\
1\end{array}$ & $\begin{array}{l}\mathrm{B} \\
\mathrm{B} \\
\mathrm{B} \\
\mathrm{B} \\
\mathrm{C}\end{array}$ \\
\hline $\begin{array}{l}7282.37 \\
7293.97 \\
7299.42 \\
7312.45 \\
7314.46\end{array}$ & $\begin{array}{l}1 \\
1 \\
1 \\
1 \\
1\end{array}$ & $\begin{array}{l}\mathrm{E} \\
\mathrm{E} \\
\mathrm{E} \\
\mathrm{E} \\
\mathbf{E}\end{array}$ & $\begin{array}{l}7587.68 \\
7590.45 \\
7595.06 \\
7597.46 \\
7600.27\end{array}$ & $\begin{array}{l}1 \\
1, \mathrm{~b} \\
1, \mathrm{~b} \\
1\end{array}$ & $\begin{array}{l}\text { B } \\
\text { B } \\
\text { B } \\
\text { D } \\
\text { C }\end{array}$ & $\begin{array}{l}7959.83 \\
7963.95 \\
7967.09 \\
7970.44 \\
7975.15\end{array}$ & $\begin{array}{l}1 \\
1 \\
1 \\
3 \\
2, \mathrm{~b}, \mathrm{~h}\end{array}$ & $\begin{array}{l}\mathrm{C} \\
\mathrm{B} \\
\mathrm{E} \\
\mathrm{B} \\
\mathrm{B}\end{array}$ \\
\hline $\begin{array}{l}7315.87 \\
7331.86 \\
733.56 \\
7335.80 \\
7341.66\end{array}$ & $\begin{array}{l}\frac{1}{2, b, v} \\
1 \\
\frac{1}{2, \mathrm{~b}}\end{array}$ & $\begin{array}{l}\mathrm{E} \\
\mathrm{C} \\
\mathrm{C} \\
\mathrm{E} \\
\mathrm{C}\end{array}$ & $\begin{array}{l}7605.90 \\
7609.15 \\
7610.77 \\
7615.72 \\
7619.34\end{array}$ & $\begin{array}{l}1 \\
2 \\
1 \\
1 \\
3\end{array}$ & $\begin{array}{l}\mathrm{C} \\
\mathrm{B} \\
\mathrm{E} \\
\mathrm{E} \\
\mathrm{E}\end{array}$ & $\begin{array}{l}7976.86 \\
7978.62 \\
7989.13 \\
7991.32 \\
7995.44\end{array}$ & $\begin{array}{l}1 \\
1 \\
1 \\
1 \\
1\end{array}$ & $\begin{array}{l}\text { B } \\
\text { C } \\
\text { C } \\
\text { D }\end{array}$ \\
\hline $\begin{array}{l}7361.07 \\
7368.65 \\
7370.21 \\
7372.09 \\
7372.68\end{array}$ & $\begin{array}{l}1 \\
1 \\
1 \\
2 \\
1\end{array}$ & $\begin{array}{l}\mathrm{E} \\
\mathrm{D} \\
\mathrm{D} \\
\mathrm{C} \\
\mathrm{E}\end{array}$ & $\begin{array}{l}7621.86 \\
7626.15 \\
7627.96 \\
7629.34 \\
7630.44\end{array}$ & $\begin{array}{l}1 \\
1 \\
1 \\
1 \\
1\end{array}$ & $\begin{array}{l}\text { B } \\
\text { E } \\
\text { D } \\
\text { D } \\
\mathbf{E}\end{array}$ & $\begin{array}{l}7998.52 \\
8002.91 \\
8019.27 \\
8034.69 \\
8035.99\end{array}$ & $\begin{array}{l}1 \\
1, \\
1 \\
1 \\
1\end{array}$ & $\begin{array}{l}\text { B } \\
\text { D } \\
\mathbf{E} \\
\text { B } \\
\mathbf{E}\end{array}$ \\
\hline $\begin{array}{l}7375.40 \\
7377.29 \\
7379.63 \\
7390.89 \\
7392.11\end{array}$ & $\begin{array}{l}1 \\
1 \\
2 \\
1 \\
1\end{array}$ & $\begin{array}{l}\mathrm{D} \\
\mathrm{C} \\
\mathrm{C} \\
\mathrm{E} \\
\mathrm{C}\end{array}$ & $\begin{array}{l}7631.72 \\
7632.70 \\
7634.78 \\
7639.56 \\
7641.24\end{array}$ & $\begin{array}{l}3 \\
1 \\
2 \\
2 \\
1\end{array}$ & $\begin{array}{l}\mathrm{B} \\
\mathrm{E} \\
\mathrm{B} \\
\mathrm{B} \\
\mathrm{B} \\
\mathrm{E}\end{array}$ & $\begin{array}{l}8055.56 \\
8060.34 \\
8065.68 \\
8067.93 \\
8074.03\end{array}$ & $\begin{array}{l}1 \\
1 \\
1, \\
1 \\
1\end{array}$ & $\begin{array}{l}\mathrm{C} \\
\mathrm{E} \\
\mathrm{B} \\
\mathrm{E} \\
\mathrm{C}\end{array}$ \\
\hline $\begin{array}{l}7396.97 \\
746.36 \\
7419.57 \\
77425.49 \\
7432.21\end{array}$ & $\begin{array}{l}2 \\
1 \\
1 \\
3 \\
1\end{array}$ & $\begin{array}{l}\text { B } \\
\text { C } \\
\text { E } \\
\text { B } \\
\text { C }\end{array}$ & $\begin{array}{l}7643.67 \\
7648.66 \\
7653.55 \\
7658.76 \\
7668.59\end{array}$ & $\begin{array}{l}1 \\
1 \\
1 \\
1 \\
2, \mathrm{~h}\end{array}$ & $\begin{array}{l}\mathrm{E} \\
\mathrm{E} \\
\mathrm{C} \\
\mathrm{E} \\
\mathrm{E}\end{array}$ & $\begin{array}{l}8097.52 \\
8174.29 \\
8175.77 \\
8188.18 \\
8262.04\end{array}$ & $\begin{array}{l}1 \\
2 \\
1 \\
1 \\
2\end{array}$ & $\begin{array}{l}\mathrm{E} \\
\mathrm{B} \\
\mathrm{E} \\
\mathrm{E} \\
\mathrm{B} \\
\mathrm{B}\end{array}$ \\
\hline
\end{tabular}


TABLE 7-Continued

\begin{tabular}{|c|c|c|c|c|c|c|c|c|}
\hline$\lambda$ I. A. & Notes & $\begin{array}{l}\text { Probable } \\
\text { error }\end{array}$ & $\lambda \mathbf{I} . \mathbf{A}$. & Notes & $\begin{array}{c}\text { Probable } \\
\text { error }\end{array}$ & $\lambda I_{0} A_{0}$ & Notes & $\begin{array}{c}\text { Probable } \\
\text { error }\end{array}$ \\
\hline $\begin{array}{l}8318.36 \\
8327.79 \\
8329.83 \\
8337.49 \\
8346.74\end{array}$ & $\begin{array}{l}1 \\
1 \\
1 \\
1 \\
1\end{array}$ & $\begin{array}{l}\mathbf{B} \\
\stackrel{\mathbf{E}}{\mathbf{E}} \\
\mathbf{B} \\
\mathbf{C}\end{array}$ & $\begin{array}{l}8557.36 \\
8566.92 \\
8567.72 \\
8570.60 \\
8574.55\end{array}$ & $\begin{array}{l}1 \\
1 \\
1 \\
2 \\
1\end{array}$ & $\begin{array}{l}\mathrm{C} \\
\mathrm{E} \\
\mathrm{E} \\
\mathrm{C} \\
\mathrm{C}\end{array}$ & $\begin{array}{l}9023.38 \\
9093.61 \\
9136.80 \\
9139.21\end{array}$ & $\begin{array}{l}1, \mathrm{~h} \\
1 \\
0 \\
1\end{array}$ & $\begin{array}{l}\mathbf{B} \\
\mathbf{B} \\
\mathbf{E} \\
\mathbf{E}\end{array}$ \\
\hline $\begin{array}{l}8357.07 \\
8331.92 \\
8387.25 \\
8389.12 \\
8390.95\end{array}$ & $\begin{array}{l}1 \\
2 \\
2 \\
2 \\
1\end{array}$ & $\begin{array}{l}\mathbf{E} \\
\mathbf{B} \\
\mathbf{C} \\
\mathbf{C} \\
\mathbf{E}\end{array}$ & $\begin{array}{l}8607.92 \\
8612.82 \\
8618.77 \\
8637.48 \\
8641.09\end{array}$ & $\begin{array}{l}3 \\
1 \\
1 \\
1 \\
1\end{array}$ & $\begin{array}{l}\mathrm{B} \\
\mathrm{E} \\
\mathrm{E} \\
\mathrm{E} \\
\mathrm{E}\end{array}$ & $\begin{array}{l}9140.97 \\
9143.20 \\
9276.24 \\
9530.38\end{array}$ & $\begin{array}{l}1 \\
1 \\
1 \\
1, \mathbf{h}\end{array}$ & $\begin{array}{l}\mathbf{E} \\
\mathbf{E} \\
\mathbf{E} \\
\mathbf{E}\end{array}$ \\
\hline $\begin{array}{l}8396.82 \\
8399.26 \\
8426.70 \\
8441.25 \\
8445.35\end{array}$ & $\begin{array}{l}1 \\
1 \\
1 \\
1 \\
2\end{array}$ & $\begin{array}{l}\mathrm{E} \\
\mathrm{E} \\
\mathrm{E} \\
\mathrm{E} \\
\mathrm{B}\end{array}$ & $\begin{array}{l}8652.74 \\
8659.50 \\
8691.28 \\
8710.73 \\
8728.65\end{array}$ & $\begin{array}{l}1 \\
1 \\
2 \\
2, \mathrm{~h} \\
1\end{array}$ & $\begin{array}{l}\mathrm{E} \\
\mathrm{E} \\
\mathrm{B} \\
\mathrm{C} \\
\mathrm{E}\end{array}$ & & & \\
\hline $\begin{array}{l}8450.03 \\
8446.09 \\
8504.70 \\
8531.10 \\
8540.13\end{array}$ & $\begin{array}{l}2 \\
2 \\
2 \\
1 \\
2\end{array}$ & $\begin{array}{l}\mathbf{B} \\
\mathbf{C} \\
\mathbf{C} \\
\mathbf{E} \\
\mathbf{C}\end{array}$ & $\begin{array}{l}8753.64 \\
875.83 \\
8860.62 \\
8951.92 \\
9020.89\end{array}$ & $\begin{array}{l}1 \\
2 \\
1 \\
1 \\
1, \mathrm{~h}\end{array}$ & $\begin{array}{l}\mathrm{C} \\
\mathrm{D} \\
\mathrm{E} \\
\mathrm{C} \\
\mathrm{B}\end{array}$ & & & \\
\hline
\end{tabular}

TABLE 8.-Faint Lines Common to the Spectra of Two or More Elements

\begin{tabular}{|c|c|c|c|c|c|}
\hline$\lambda$ I. A. & $\mathbf{i}$ & Notes & $\lambda$ I. A. & 1 & Notes \\
\hline $\begin{array}{l}5536.49 \\
5554.97 \\
5752.85 \\
5756.81 \\
5995.77\end{array}$ & $\begin{array}{l}1 \\
1,0 ? \\
1 \\
1 \\
1\end{array}$ & $\begin{array}{l}\mathrm{Co}, \mathrm{Mn} \\
\mathrm{Mn}, \mathrm{Cu} \\
\mathrm{Co}, \mathrm{Ti}, \mathrm{V} \\
\mathrm{Fe}, \mathrm{Ti} \\
\mathrm{Co}, \mathrm{Ti}\end{array}$ & $\begin{array}{l}7135.62 \\
7139.71 \\
7221.22 \\
7443.49 \\
7483.43\end{array}$ & $\begin{array}{l}2 \\
2 \\
2 \\
2 \\
2\end{array}$ & $\begin{array}{l}\text { Mn, V, Ti } \\
M n, V \\
\text { Fe, Mn } \\
\text { Co, Mn } \\
\text { Fe, Mn, U }\end{array}$ \\
\hline $\begin{array}{l}6062.90 \\
6078.44 \\
6114.30 \\
6374.51 \\
6529.08\end{array}$ & $\begin{array}{l}1, \mathrm{Nh} ? \\
3 \\
1 \\
1 \\
1\end{array}$ & $\begin{array}{l}\mathrm{Fe}, \mathrm{Mn}, \mathrm{Si} \\
\mathrm{Fe}, \mathrm{Mn} \\
\mathrm{Co}, \mathrm{Mn} \\
\mathrm{Co}, \mathrm{V} \\
\mathrm{Fe}, \mathrm{Cr}\end{array}$ & $\begin{array}{l}7552.22 \\
7624.79 \\
7735.95 \\
8028.32 \\
8032.45\end{array}$ & $\begin{array}{l}2 \\
2 \\
1 \\
2 \\
1\end{array}$ & $\begin{array}{l}\mathrm{Ni}, \mathrm{Mn}, \mathrm{U} \\
\mathrm{Ni}, \mathrm{V} \\
\mathrm{Ni}, \mathrm{Mn} \\
\mathrm{Fe}, \mathrm{Ni}, \mathrm{Cr}, \mathrm{Mn} \\
\mathrm{Co}, \mathrm{Mn}\end{array}$ \\
\hline \multirow[t]{2}{*}{$\begin{array}{l}6558.03 \\
6777.82 \\
6789.25 \\
6906.36 \\
6933.62\end{array}$} & $\begin{array}{l}2 \\
1 \\
3 \\
1 \\
2, Y t ?\end{array}$ & $\begin{array}{l}M n, V \\
M n, C r \\
C o, C r \\
C o, M n \\
\text { Fe, Mn }\end{array}$ & $\begin{array}{l}8179.05 \\
8481.76 \\
8514.02 \\
8569.75 \\
8734.66\end{array}$ & $\begin{array}{l}1 \\
1 \\
2, \mathrm{Ba} ? \\
2 \\
1\end{array}$ & $\begin{array}{l}\text { Fe, Mn } \\
\text { Mn, V } \\
\text { Fe, Mn } \\
\text { Co, Ti } \\
\text { Mn, Ti }\end{array}$ \\
\hline & & & $\begin{array}{l}8945.15 \\
9012.04\end{array}$ & $\begin{array}{l}1 \\
1\end{array}$ & $\begin{array}{l}\mathrm{Fe}, \mathrm{Ti} \\
\mathrm{Fe}, \mathbf{N i}, \mathbf{T i}\end{array}$ \\
\hline
\end{tabular}

\section{FAINT LINES COMMON TO SEVERAL SPECTRA}

Table 8 contains about 30 faint lines which appear with equal intensity in the spectra of two or more elements. Undoubtedly these lines owe their origin to some common impurities which have not as yet been identified. In a previous paper ${ }^{23}$ attention has been called to the presence of two groups of lines belonging to oxygen, which appear in the arc spectra of metals. These lines have been measured repeatedly during the work described in this paper, and, in addition, other lines agreeing in wave length with lines in the series spectrum of oxygen have been 
observed. Thus, the line 5554.97 has been measured in the spectra of manganese and copper, 6456.06 in that of titanium, and 9263.92 in the spectrum of chromium. This last line was not given by Randall and Barker ${ }^{24}$ in the list of infra-red chromium lines observed by them with the bolometer.

\section{SUMMARY}

The concave-grating spectrograph of the Bureau of Standards was used in photographing the yellow, red, and infra-red arc spectra of titanium, vanadium, chromium, manganese, molybdenum, tungsten, and uranium. The photographs were made on plates sensitized to the red and infra-red rays with pinacyanol and dicyanin. American-made dyes were used for many of the plates and were as effective in their sensitizing power as their predecessors. More than 2600 wave lengths were derived from the measurement of the plates, many of them being the wave lengths of heads of bands which are prominent in the spectra of titanium, vanadium, and chromium. Impurity lines have been eliminated from the tables, so far as known, those not identified being brought together in a separate table. Lines whose wave lengths agree with those in the series spectrum of oxygen have been observed in the spectra tabulated above. Frequency differences which are suspected of being constant have been found in each of the spectra here presented.

WASHINGTON, November 8, I9I9. 



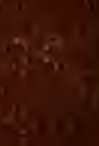

